\title{
Thermal Evaluation of Vacuum Insulation Panels within Residential Building Envelopes
}

\author{
by \\ Brock Conley, B.Eng., Mechanical Engineering \\ Carleton University
}

A thesis submitted to the Faculty of Graduate and Postdoctoral Affairs in partial fulfillment of the requirements for the degree of

Master of Applied Science

in

Mechanical Engineering

Carleton University

Ottawa, Ontario

(C) 2017, Brock Conley 


\begin{abstract}
In Canada, heating and cooling in buildings accounts for $16.5 \%$ of the total secondary end energy use in the residential sector [1]. Improvements in the building enclosure and overall thermal resistance can reduce the energy required to maintain the interior conditions through additional insulation or high performance insulations. The thesis analyzes the effects of vacuum insulation panels (VIPs) installed within a residential building envelope. The VIPs were evaluated using a guarded hot-box operating at steady-state conditions. Several paneling and tiling techniques were performed and evaluated in the guarded hot-box to quantify the change in performance between the panel center and edges. Infrared thermal images were captured to validate the temperature gradients expected at the edge of VIPs. The edge and center thermal performance of the VIP was experimentally evaluated, and showed a 36\% decay in performance at the edge, when compared to the center, indicating that the material acts non-homogenous within the building envelope. An assembled composite panel of extruded polystyrene (XPS) and VIPs was examined as a method to address the fragility concerns of VIPs, while improving the thermal resistance of the building envelope. In total, five different walls designs were evaluated by the varying VIP sizes and fastening strips thicknesses. THERM two-dimensional heat transfer software was used to create a single representative cross-section of the various composite wall assemblies by using two fictitious stud sizes and the material's wall area coverage. The modelling method was compared to experimental results and conventional modelling methods to determine the new model's effectiveness. The results indicate that the VIP-XPS panels offer improved performance compared to conventional insulation systems and minimize the risk of puncture. They also show that a single profile simulation can provide reliable results of a wall assembly containing multiple nonhomogenous layers.
\end{abstract}




\section{Acknowledgements}

I would like to thank my colleagues Chris, Nina, Kevin, Sarah, Dave, Dan, Jayson, Pat, Alex, Adam, and Dylan within the Solar Energy Systems Laboratory who have provided me friendship, help, and made it an enjoyable environment to work throughout the duration of my degree. They allowed for a safe, friendly, and productive workspace that allowed me to conduct experiments and successfully troubleshoot issues as they arose.

I would like to thank my many athletic endeavors that included the Carleton University Lacrosse Club, Leeds Lightning, and Snowflakes for providing me an outlet whenever we played. Altogether, we achieved one championship with a million memories.

I would also like to express my sincere appreciation and gratitude towards my supervisor, Dr. Cynthia Cruickshank who mentored and guided me through the difficulties associated with research work. Her positive attitude and desire to push me forward has allowed me to reach achievements and opportunities that I could not have dreamt.

Finally yet importantly, I thank my parents Paul and Alison, my brothers John, Greg and Curtis, and finally, friends for their support and encouragement from beginning to end of this degree. I could not imagine being surrounded by better group of people. Thank you and love you. 


\section{Table of Contents}

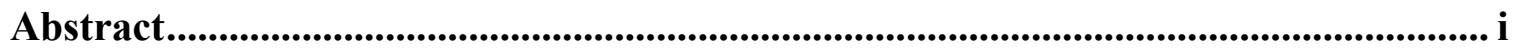

Acknowledgements .............................................................................................................. ii

Table of Contents

List of Tables ..........................................................................................................

List of Figures................................................................................................................................ ix

Nomenclature ................................................................................................................................... xii

1 Chapter: Introduction ........................................................................................................ 1

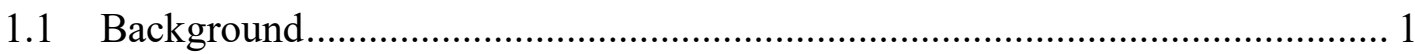

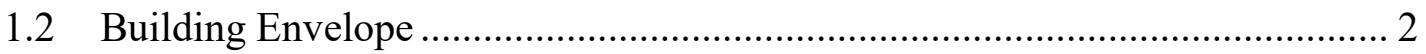

1.3 Common Building Insulation ................................................................... 4

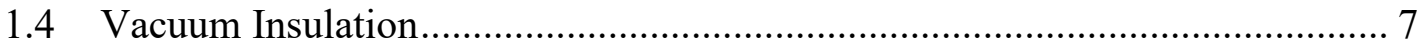

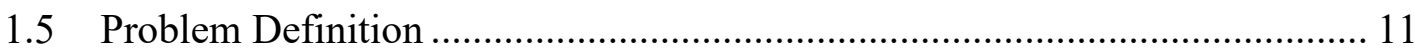

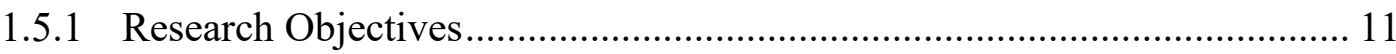

1.5.2 Contributions of Research ...................................................................... 12

1.6 Organization of Research and Thesis Document ......................................... 12

2 Chapter: Literature Review......................................................................................... 14

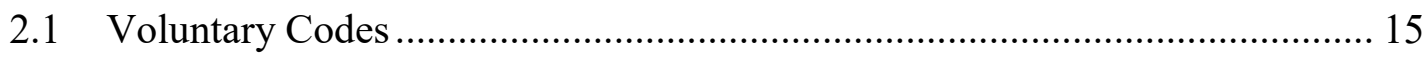

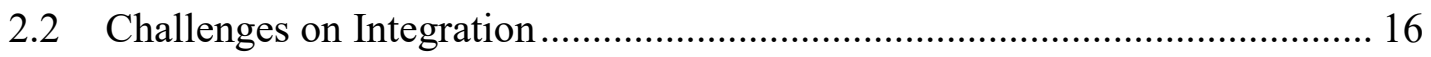




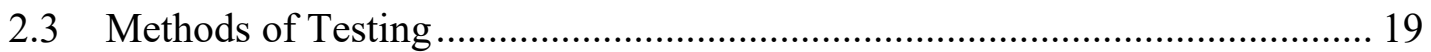

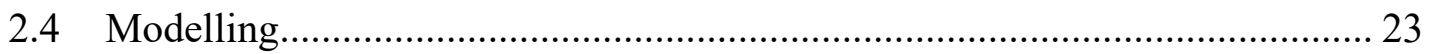

2.5 Vacuum Insulation Properties and Alternatives .............................................. 24

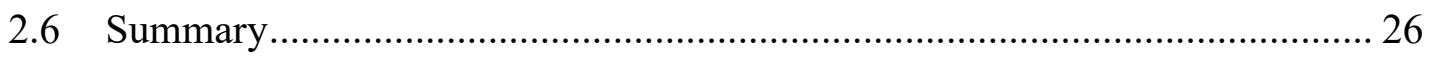

3 Chapter: Experimental Approach............................................................................. 28

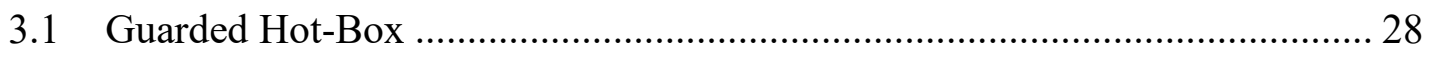

3.1.1 Metering Chamber ................................................................................ 31

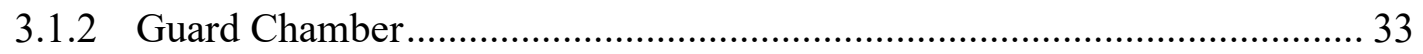

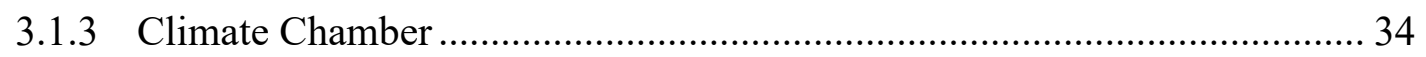

3.1.4 Data Acquisition and Control Systems ……………………...................... 36

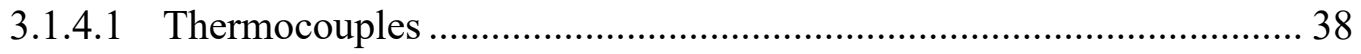

3.1.4.2 Power Monitoring............................................................................... 39

3.1.4.3 Heat Flux Plates.............................................................................. 40

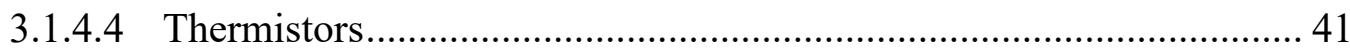

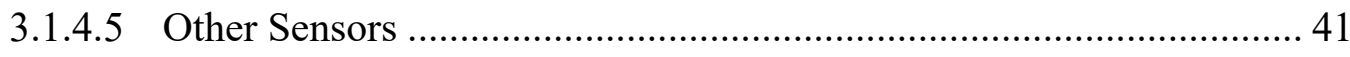

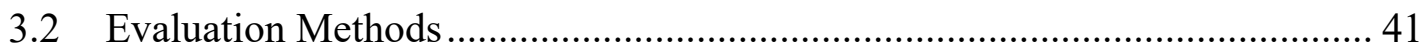

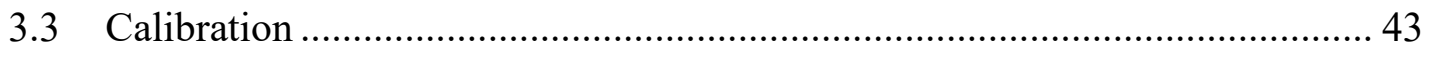

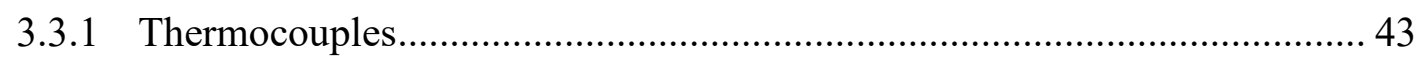

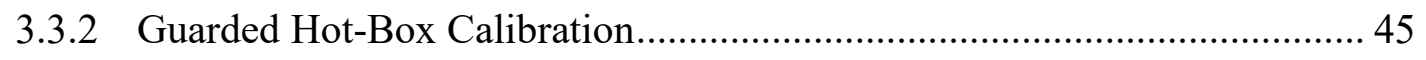




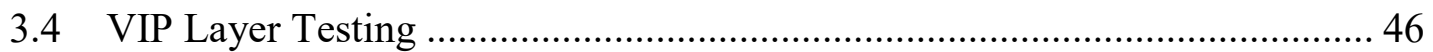

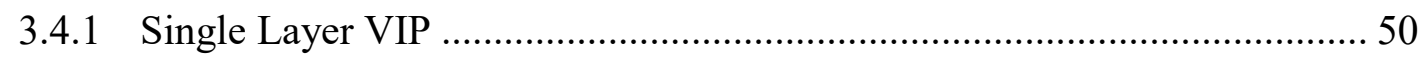

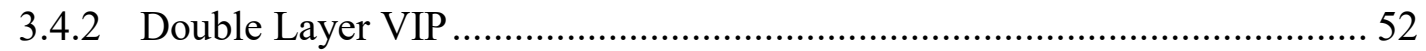

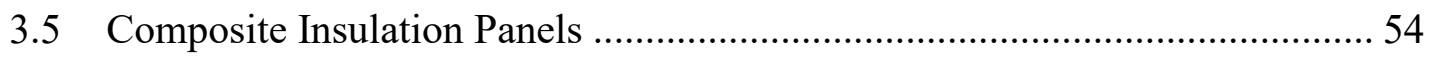

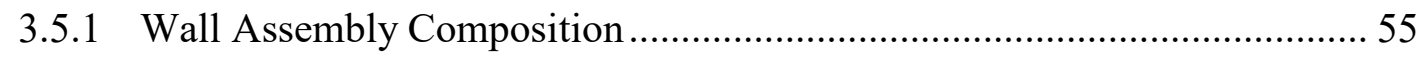

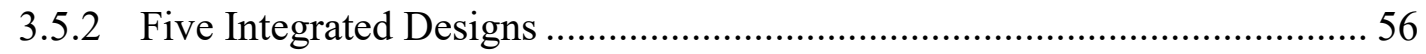

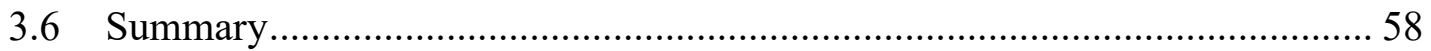

4 Chapter: Modelling Approach....................................................................................... 59

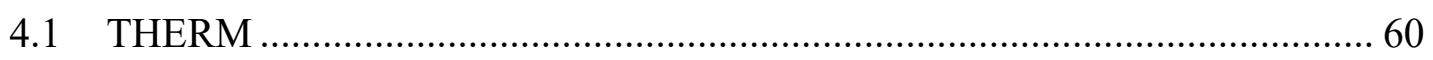

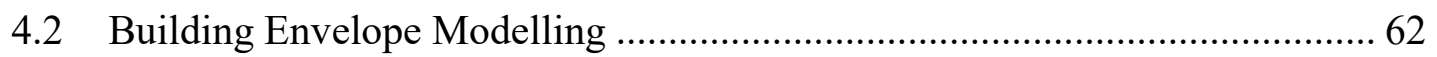

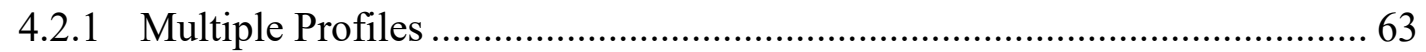

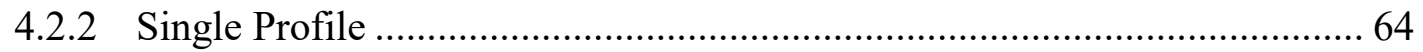

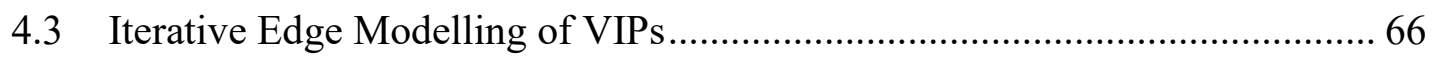

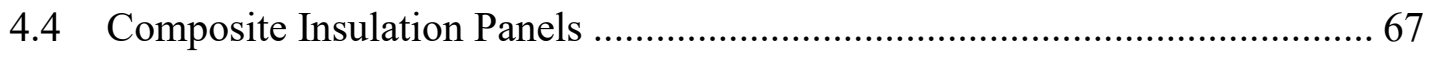

4.5 Summary

5 Chapter: Results............................................................................................................... 70

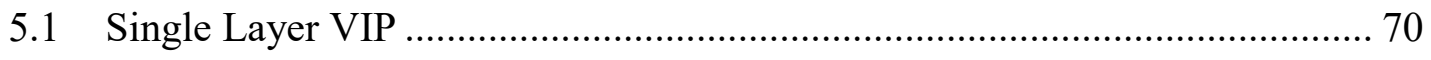

5.1.1 Effective Thermal Resistance of a Single Layer ......................................... 71

5.1.2 Thermal Resistance Center, Corner, and Edge of the Panel ........................ 72 
5.1.3 Creation of a Temperature Heat Map ..................................................... 73

5.2 Double Layer VIP ............................................................................. 76

5.2.1 Thermal Resistance of Each Offset ......................................................... 77

5.2.2 Variation of Heat Flux through Double Layered VIPs............................. 80

5.3 Entire Wall Assembly ................................................................... 83

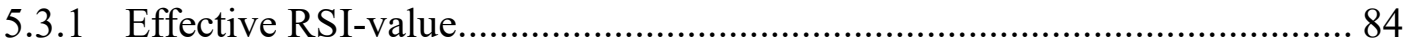

5.3.2 Temperature Profile at Four Cross Sections ….................................... 85

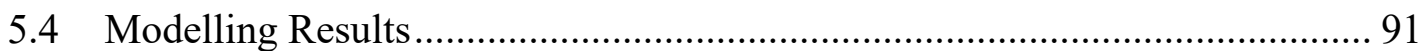

5.4.1 Multiple Profile Simulation .................................................................. 92

5.4.2 Single Profile Method for Non-Homogenous Wall Assemblies ................. 96

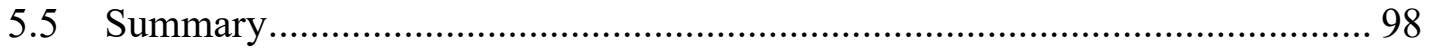

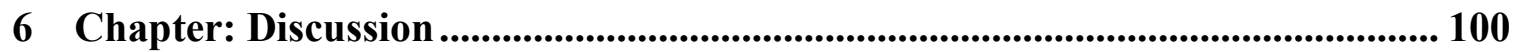

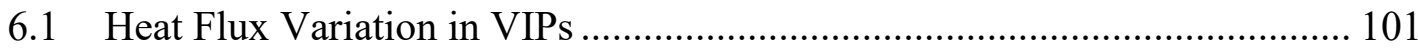

6.2 VIP Offset Effects on Heat Flux and Temperature .................................. 102

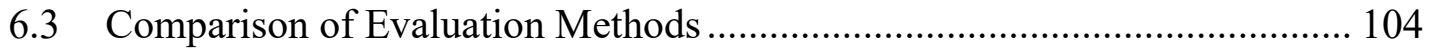

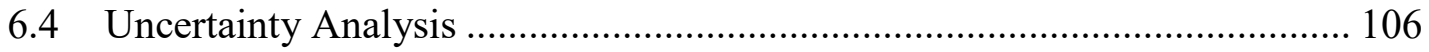

6.5 VIP Integration into the Building Envelope .......................................... 110

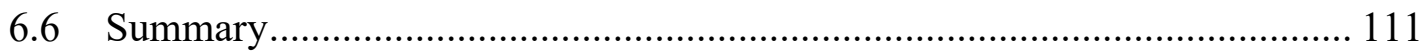

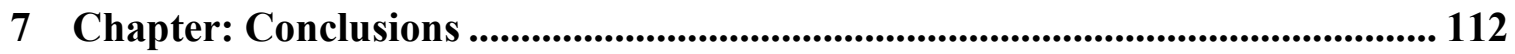


7.1 Configuration Effects in Multiple VIP Layers

7.2 Prefabricated Panel Feasibility ............................................................... 112

7.3 Importance of Proper VIP Dimensions ................................................... 113

7.4 Single Profile Modelling Effectiveness ................................................. 113

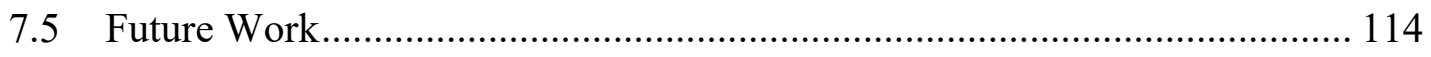

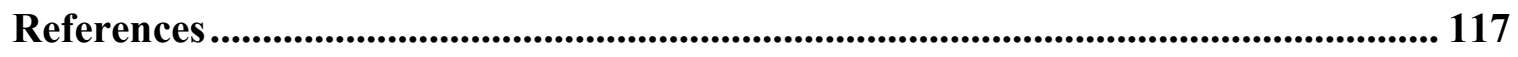

Appendix 1 - Instrumentation Plans for Composite Panels in Guarded Hot-box . 123 


\section{List of Tables}

Table 3-1: Coefficients for calibrated thermocouple equations for each different spool ............. 45

Table 3-2: Calibration data for 5 test periods of the guarded hot-box facility ............................. 46

Table 3-3: Horizontal dimensions to the center of wall for 5 composite wall assembly designs

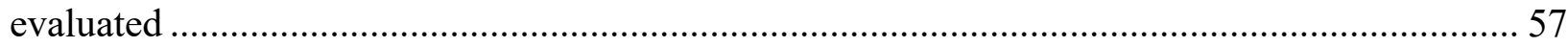

Table 4-1: Material properties used for all the components in the modelling study ..................... 68

Table 5-1: Steady-state test conditions for a single VIP layer....................................................... 72

Table 5-2: Measured temperature and heat flux around VIP .................................................... 73

Table 5-3: Difference in thermal resistance at points on a VIP .................................................. 73

Table 5-4: Test conditions for each double layer VIP configuration........................................... 79

Table 5-5: Summary of steady-state test results over 20 hour period......................................... 85

Table 5-6: Summary of THERM results using weighted average technique ............................... 93

Table 5-7: Coverage area and results for each cross section in 48" single profile simulation ..... 97

Table 6-1: Percent difference between RSI of designs using various evaluation methods ........ 104

Table 6-2: Uncertainties for exterior and interior surface temperature .................................... 108 


\section{List of Figures}

Figure 1-1: A diagram of the general composition of a VIP, adapted from Kalnaes [9].............. 8

Figure 1-2: Comparison of RSI-value per meter of insulation materials................................... 8

Figure 1-3: VIP installed on a building envelope in preparation for the guarded hot-box.......... 10

Figure 3-1: Simplified schematic of Carleton University guarded hot-box ............................ 30

Figure 3-2: Photo of guarded hot-box located at Carleton University..................................... 30

Figure 3-3: Schematic of guarded hot-box in secondary set-up when capturing infrared images 31

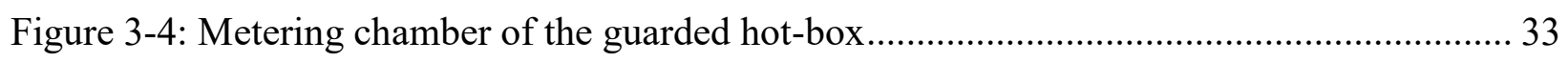

Figure 3-5: Quick connect thermocouples installed inside the climate chamber ..................... 36

Figure 3-6: Photo of data acquisition and control systems used for guarded hot-box................ 37

Figure 3-7: Photo of Delta controller integrated with the guarded hot-box ............................. 37

Figure 3-8: Data acquisition unit used for guarded hot-box with instrumentation connected...... 38

Figure 3-9: A compromised VIP layer featuring two broken VIPs ..................................... 49

Figure 3-10: Single layer VIP affected by fan blowing directly on specimen surface ............... 49

Figure 3-11: Single layer VIP layout within surround panel with dimensions......................... 51

Figure 3-12: Labeled diagram of embedded instrumentation layout on the warm side of a single

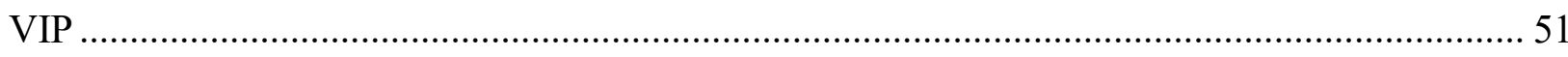

Figure 3-13: VIP layout of second layer offset to center-seam orientation ............................. 53

Figure 3-14: VIP layout of second layer offset to center-corner orientation ........................... 53

Figure 3-15: Construction of a composite panel with VIPs encased in XPS with top sheet removed 55

Figure 3-16: Example composition of wall assembly utilizing composite panels with VIPs. .... 56

Figure 3-17: Legend for dimensions of 5 wall assembly designs provided in Table 3-3 _.......... 57 
Figure 4-1: U-factor result interface in THERM software 62

Figure 4-2: Real geometry of a code-built building envelope in THERM 63

Figure 4-3: Simplified geometry of a code-built building envelope using a fictitious stud size in THERM. 63

Figure 4-4: Layout of single profile simulation that includes two fictitious stud sizes and coverage areas 66

Figure 4-5: THERM cross sections of Test 3 using. Top is only XPS, bottom is VIP with XPS spacers. 68

Figure 5-1: Single layer VIP configuration inside guarded hot-box........................................... 72

Figure 5-2: Thermocouple layout behind a single VIP without thermocouples ........................... 74

Figure 5-3: Thermocouple layout behind a single VIP with thermocouples. 74

Figure 5-4: VIP heat map using Microsoft Excel with measured temperatures are indicated by the white numbers 76

Figure 5-5: Steady-state temperature profile at each material interface of center-center configuration...... 78

Figure 5-6: Steady-state temperature profile at each interface of center-seam configuration ...... 78 Figure 5-7: Steady-state temperature profile at each material interface of center-corner configuration...... 79

Figure 5-8: Difference between maximum and minimum heat flux over the test period.............. 81

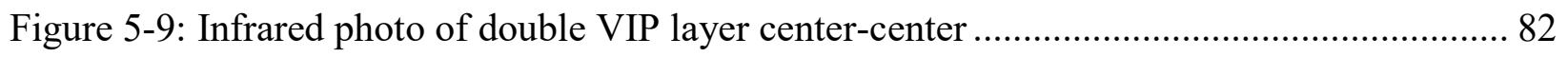

Figure 5-10: Infrared photo of double VIP layer center-seam offset............................................ 82

Figure 5-11: Infrared photo of double VIP layer center-corner offsets ....................................... 83

Figure 5-12: Steady-state temperature profile at the VIP-stud cross section of Test 1 ................ 86 
Figure 5-13: Steady-state temperature profile at the VIP-cavity cross section of Test 1 86

Figure 5-14: Steady-state temperature profile at the XPS-stud cross section of Test $1 \ldots \ldots \ldots \ldots . . . .87$

Figure 5-15: Steady-state temperature profile at the XPS-cavity cross section of Test $1 \ldots \ldots \ldots . . .87$

Figure 5-16: Steady-state temperature profile at the VIP-stud cross section of Test 2 ............. 88

Figure 5-17: Steady-state temperature profile at the VIP-cavity cross section of Test 2 ........... 88

Figure 5-18: Steady-state temperature profile at the XPS-stud cross section of Test 2 ............. 89

Figure 5-19: Steady-state temperature profile at the XPS-cavity cross section of Test 2 ...........89

Figure 5-20: Steady-state temperature profile at the VIP-cavity cross section of Test 5 ........... 90

Figure 5-21: Test 1 cross section incorporating VIPs (top) and isotherm profiles (bottom)....... 94

Figure 5-22: Test 2 cross section incorporating VIPs (top) and isotherm profiles (bottom)....... 94

Figure 5-23: Test 3 cross section incorporating VIPs (top) and isotherm profiles (bottom)....... 94

Figure 5-24: Test 4 cross section incorporating VIPs (top) and isotherm profiles (bottom)....... 95

Figure 5-25: Test 5 cross section incorporating VIPs (top) and isotherm profiles (bottom)....... 95

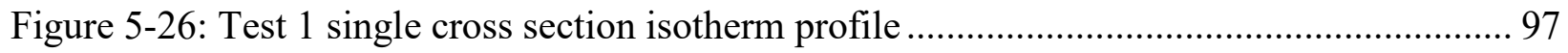

Figure 5-27: Test 2 single cross section and isotherm profile .......................................... 98

Figure 5-28: Test 3 single cross section isotherm profile (bottom) ..................................... 98

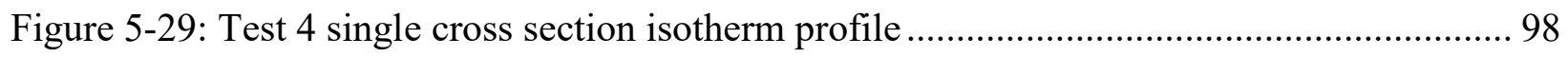

Figure 5-30: Test 5 single cross section isotherm profile ............................................... 98

Figure A-1: Section view of Test 1 wall assembly with composite panels ........................... 123

Figure A-2: Section view of Test 2 wall assembly with composite panels .......................... 124

Figure A-3: Section view of Test 3 wall assembly with composite panels ........................... 125

Figure A-4: Section view of Test 4 wall assembly using composite panels.......................... 126

Figure A-5: Section view of Test 5 wall assembly using composite panels.......................... 127 


\section{Nomenclature}

Symbol

A

$B$

E

$q^{\prime \prime}$

$N$

$P$

RSI or $R$

$T$

$t$

$U$

V

Subscript

$\mathrm{C}$

eff

$\mathrm{H}$

i

average
Description

Area

Bias

Energy input to the metering chamber

Heat flux

Number of measurements

Precision

Thermal resistance

Temperature

Time

Uncertainty

Voltage

Description

Cold surface of a material of wall

Effective thermal resistance

Hot surface of a material or wall

Individual location

Averaged value
Units

$\mathrm{m}^{2}$

watt·hours

$\mathrm{W} / \mathrm{m}^{2}$

$\mathrm{m}^{2} \mathrm{~K} / \mathrm{W}$

${ }^{\circ} \mathrm{C}$

h

$\mathrm{mV}$ 


\section{Acronyms}

\begin{tabular}{|c|c|}
\hline ASTM & American Society for Testing and Materials \\
\hline BATT & Batt insulation \\
\hline CJC & Cold junction compensation \\
\hline EPS & Expanded polystyrene \\
\hline GFP & Gas filled panels \\
\hline GOST & Gosstandart Standards \\
\hline HAM & Heat, air and moisture \\
\hline $\mathrm{HF}$ & Heat flux plate \\
\hline $\mathrm{ICF}$ & Insulated concrete forms \\
\hline IRT & Infrared thermography \\
\hline ISO & International Organization for Standardization \\
\hline NRCan & Natural Resources Canada \\
\hline PLY & Plywood \\
\hline RTD & Resistance temperature detector \\
\hline VIP & Vacuum insulation panel \\
\hline XPS & Extruded polystyrene \\
\hline
\end{tabular}




\section{Chapter: Introduction}

\subsection{Background}

In Canada, energy consumption in the residential sector accounts for $16.5 \%$ of all secondary energy use and $14 \%$ of all greenhouse gas emissions [1]. Of this, space heating and cooling accounts for $63 \%$ of energy consumption and greenhouse gas emissions, which was greater than the all greenhouse gas emissions from passenger cars in Canada in 2011. The energy consumption trends in Canada resembled different regions across the globe, as buildings in the United States and Europe account for $40 \%$ and $38 \%$ of their energy use respectively. When analyzing the energy use in the residential sector, space conditioning accounts for the greatest percentage and varies between $49 \%$ and $64 \%$ across the listed regions. These statistics suggest that reducing the amount energy used for space conditioning can have the greatest impact on the energy performance of a building.

An occupied building consumes energy to provide a conditioned environment for people to live or work. These energy requirements for space conditioning maintain a level of indoor comfort through mechanical heating, ventilation and air conditioning equipment. At the same time, the building is constantly exchanging energy through the building envelope, such as exterior wall, attic, roof, and basement. The space conditioning loads required from the mechanical equipment are closely related to the energy exchange through the building envelope. These loads may be reduced through enhancements to the dwelling's mechanical equipment or building envelope.

The mechanical equipment consumes the energy to maintain the indoor comfort. Reducing the energy use through increased efficiency of mechanical equipment or thermal storage is one route. However, through the efforts of the past decade, commonly used mechanical equipment such as boilers, furnaces, heat pumps and air conditioners have begun approaching their theoretical 
limits of performance. This leaves little ability to address space conditioning energy consumption through increased mechanical efficiency in buildings. As a result, engineers and building designers need to find alternative methods to improved building energy performance while maintaining cost-effectiveness.

\subsection{Building Envelope}

The building envelope offers a barrier between the indoor and outdoor conditions and can suppress the energy changes within the indoor environment. A cost-effective and common practice to improve the energy performance is by improving the building envelope with added insulation. With the added insulation, the building envelope has a higher thermal resistance value and can reduce the amount of energy transfer into and out of the conditioned space. As such, with a more thermally resistive building envelope, the space requires less mechanical heating or cooling energy to compensate for the building's interaction with the exterior environment. Building envelope improvements offer one of the most effective methods for reducing the space conditioning loads, and therefore affect the energy consumption and greenhouse gas emissions in the residential sector. However, insulation may not be continuously added as there are limits to the allowable envelope thickness and the return on investment diminishes as more insulation is added [2].

It is possible to increase the amount of insulation within the building envelope to improve the effective thermal resistance (RSI-value). When increasing the building envelope thickness, the envelope occupies more space within or around the dwelling. Common building insulation materials, such as mineral wool, fiberglass, or foam board, are often used. When insulation is added to the interior side of the sheathing, the interior floor area of the dwelling is sacrificed. The RSI-value enhancements inboard are achieved through larger studs to provide a thicker cavity for insulation. For example, changing the stud construction from $38 \mathrm{~mm}$ by $89 \mathrm{~mm}(2 \times 4$ lumber) to 
$38 \mathrm{~mm}$ by $191 \mathrm{~mm}$ ( $2 \times 8$ lumber), doubles the thickness of cavity insulation available. Moving the interior boundary by $102 \mathrm{~mm}$ (4") can have a significant effect on the price per square foot of floor area value. For example, in 2012, the floor area value to the builder was $\$ 200$ per square foot [3]. Adding insulation to the exterior of the dwelling can conserve the interior floor area valued by builders and homebuyers. This would be beneficial to increase the thickness to $101 \mathrm{~mm}$ (4") of foam board along the outside perimeter of the building as opposed to using $25.4 \mathrm{~mm}$ (1"). However, adding insulation extends the boundaries of the dwelling closer to the property lines. This may not be feasible since new homes are consistently built to the minimum setbacks of the lot. The envelope thickness, interior and exterior space requirements, and diminishing returns as the RSI-value increases are factors that need to be considered when improving the thermal performance of the building envelope.

When designing a building envelope, the local building codes provide a minimum required standard. With recent developments in the building codes locally and globally, a larger emphasis has been placed on building energy efficiency and reduction in their overall energy consumption. Among these codes is the prescribed minimum effective RSI-value for the building envelopes, which are related to the climate zone that the building is situated. A climate zone is a region with similar meteorological conditions, and in the case of prescribed insulating levels, is predominantly based on the heating and or cooling degree days for a given location. The International Energy Conservation Code, developed by the International Code Council, are a global authority that many local building codes use for the basis of their own codes, offer different effective RSI-values for two opposite climate zones. In a warm and temperate climate, such as Southeastern United States, the minimum prescribed effective RSI-value is $2.8 \mathrm{~m}^{2} \cdot \mathrm{K} / \mathrm{W}\left(15.8\left(\mathrm{hr} \cdot{ }^{\circ} \mathrm{F} \cdot \mathrm{ft}^{2}\right) / \mathrm{BTU}\right)$ compared to a $4.9 \mathrm{~m}^{2} \cdot \mathrm{K} / \mathrm{W}\left(27.7\left(\mathrm{hr} \cdot{ }^{\circ} \mathrm{F} \cdot \mathrm{ft}^{2}\right) / \mathrm{BTU}\right)$ for a cold, heating dominated climate. The discrepancy 
between the minimum prescribed effective RSI-values highlights the impact that climate has on the building envelope from the large amount of heating or cooling degree days. It also shows the impact the building envelope can have on space conditioning loads. With a higher number of heating and cooling degree days, the more viable a thermally resistive envelope becomes.

Building envelope upgrades have been performed in practice by Natural Resources Canada's (NRCan) ecoENERGY Innovation Initiative [2]. In partnership with five homebuilders across Canada, the project objective was to show the feasibility of Net Zero Energy Homes by constructing demonstration homes in different locales in Canada. A common trait through these homes was upgrading the building envelope to a thermal performance between RSI-5.2 $\mathrm{m}^{2} \cdot \mathrm{K} / \mathrm{W}\left(\mathrm{R}-30 \mathrm{hr} \cdot{ }^{\circ} \mathrm{F} \cdot \mathrm{ft}^{2} / \mathrm{BTU}\right)$ and RSI-7.04 $\mathrm{m}^{2} \cdot \mathrm{K} / \mathrm{W}\left(\mathrm{R}-40 \mathrm{hr} \cdot{ }^{\circ} \mathrm{F} \cdot \mathrm{ft}^{2} / \mathrm{BTU}\right)$ effective, well above code-built homes today [4]. Upgrading the building envelope to achieve a certain performance designation includes a lower heat loss or gain of the dwelling, thereby lowering the space heating and cooling demand. This may also lead to a smaller capacity requirement for the mechanical systems inside the dwelling, potentially reducing the initial cost of investment of the home.

\subsection{Common Building Insulation}

Many factors contribute to the selection of which insulation material to use in the buildings. Buildings are insulated with a variety of materials that use different types of fastening methods, air and vapour permeability, and their different thermal insulating properties, specifically RSI-values (R-values) or thermal conductivities. Conventional building insulations can be found in different forms. They are available as a loose wool, a blanket roll, rigid foam board, spray foam, or integrated into structural materials. The benefits and advantages can include the way they are 
installed, their RSI-value per unit thickness, where in the envelope they are located, and their thermal, moisture, and air leakage properties.

The most common insulation found in buildings, according to the US Department of Energy [5], is blanket or batt insulation. It is composed of flexible fibers such as fiberglass, mineral wool, plastic or natural fibers. They are available in widths and thicknesses that are suitable for standard spacing of studs and joists outlined in building codes. The insulation is typically installed within unfinished walls, and floor and ceiling joists where they can be fitted inside very easily to the standard spacing. However, if the spacing and insulation width do not align, the batt or roll can be hand-cut or altered on-site easily without consequence. The RSI-value of these batts are related to their density, and may change the RSI-value from RSI-1.94 $\mathrm{m}^{2} \mathrm{~K} / \mathrm{W}$ lower density fiberglass batt to an RSI-2.64 $\mathrm{m}^{2} \mathrm{~K} / \mathrm{W}$ for a high-density fiberglass batt for a $89 \mathrm{~mm}(3.5$ ") thick material. The effective RSI-value of the building envelope may be improved by increasing the thickness. However, the building envelope will grow thicker as a whole, since the RSI-value for these materials are as high as $32 \mathrm{~m}^{2} \cdot \mathrm{K} / \mathrm{W}$ per meter.

Foam board insulations are also common in buildings applications. They are a rigid panel of insulation and can be easily fastened to many facets of the building to increase the thermal resistance. The product is typically composed of either polystyrene or polyurethane and is capable of providing a RSI-value of 27.6 to $45.0 \mathrm{~m}^{2} \cdot \mathrm{K} / \mathrm{W}$ per meter ( 4 to $6.5 \mathrm{hr} \cdot{ }^{\circ} \mathrm{F} \cdot \mathrm{ft}^{2} / \mathrm{BTU}$ per inch) [6]. The insulation can be added to the building, in areas such as the subfloor, exterior wall, interior wall, foundation wall, attic and roof. The foam board's manufacturing process greatly affects its moisture transfer properties. For example, two common foam boards manufactured from polystyrene are extruded polystyrene (XPS) and expanded polystyrene (EPS). The XPS is manufactured using a continuous extrusion process that creates a vapour tight foam, also referred 
to as a closed-cell structure. The EPS is manufactured by exposing small foam beads to high heat and pressure in order to expand the beads. This will leave small paths between the beads for moisture to penetrate or build up, unlike the XPS and can be referred to as an open-cell structure. However, the materials yield a similar RSI-values per unit thickness. As such, using these materials to upgrade a building envelope to meet the high performance standards available would not provide a thin building envelope assembly.

Other options for building insulation exist in the market, but are not common to residential construction in Canada due to their cost or complexity. The usage of insulated structural materials, such as insulated concrete forms or structural insulation panels, have been on the rise in an effort to improve the thermal efficiency of homes. Insulated concrete forms (ICFs) offer a significant thermal improvement for basement and foundation walls compared to standard construction. ICFs have two added layers of foam insulation around the concrete to provide the benefits of plain concrete and improved thermal performance while maintaining the appearance of a standard building [7]. Insulation Structural insulation panels may be seen in modular or prefabricated home since can require a mere three days for construction. The panels are structural panels with insulation contained inside offering the structural and insulation requirements for the building. The panels are connected at the edges and are capable of making an air and moisture tight seal. The panels are typically packaged as a prefabricated kit that can be completely constructed within a few days. By providing the structural load in addition to the prescribed insulation in a single material, the dwelling can maintain or improve the thermal efficiency of the envelope.

These insulations may be used together to make the building envelope a comprehensive insulator for the building. The foam board insulation and batt insulation can be installed on separate sides of the sheathing. The foam board is added to the exterior of the structural sheathing to provide 
a continuous layer of insulating material. Meanwhile, the batt insulation is placed between each of the structural studs at the interior of the sheathing. The composition of $25.4 \mathrm{~mm}$ (1") of continuous exterior insulation, and $89 \mathrm{~mm}(3.5$ ") of insulation in the cavity can usually achieve a minimum prescribed effective RSI-value for buildings in the majority of Canada. However, when designing a thin, high RSI-value building envelope of RSI $6.82 \mathrm{~m}^{2} \mathrm{~K} / \mathrm{W}$ with minimal increase to the thickness, using conventional building materials may not be the solution and higher performance materials need to be examined.

\subsection{Vacuum Insulation}

As the market and government incentives drive a more sustainable future, it is apparent that conventional insulation methods are insufficient toward attaining a thin, high RSI-value building enclosure. Vacuum insulation panel (VIP) is a porous material core that has been contained within a metallic foil with nearly all the air evacuated and sealed, shown schematically in Figure 1-1. Evacuating the VIP to a pressure of approximately 10 mbar effectively eliminates convection and conduction through the center of the panel [8]. The low pressure and structure enables the panel to yield a high thermal resistance per unit thickness, which could help reduce the energy used for space conditioning of buildings. VIPs offer a high RSI-value value per unit thickness value that can exceed fiberglass batt insulation by 10 times [8]. As for other commonly used insulations, Figure 1-2 compares the typical RSI-value per meter for each material. In Figure 1-2, the thermal resistance per meter thickness for various insulations used in buildings and highlights the advantages of VIPs compared to batt insulation installed between studs. 


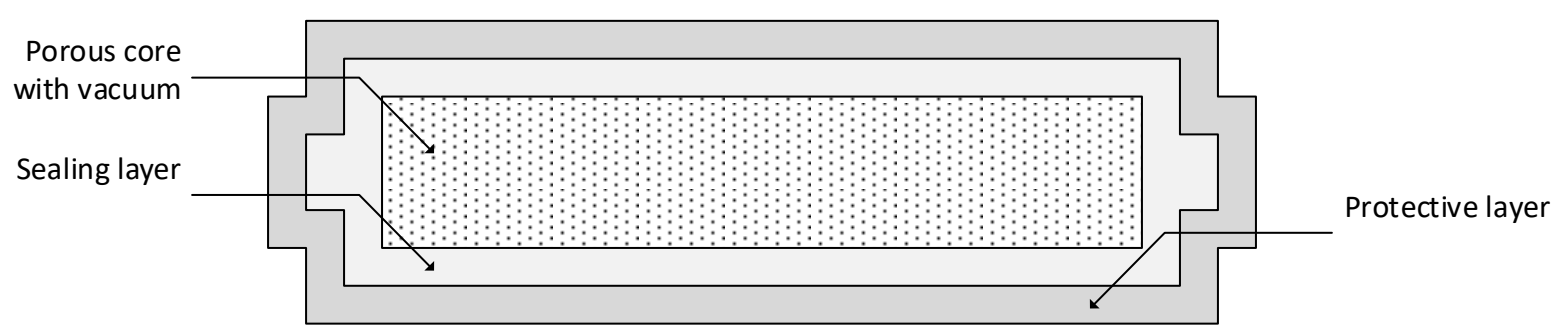

Figure 1-1: A diagram of the general composition of a VIP, adapted from Kalnaes [9]

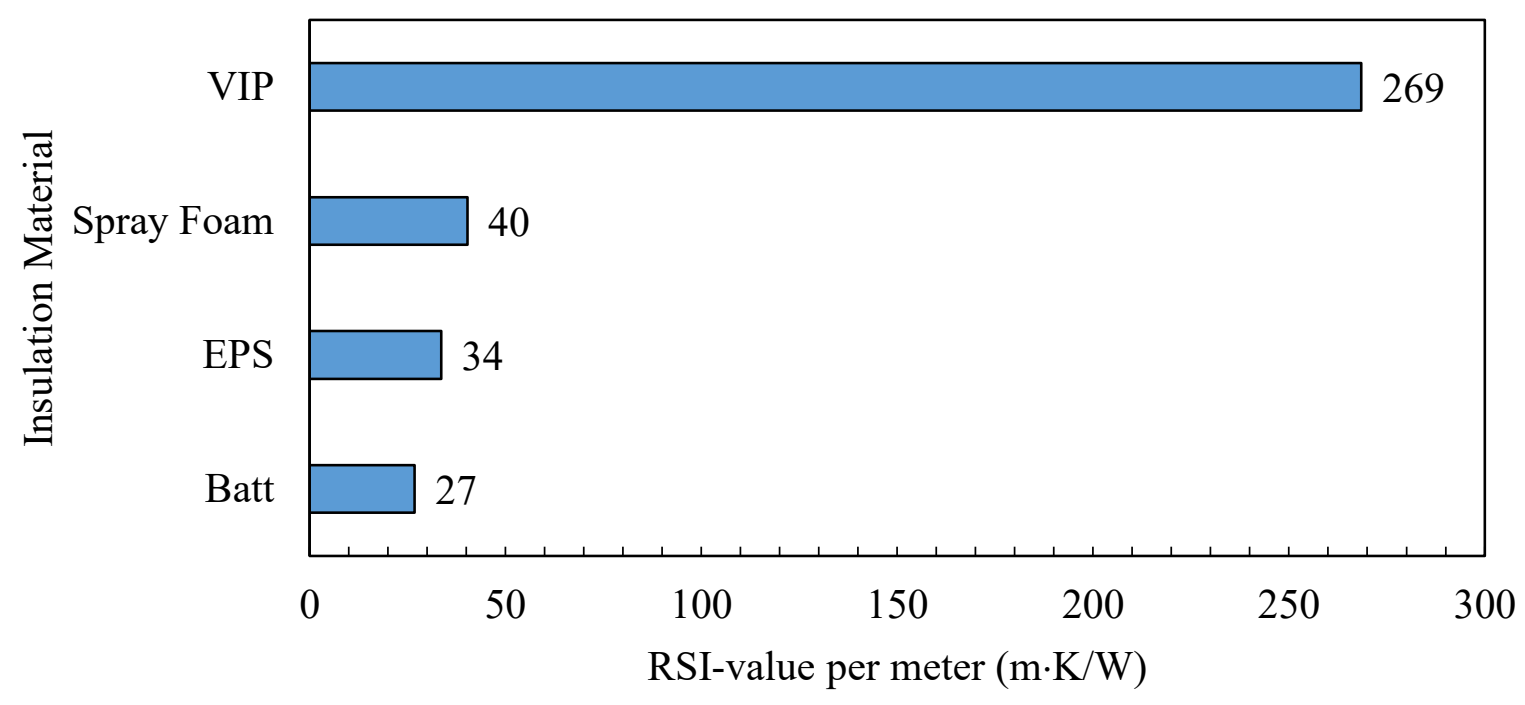

Figure 1-2: Comparison of RSI-value per meter of insulation materials

VIPs can be implemented into the building envelopes to maintain current minimum code-built RSI-values for residential homes with in a thinner building envelope thickness, or can be used to achieve a high performance building envelope without increasing the envelope thickness [10]. By integrating this high performance insulation into the building envelope, high RSI-values can be achieved with thin wall envelope profiles, saving on space and energy. VIPs could be integrated into building envelope designs since new residential buildings are being constructed with the minimum required clearance from property lines to maximize the interior floor area. The useful floor area savings should be considered in the economic analysis of VIPs implemented in residential buildings. One study found the product and installation costs for 
$30 \mathrm{~mm}$ VIPs approached $84.5 \mathrm{USD} / \mathrm{m}^{2}$ of exterior wall [10], which is significantly higher than the cost to install conventional insulations. This indicates that an economic analysis should evaluate both energy and space savings.

While vacuum insulation technology has the potential to impact the residential sector, significant obstacles exist before widespread implementation can occur. Since an important property of VIPs is the internal pressure within the foil, a puncture that results in a sudden or slow increase in pressure will result in performance reduction. VIPs also have non-homogenous RSI-values across its face which characterizing the overall thermal performance of the VIP during modelling approaches.

The thermal performance benefits are nearly all lost if the panel is compromised or punctured such that a loss of vacuum occurs. As such, VIPs are fragile in nature and must be handled and integrated with care, which is uncommon for building insulating materials. Quality control measures need to be in place during panel manufacturing, transportation, implementation of VIPs, and installation of other building components. This issue is accented by the details needed to finish the building with siding or other penetrations. The building details and VIP layout need to be carefully planned to not waste any VIPs, but also maximize the wall coverage of VIPs. An example of a VIP layer is shown in Figure 1-3, where 12 VIPs are utilized. Note the gap between the two rows represents the fastening area necessary to the finish the exterior of the envelope. Currently, VIPs are predominantly implemented into refrigeration appliances, such as freezers, refrigerators, and shipping containers. Unlike residential construction, in these markets the VIPs are installed in a controlled factory setting that significantly reduces the risk of damage during manufacturing. Therefore, before VIPs can become commonplace in residential home market, the risk of damage from the hazards of a construction site needs to be addressed. 


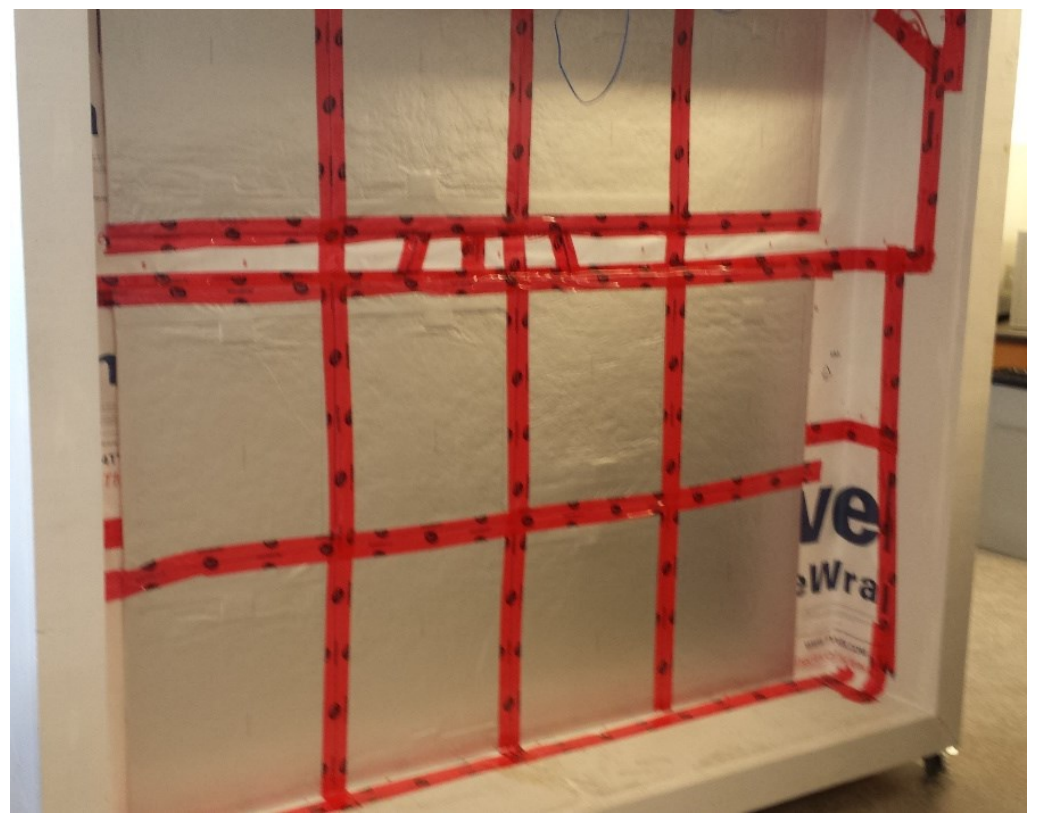

Figure 1-3: VIP installed on a building envelope in preparation for the guarded hot-box

In addition, thermal bridging or non-homogenous effects exist along the perimeter of VIPs. While the exact amount can vary between type and size of VIP, a deviation in RSI-value exists between the center and seams of the VIP. This thermal bridge significantly hinders the effective thermal performance of the panel and the wall assembly as a whole. When implemented into a building assembly, the perimeter of the VIPs causes paths for heat to travel and create cold spots on the sheathing. The non-homogenous property also increases the difficulty modelling the thermal and hygrothermal performance of a building envelope with VIPs. Utilizing the center of panel RSI-value value during a simulation yields an overestimate. Assigning an effective RSI-value to the VIP that encompasses the center and edge values does not provide a proper isothermal profile.

These isothermal profiles are important when attempting to evaluate how the temperature propagates through the assemblies, which can vary depending on whether a seam is aligned with the stud or cavity of the wall assembly. The temperatures at these surfaces are related to the dew point within the wall affecting the long-term feasibility of the design. The issue with evaluating 
the moisture effects from these systems is the length of time required to validate concerns or simulation results with experimental data. The experimental data taken from in-situ test conditions must be taken from multiple seasonal cycles to assess the trends and its drying potential over time. These exercise caused the research scope of this completed study to be limited to the thermal effects of VIPs into residential buildings envelopes.

\subsection{Problem Definition}

Residential energy consumption, specifically space heating and cooling, is an area that can greatly affect the efforts to reduce energy requirements. The building envelopes, at the current standard with conventional insulating materials, cannot achieve the RSI-value necessary without significantly increasing the envelope thickness. The overall goal of this research was to develop a thin, high RSI-value building envelope that is suitable for residential housing in Canada. This goal was met with the use of VIPs because of their thermally resistive properties. However, before VIPs can be integrated into buildings for widespread use to reduce energy consumption of a home, better modelling and creative implementation are required. Since fragile and thermally non-homogenous materials are not conventionally used within building envelopes, it is important to develop an effective method to evaluate the effective RSI-value through modelling and design against potential failure. The topic of this thesis addresses the design and evaluation of single and multi-layered VIP envelope assemblies for residential buildings.

\subsubsection{Research Objectives}

The objectives of this study were to:

1. evaluate and quantify the reduction in thermal resistance that VIPs will experience at the edge of the panel; 
2. determine the effects of offsetting a second layer of VIPs on the overall thermal resistance and the variations in heat flux and surface temperature;

3. develop a heat map using Microsoft Excel spreadsheets to visualize the surface temperatures at the VIP interface;

4. evaluate the feasibility and effectiveness of encasing VIPs within other insulations as a protection technique;

5. develop a new modelling approach to study wall assemblies containing multiple unique cross sections and;

6. compare the experimental results to the model to verify the new approach;

\subsubsection{Contributions of Research}

The outcomes of this work have:

1. revealed the distance from the edge where the center of panel properties are valid;

2. resulted in a quantified value of loss in thermal resistance at the edge of a VIP;

3. resulted in offsetting VIPs to reduce the surface temperature variations;

4. produced a heat map in Microsoft Excel to visualize VIP interface temperature data from steady-state conditions;

5. assessed the feasibility of VIPs encased in insulation as a technique to protect VIPs and improve the thermal performance of a building envelope;

6. developed and validated new and existing modelling techniques for wall assemblies incorporating VIPs or non-homogenous properties.

\subsection{Organization of Research and Thesis Document}

The research presented in this document has been performed over a period of two years. Over this time, some of the content and results have been presented at peer-reviewed conferences. 
The thesis represent a compilation of results presented in these papers and have been referenced throughout.

The approach taken during the research focused on the experimental evaluation of the RSI-value at the edge of VIPs and their integration into a full wall assembly. To determine the effectiveness of the new modelling method, full scale building envelope assemblies were built in laboratory and tested in a guarded hot-box to verify the simulated results.

Chapter 1 provides a background on vacuum insulation panels and their application into buildings. It also outlines the scope and objectives of the thesis.

Chapter 2 presents previous research considered relevant to this study.

Chapter 3 describes the experimental apparatus used to evaluate the wall assemblies using VIPs at steady-state conditions and describes the sequence of designs that were tested.

Chapter 4 presents the modelling methodologies developed to evaluate the edge effects of VIPs and the integration into a wall assembly.

Chapter 5 presents and summarizes the results of the experimental evaluation of VIPs individually and as a system.

Chapter 6 provides a discussion and analysis of the experimental results, feasibility of certain systems and the effectiveness of the new modelling methodologies.

Chapter 7 presents conclusions and recommendations based on the work described in the previous chapters, and makes recommendations for future areas of study. 


\section{Chapter: Literature Review}

This chapter contains a literature review of past and current research of projects based on the use of VIPs in new and retrofitted building assemblies and the evaluation of VIP thermal performance and service life. Different means of fabricating and implementing VIPs within building assemblies are discussed. Current methods of evaluating VIPs on a singular panel basis and the effective building assembly are presented in detail. Finally, a review of current modelling practices for non-homogeneous assemblies incorporating VIPs is discussed.

Previous studies on VIPs have focused on the different fabrication methods, configurations, size of panels and their relationship with thermal and moisture performance. The applications that are frequently mentioned are the building envelope, refrigeration appliances and transportation. Multiple quantitative and qualitative methods have been described and used to evaluate the thermal and moisture performance by steady-state, in-situ, or test bench apparatuses. Quantitative methods for determining the effective RSI-value are using in-situ set-ups a guarded hot plate set-up or a steady-state guarded hot-box facility. The qualitative methods such as infrared thermography (IRT) are used in conjunction with the other methods to find the thermal bridges, surface temperature distributions, or quality assurances after construction. There are modelling and simulation requirements for building performance programs that have been developed such as R-2000, Passive House and LEED standards [12, 13, 14], especially when uncommon materials are introduced to the design. These requirements are difficult due to the non-homogenous nature of VIPs. When the VIPs are treated as a homogenous layer within the envelope with center of panel RSI-value, the effective RSI-value of the building envelope will be greater than reality. However, when the RSI-value of the edge is used, the effective RSI-value of the envelope will be lower than reality. 


\subsection{Voluntary Codes}

Since energy conservation has become an issue in society, building standards and performance have been shifted towards energy efficiency. These standards have varying requirements that could include builder training, energy consumption limitation and performance levels with respect to heating, ventilation and air conditioning (HVAC) systems and building effective thermal resistance among others.

R-2000 [12] is a technical standard for energy efficiency, air tightness, and environmental responsibility in residential construction. It has become the benchmark for energy efficient new buildings across Canada. The series of performance requirements, in addition to building codes, are highlighted by the builder's license and the specific energy budget. Builders need to have completed the R-2000 training required in order to build homes that can be certified. Since the standard is continually changing to incorporate new technologies, training is provided as said changes occur as incentive to remain involved in the program. The R-2000 certified homes must operate within an energy budget, usually required to operate with less than $30 \%$ of energy required to operate code-built homes of the same size at the same location in Canada. In order to stay within the prescribed energy budget, a combination of energy efficient HVAC systems and an increase in building envelope thermal resistance are integrated to the new or retrofitted homes. VIPs can be implemented into the building enclosure to improve the effective thermal resistance and obtain the high performance designation.

The Passive House designation through Canadian Passive House Institute (CanPHI) [13], and although it was originally developed by Passivhaus Institute, it is offered in Canada. The certification criteria is a quality assurance process that ensures the building is designed to achieve high levels of energy performance and occupant comfort. Therefore, CanPHI strongly 
recommends that builders obtain pre-certification or design stage review to ensure the building meets the requirements. The fundamental building envelope aspects outlined by Passive House are the air tightness, the thermal performance of windows, the insulation level, and a thermal bridge free construction. These aspects are directly related to the heat loss to the outdoors through the building envelope and minimizing that loss will allow the building to meet the significant reduction in energy performance compared to standard construction. VIPs offer an alternative to the method to which walls are insulated, as long as the thermal bridging along the edges are taken into account.

LEED Canada Rating Systems through the Canada Green Building Council [14] is a tiered high performance designation program offered in Canada. Similar to the previous two certifications, the minimum requirements are based on building geometry, orientation and performance parameters among other conditions. However, LEED Canada offers four different degrees of certification unlike the certification programs previously discussed. They have sets of requirements for new constructions, commercial buildings, existing buildings, as well as neighborhoods.

\subsection{Challenges on Integration}

A known issue with incorporating VIPs into residential housing is attempting to reduce the mechanical defects and puncture during construction of wall assemblies on site. The defects and puncture could cause reductions in thermal performance in the short term and long-term since maintaining the vacuum within the VIPs is paramount to the performance.

Brunner et al. [15] studied the deterioration of VIPs encapsulated in EPS applied to a façade in an urban area. The results showed that reductions in performance might be attributed to a lack of quality assurance with respect to the metallic envelope as opposed to a VIP's vulnerability inside the building envelope. The VIPs were removed from the façade after the testing period for 
inspection. Through on-site and laboratory tests, the researchers found that the aluminum foil envelope did not meet the required quality and allowed air leakage through the defects. They stated that VIP manufactures could improve their quality assurance measures during the manufacturing process by monitoring the internal pressure, moisture content inside, and thermal conductivity a period after production to verify there are no defects. They stated that the use of VIPs in building envelopes will increase in the future, as the trend has been increasing in the $2000 \mathrm{~s}$, however, they found that quality assurance issues from production to installation is a limiting factor.

Brunner et al. [16] also studied the challenges of widespread implementation of VIPs into residential buildings for new constructions and retrofits. They noted that the market share as of 2014 of VIPs is approximately $90 \%$ towards refrigeration and transport industries and the remaining located in buildings. They indicate the majority of scientific publications are focused on VIPs that are implemented in buildings and the use of VIPs is increasing in European countries, notably Germany and France. They outline that the majority of research in VIPs has been conducted towards augmenting the core material, barrier foil or integration systems of VIPs and a small amount of research addressed the hygrothermal performance of VIPs.

A review of VIPs was performed by Baetens et al. [17] comprised of an introduction to VIPs, the thermal bridges of individual VIPs and the building envelope, the VIP service life, and VIP's building applications. They present the available analytical and numerical models that currently exist to evaluate the temperature and moisture distributions caused by the thermal bridges along the VIP perimeter. A number of different barrier foils, core materials, and spacer techniques were presented along with typical thermal properties and internal pressure. They also listed the building applications for VIPs that include exterior or interior wall insulation for retrofits or new 
builds, flat roof constructions, pipe or domestic hot water tanks insulations, and sandwiching VIPs into doors and windows.

Berge et al. [18] conducted an experimental analysis into reducing the amount of heat losses in a twin pipe district heating system in Sweden. Their focus was directed toward district heating systems and that approximately $10 \%$ of the supplied energy to the network is attributed to heat losses. They were able to save $12 \%-18 \%$ of the total losses and $29 \%-39 \%$ of supply pipe losses by encasing the supply pipe of the twin pipe system in VIPs and polyurethane foam to keep the panels in place. They used a guarded hot pipe test bench set-up as well as a thermal conductance model to evaluate the heat losses. They obtained an agreement between the two evaluation methods and quantified the potential benefits of high performance insulation in district heating applications.

A study from the National Research Council of Canada [19] investigated adoption of new envelope systems and existing barriers such as buildability and energy performance assessment. They outlined that VIPs were already being used for mainstream industrial cladding systems where the cost and performance must be competitive with conventional systems, commercial building cladding to increase the net gross area of the building and architectural applications for appearance purposes. They concluded that further analysis of the edge effects and surround material to contain vacuum within the VIP was required.

A study in Ottawa by Parek et al. [20] from Natural Resources Canada studied the design, construction, and performance of VIP in a Net Zero Energy home referred to as the Harmony House Project. Their goal was to limit the wall thickness to conventional homes and obtain an RSI-value above the conventional standard. Their preliminary results from modelling the assembly in THERM [21] and WUFI [22], and monitoring the in-situ hygrothermal performance showed that the VIPs would act as a vapour and moisture barrier. THERM is a two-dimensional heat 
transfer finite element modelling software, while WUFI is a finite element modelling software used to analyze the moisture transfer through building assembly. In addition, they found that if the thermal resistance of the VIP is reduced, the dew point within the wall can change and cause moisture accumulation on the outside of the sheathing.

The purpose of certain studies has been the energy savings for a residential building incorporating high RSI-value wall assemblies. Rossi et al. [23] investigated middle latitude locations, such as Italy, they used building case studies, which includes retro-fits and new builds incorporating VIPs, and thermodynamic modelling using EnergyPlus [24] to analyze the energy savings. They found that, when compared to a traditional wall, buildings in middle latitudes could experience an energy saving of $40 \%$ during the winter, $9 \%$ during the summer, and approximately $22 \%$ in total annual energy demand. They also noted that other design factors such as building orientation and window-to-wall ratio influence the energy demand more than the exterior wall composition.

To avoid the problems of puncture during construction, the effectiveness of prefabricated panels has been studied. Voellinger et al. [25] precast two layers of center-center VIPs within concrete in an effort to reduce the concrete thickness from $50 \mathrm{~cm}$ to $35 \mathrm{~cm}$ and evaluate its feasibility in construction. The VIPs were also sheathed by two $4 \mathrm{~cm}$ layers of EPS to protect the VIPs during concrete curing. Furthermore, they implemented the design into a detached house in 2010 and optimized the construction of a larger building, determining that using three different sized modules would align with commonly used construction methods.

\subsection{Methods of Testing}

The thermal properties are dependent on the existing vacuum within the panel. A non-destructive method to determine whether the vacuum still exists is by physically examining 
the panel; ensuring that is still rigid and no punctures exist. However, it is possible that a panel remains rigid while also losing its vacuum. Vom Boegel et al. [26] conducted a study using an integrated microelectronic circuit and antenna embedded within the VIP and a contactless sensor to measure the internal pressure was conducted. The sensor transponder, powered through inductive transmission, was capable of detecting the absolute pressure inside the panel to a few mbar at an accuracy of less than 1 mbar. By measuring this pressure, they were able to perform the quality assurance before and after installing panels into the building envelope. The research group also used the sensor transponders to measure the temperature at specific points, similar to embedded thermocouples in the envelope.

Furthermore, the thermal performance of the building envelope and associated materials can be determined through methods such as in-situ testing using the outdoor conditions, steady-state conditions using a guarded hot-box or infrared thermography (IRT). In-situ testing involves controlling the indoor conditions and exposing the outdoor section of the wall assembly to the natural conditions. Steady-state testing involves maintaining controlled conditions on both sides of the wall assembly. After the installation of new or retrofitted buildings with VIPs, a typical method to view the thermal bridges and effectiveness of the new design is to use infrared thermography. One or more of the test methods have been employed to determine the desired properties in recent studies.

A study performed by Asdrubali et al. [27] looked at the difference between thermal transmittance measurements from different standards using a calibrated hot-box. While testing the thermal transmittance of a steel framed window in a steady-state hot-box, they use three test methods according to three standards: ASTM C1363-05 [28], EN ISO 8990 [29] and GOST 26602.1-99 [30]. Their results concluded that the ASTM and EN ISO standards treated the 
specimen as a "black box", such that the non-homogenous properties were not taken into consideration and an effective value was found. The GOST 26602.1-99 standard allowed the evaluation of the thermal properties of each component individually, providing more information on the strengths and weaknesses of the design however; it required an increased amount of time and complexity.

A study conducted by Mukhopadhyaya et al. [10] investigated the use of the retrofitted VIP wall assembly in a Canadian subarctic climate. The study was intended to depict and provide insight to various long-term performance and constructability issues that exist with the implementation of VIPs. They obtained in-situ measurements on the exterior of an institutional building in Yukon, which was retrofitted with VIPs. While temperature and heat flux sensors were embedded within the wall, IRT was used to detect the weakest points of thermal resistance and to confirm that the VIPs remained intact after construction. The in-situ period was over 3 years and they concluded that there were no apparent changes in thermal performance, but noted that further in-situ monitoring would generate valuable data. During the retrofit, the VIPs were $560 \mathrm{~mm}$ by $460 \mathrm{~mm}$ ( 22 " by 18 ") with a $12 \mathrm{~mm}$ thickness made by Panasonic that was sandwiched between two $52 \mathrm{~mm}$ (1") sheets of EPS installed to a $38 \mathrm{~mm}$ by $140 \mathrm{~mm}$ (2x6 lumber) stud construction using batt insulation.

In some circumstances, infrared images were used to compare buildings, either the same building before and after retrofitting or two different high performance buildings designs. Fricke et al. $[31,32]$ used the thermal images to compare a stock dwelling and a VIP insulated home. The outside surface temperature varied by an unknown amount due to lack of colour scale, however the temperature trend was apparent. The stock home was shown as bright red (hot) temperature depicting the heat loss from the interior of the home. The VIP insulated home contained the 
opposite trend, showing a bright blue (cold) temperature meaning limited heat flow through the envelope and therefore fewer losses. The study reported a temperature difference of $2.5^{\circ} \mathrm{C}$ between the two surfaces when the outdoor temperature was $0^{\circ} \mathrm{C}$ and indoor temperature was $20^{\circ} \mathrm{C}$. The study also showed that the relationship and impact of higher insulation levels in the building envelope could be seen using infrared imaging.

A separate study from Schwab et al. [33] used infrared thermography to compare the performance of two high performance buildings. They compare an ultra-low energy timber building incorporating VIPs to a retrofitted VIP-insulated building envelope. In both images, thermal bridges were seen where mounting of the materials exist, however large spots of higher temperature existed in the retrofitted building exist due to defective VIPs. Meanwhile, the timber building offered the advantage of removing the building outer layer to replace faulty VIPs, unlike the retrofit.

Research efforts have been placed to develop a methodology to produce a quantitative thermal bridge measurement from an infrared thermal image. Bianchi et al. [34] continued to develop a strategy where the indoor air temperature, surface temperature and heat flux through the wall, ground and roof are continuously monitored and compared to the infrared images. The monitored building had a $10 \mathrm{~m}^{2}$ floor area with a South facing window. They calculated a hypothetical heat loss from the wall neglecting any thermal bridges that may exist. They determined that there were nine identified thermal bridges and used the IRT analysis to assign a thermal bridge influence value to the area based on the temperature difference between the homogeneous and non-homogenous wall sections. Using a monitored heat pump system to obtain actual energy consumption, they compared it to the energy values with and without thermal bridges. They found that this method yielded an energy consumption value that was $0.2 \%$ less than 
the measured consumption from the heat pump. They noted that this proposed methodology is useful for in-situ conditions when using IRT and a reliable thermal bridge correction factor can be found.

Zalewski et al. [35] conducted a study with a quasi-steady-state system used in parallel with IRT. A wall with embedded temperature and heat flux sensors was inserted between two climate regulated chambers for 24 hours with $42^{\circ} \mathrm{C}$ and $20^{\circ} \mathrm{C}$ air temperatures in the inside and outside chamber respectively after reaching steady-state. An infrared camera was permanently installed $2.5 \mathrm{~m}$ away from the wall in the outside chamber. The camera took photos during the test to show the thermal bridges within the assembly. A model was developed in TRISCO [36], three-dimensional conductive heat transfer software, to determine the temperature distribution at each interface and was compared to the measured data and IRT. The study showed that local heat flux variations were found using heat flux meters, and that IRT is useful for visualizing local zones of heat flux. The study noted that without heat flux measurements, IRT could not provide any quantitative assessments since there are too many variables that affect the readings.

\subsection{Modelling}

An energy model or simulation is required during the design phase of new or retrofitted buildings; specifically high performance buildings with the intention of meeting previously mentioned performance standards. Since VIPs are non-homogeneous in 3D, modelling them in 2D software such as THERM and WUFI require creating multiple cross sections. An area-weighted average would then be used to obtain an effective thermal resistance value. VIPs cause computational challenges due to the perimeter acting as a thermal bridge within the building envelope. 
Researchers have attempted to model the thermal bridging effects of VIPs and determine the significance of these factors. Boafo et al. [37] used a new modelling method to encompass the thermal bridge effects on the overall U-value of the building envelope, specifically retrofitted applications. They performed their simulations in a program named DesignBuilder [38], which uses EnergyPlus as the simulation engine. They utilized buildings with varying building envelope composition in Seoul, Korea as their case studies. In their simulations, they compared 10 systems retrofitted with VIPs and compared the heating season energy consumption (December 1st to January 31 st) when the thermal bridge of VIPs are included and excluded. The simulations resulted in a $3 \%$ to $10 \%$ difference in total energy consumption between the two methods, and listed the bridging percent factor between 2.30 and 3.85 for the main wall. They noted that the different layer compositions between the case studies affect the percent bridging factor, as well as the VIP geometry and properties (500 $\mathrm{mm}$ by $500 \mathrm{~mm}$ and $20 \mathrm{~mm}$ in thickness). It was also apparent that the thermal bridges have a significant effect on the energy consumption of buildings and needed to be taken into account as opposed to evaluating the envelope with the VIP's center of panel thermal conductivity, commonly provided by the manufacturer.

\subsection{Vacuum Insulation Properties and Alternatives}

Martin et al. [39] studied the thermal response of thermal bridges in both the dynamic and steady-state regimes through a guarded hot-box facility. Their motivation was the effect of thermal bridges on the building energy usage and determined the area of influence. The test results were compared to simulations results from FLUENT 6.2, which solves the energy equation for each time step. The dynamic test consisted of oscillating the simulated outdoor temperature to excite the surface. Martin et al. [40] furthered the dynamic analysis of thermal bridges, and a proposed 
methodology for finding an equivalent wall that can be used in the simulation. The method includes incorporating the heat flow and inertial effects of thermal bridging.

Brunner et al. [16] listed many alternatives to vacuum insulation during their review of VIPs. They proposed that vacuum insulation was not the only method to achieve a high level of thermal insulation. Gas-filled panels (GFPs) utilize a low conductive gas contained within a solid structure. Since the gas is at ambient pressure, the core material present in VIPs is no longer required. The GFPs contain a baffle structure inside to limit the amount of convective and radiative heat transfer within the panel. Similar to VIPs, GFPs use an envelope foil that suppresses gas diffusion since the performance is dependent on the specific gas content. Another high performance thermal insulation developed in the last ten years, listed by Brunner, are aerogels. Aerogels are typically composed of silica where the $\mathrm{SiO}_{2}$ chains with a large number of air filled pores. Due to the high porosity of the product, its inner structure provides many breaks causing a longer path for thermal transport causing a large thermal performance. Even though the aerogels have great thermal, physical, and acoustic properties, they have a low mechanical strength and require to be designed with other structural components.

Brunner et al. [41] also believe high performance insulation innovations would be based on the vacuum and gas panel technology but provide a robust design that currently limits the technology from being integrated to the building sector. Dynamic thermal insulation technology was included in the list of possible innovations. Since the thermal conductivity is directly related to the gas pressure, it was suggested to control the pressure within the VIPs to obtain a favorable thermal performance based on the heating and cooling requirements. Limitations included the large electric power requirement to venting and evacuating the large area, but could be a solution when 
high thermal resistance walls in buildings indirectly increase energy consumption in the cooling season.

This idea was further studied by Berge et al. [42] when they investigated controlling the internal pressure of a nano-porous material to vary the thermal conductivity. Through their laboratory measurements, a nearly linear relationship was found between pressure and thermal conductivity and the difference between the highest and lowest thermal conductivities was as high as a factor of 3: less than 2 for aerogels and around 3 for fumed silica. They implemented a variable insulation factor of 3 into the simulation of an office building located in Sweden and results showed that it could reduce the weighted energy demand by $20 \%$, where energy consumption was weighted 3:1 against cooling.

\subsection{Summary}

In summary, the introduction of voluntary codes has provided a pathway for super insulation, such as VIPs, to be integrated into the building envelope. While challenges of integration and evaluation are apparent, VIPs offer a solution with significant potential. The potential is the ability to create thin, high RSI-value building envelopes such that the walls do not compromise the thickness or floor space while providing energy savings. However, there are gaps that exist that still need to be filled.

Through in-situ measurements and infrared images, it is clear that significant changes in thermal resistance exist along the edges. This issue is uncommon for exterior insulation since it is typically a continuous layer of insulation. This property changes the analysis of the effective RSI-value of the building envelope caused by the increased number of cross section. The center of VIP shown to be some amount from the infrared images but the ability to efficiently evaluate the 
effective RSI-value is lacking. There is a lack of information in the literature about where the center of panel RSI-value is representative in the VIP with respect to the geometry.

This is problematic for evaluating VIPs in calculating the effective RSI-value of a building envelope. If the VIP is separated into two or more RSI-values, such that the edge (perimeter) and center have two distinct RSI-values, the coverage area is a variable that needs to be considered. The coverage area is an extra measurement required for effective RSI-value calculation, which is uncommon for building envelopes. When introducing this principle to two-dimensional heat transfer modelling, the evaluation process becomes difficult. The current practice requires a modelling geometry for each unique cross section. For example, if there are three different insulation levels at a single interface, at least three geometries need to be created. The computational difficulties may add time and effort that could discourage building designers or building modelers from considering certain materials. Furthermore, this issue is prevalent when VIPs are encased in other insulations as a means to achieve a thin, high RSI-value envelope and a protection mechanism for the VIPs. A methodology that is simple to follow and able to create a single profile geometry that represents a complex building envelope is missing in literature. 


\section{Chapter: Experimental Approach}

A study was conducted to evaluate the effective thermal resistance of full-scale building envelope assemblies and thermal resistances at various locations along the surface of VIPs were performed at steady-state with Carleton University's guarded hot-box. A vast amount of instrumentation, including thermocouples, heat flux plates, and power monitoring systems were used to evaluate the thermal properties. The guarded hot-box is an easily accessible apparatus used for rapid testing of designs at steady-state conditions in a laboratory setting. While other experimental systems, such as guarded hot plates, in-situ test facilities or an unguarded hot-box are able to evaluate the building envelope assemblies, the guarded hot-box is a highly instrumented experimental apparatus used to evaluate the effective RSI-value of building envelope assemblies at steady-state conditions with high accuracy.

In this chapter, the experimental apparatus and methodology is described. The function of the guarded hot box and its three chambers are explained. The data acquisition and control strategies, along with associated instrumentation used to evaluate the building assemblies, are listed. The guarded hot-box and thermocouple calibration procedures are presented. Finally, the evaluated VIP assemblies, which were studied, are described with the construction and instrumentation details.

\subsection{Guarded Hot-Box}

Since 2013, Carleton University has collaborated with NRCan-CanmetENERGY to study the performance of thin, high RSI-value building envelope components and complete assemblies, including but not limited to main walls, doors slabs, windows, and window to wall details. A guarded hot-box was designed, built and instrumented to conduct this work and the apparatus consists of three distinct chambers: metering, guard and climate chambers, schematically shown 
in Figure 3-1, and a photo of the apparatus place in Figure 3-2. Each chamber includes separate control and instrumentation systems to establish, monitor, and maintain steady-state conditions necessary to find the effective thermal resistance. The operation of the guarded hot-box is described and the parameters used to evaluate the RSI-value of the building envelope are listed in this section. Finally, the section is followed by a description and purpose of each chamber in the apparatus.

The apparatus creates a temperature difference across the specimen, fastened in the surround panel, by mimicking interior and exterior conditions. The climate chamber uses a refrigeration unit to reduce the temperature in the chamber, while the metering and guarded chambers use electric heaters to maintain a constant temperature slightly above room temperature. The metering and guarded chambers are set to the same temperature in order to force the heat flow from the metering chamber through the specimen. During the test period, the power consumed by the electrical heaters is accurately monitored and the heat transfer from the metering chamber through the specimen can be calculated. Using the measured surface temperatures, power consumption, and known metering area, the effective thermal resistance is calculated after steady-state conditions have been achieved.

The guarded hot-box was designed and built to evaluate the thermal resistance of envelope assemblies up to $2.1 \mathrm{~m}\left(7^{\prime}\right)$ by $2.4 \mathrm{~m}\left(8^{\prime}\right)$ in size. It contains a metered area of $1.5 \mathrm{~m}\left(5^{\prime}\right)$ by $1.2 \mathrm{~m}$ (4') at the center and provides an opportunity to test new and emerging building materials that show potential to improve building performance. The guarded hot-box was outfitted with a National Instruments data acquisition system to collect measurements from temperature and heat flux sensors and a Delta controller to control all heating and cooling equipment. 


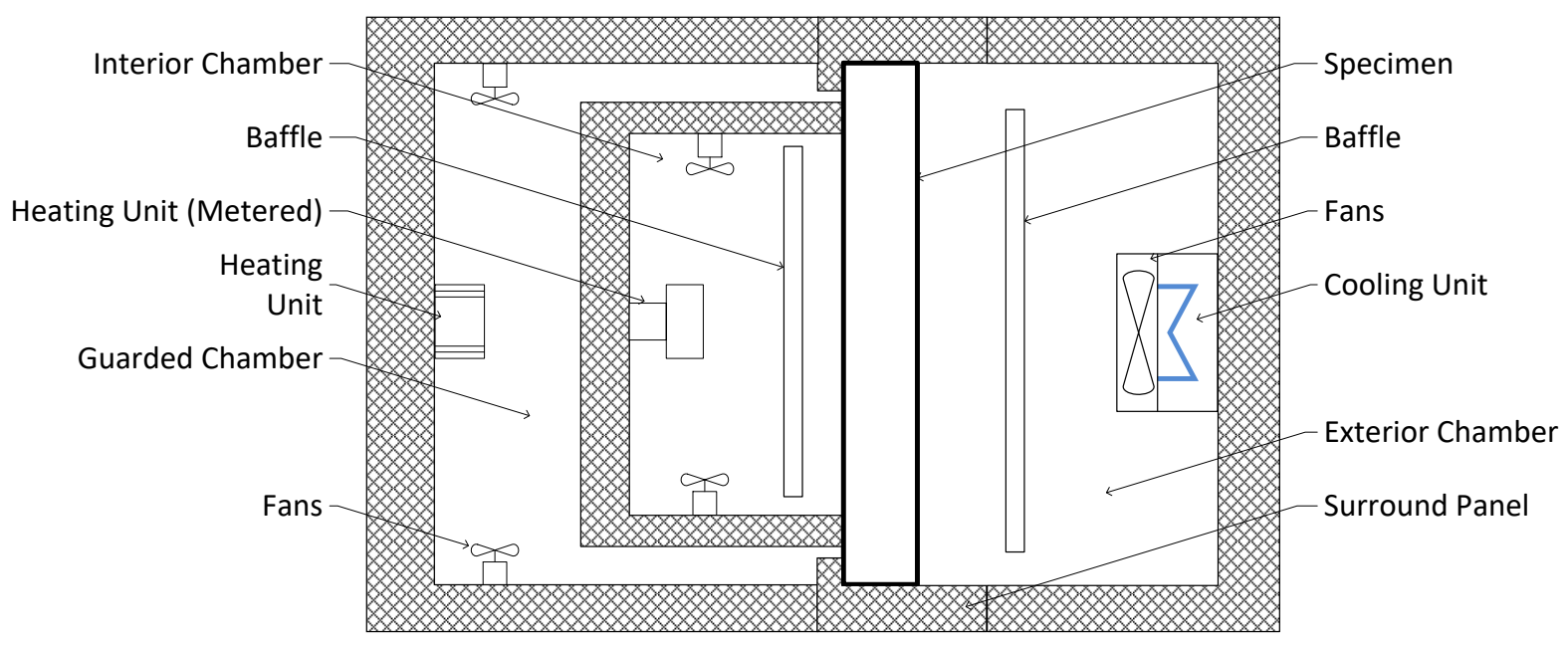

Figure 3-1: Simplified schematic of Carleton University guarded hot-box

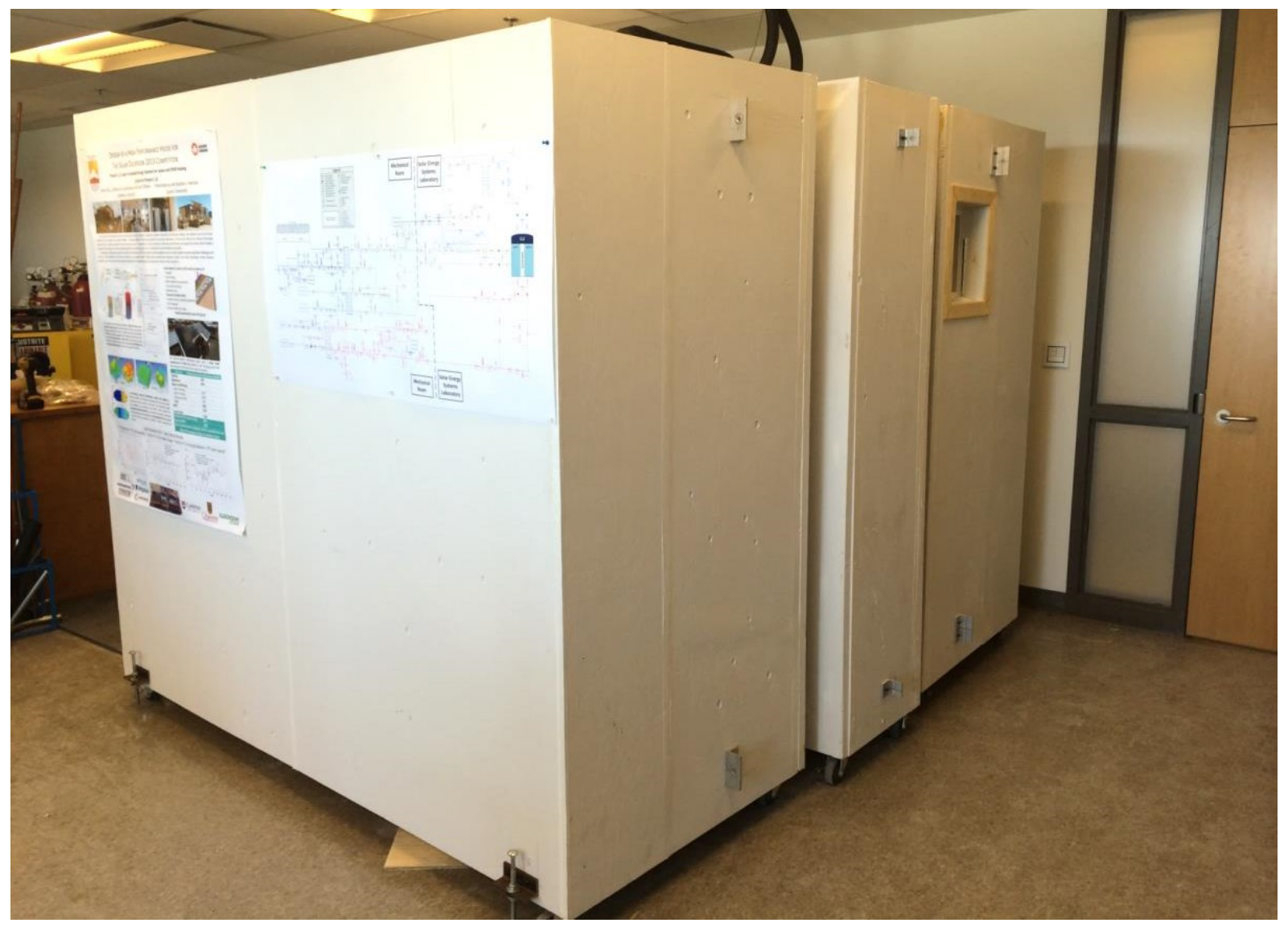

Figure 3-2: Photo of guarded hot-box located at Carleton University 
Steady-state test conditions inside the guarded hot-box were maintained when the three chambers were fastened and held together. In some cases, to meet the research objectives, it was necessary to capture infrared images of the interior surface to visualize the temperature gradients, thermal bridges, or cold spots that were present. In order to do this, the metering and guarded chambers were unfastened from the guarded hot-box apparatus, while the specimen and climate chamber remained connected. In this set-up, a handheld infrared camera was used to capture the surface gradients, shown schematically in Figure 3-3. The environment may affect the surface temperature and radiation energy, therefore the laboratory lights were turned off, and blinds were closed to limit any direct sunlight to the space or specimen. These conditions allowed the infrared images to capture the temperature gradients at the surface with limited effect from the environment.
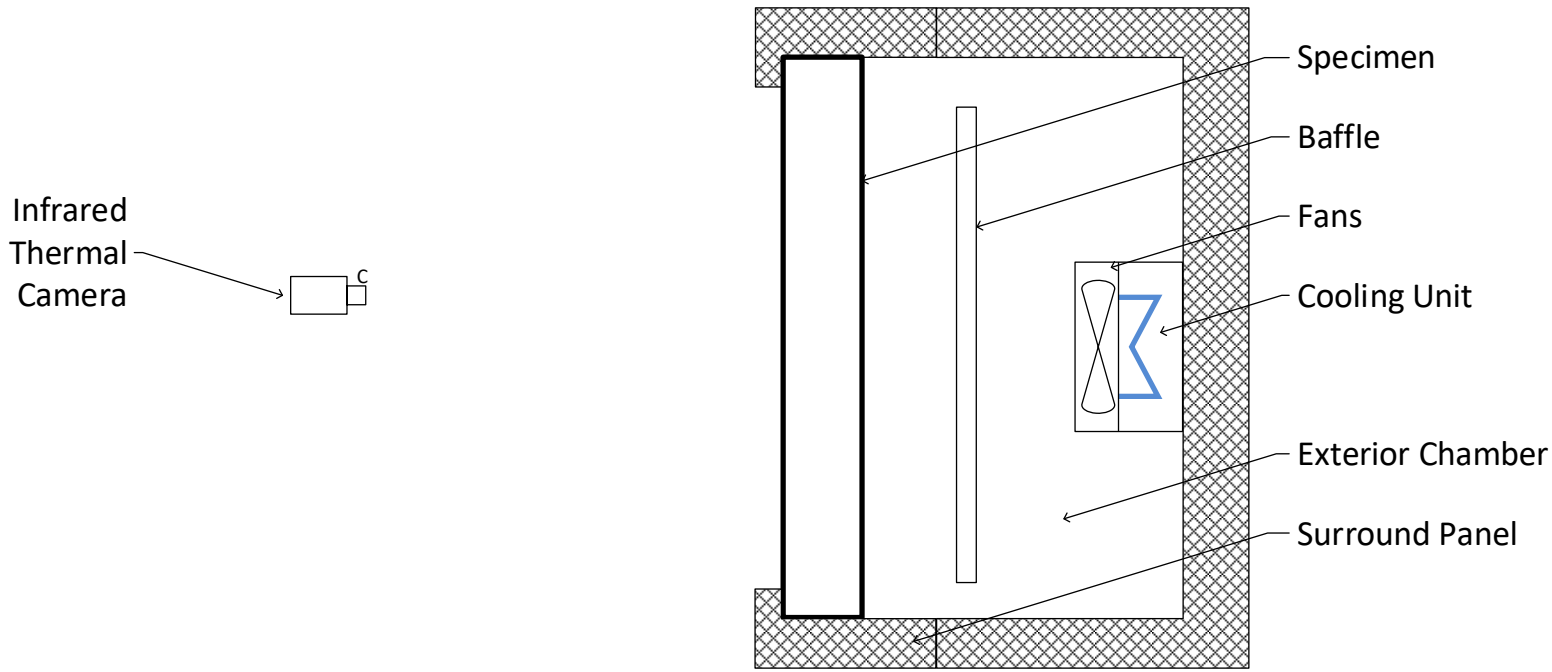

Figure 3-3: Schematic of guarded hot-box in secondary set-up when capturing infrared images

\subsubsection{Metering Chamber}

The metering chamber is the most vital chamber used in determining the RSI-value of wall assemblies in the guarded hot-box. The important properties that are relevant to evaluating 
building envelopes are the size of its opening, effective RSI-value of the metering chamber walls, the baffle, and instrumentation located inside. The metering chamber is a $1.5 \mathrm{~m}\left(5^{\prime}\right)$ wide by $1.2 \mathrm{~m}\left(4^{\prime}\right)$ tall by $0.5 \mathrm{~m}\left(1.5^{\prime}\right)$ deep box that simulates the indoor conditions. The chamber is fixed within the guarded chamber. A $25 \mathrm{~mm}$ (1") of XPS, equal to a RSI-value of $0.92 \mathrm{~m}^{2} \mathrm{~K} / \mathrm{W}$, is added to the metering chamber's walls. The insulation is added to limit the heat transfer from the metering chamber to the guarded chamber. A $500 \mathrm{~W}$ electric heater and a bank of fans operate simulatneously and are located behind a baffle in the chamber for heat and air dispersion. The conditions within the chamber are set to represent typical indoor temperature as well as air velocity consistent with natural convection. The temperature is set to slightly above the laboratory temperature for improved control since instant cooling of the chamber is not possible. A baffle was installed to moderate the air velocity in the chamber and limit the air currents to a natural convection state. The interior temperature and air currents align with the requirements outlined in ASTM C-1363.

The metering chamber is densely instrumented to evaluate and confirm steady-state operating conditions. The opening of the metering chamber is shown in Figure 3-4, where a variety of thermocouples and heat flux plates are visible. In total, 23 thermocouples, 3 heat flux plates, 1 relative humidity sensor, 1 pressure transducer, and a power monitoring system attached to heater are installed in the metering chamber. Of the 23 thermocouples, 12 are placed on the test specimen's warm surface with the use of aluminum foil tape, 6 thermocouples are suspended in the air curtain, which is the vertical plane $30 \mathrm{~cm}$ away from the surface, and the remaining 5 thermocouples are installed permanently on the surface of the metering chamber walls. The thermocouples on the specimen surface were arranged in a 4 by 3 equally spaced grid to ensure temperature uniformity, as prescribed in the ASTM C-1363 standard. 
The heat flux plates are attached to the surface of the test specimen, typically at points of interest such as highest and lowest thermal resistances and they are movable before any test. The power monitoring system measures the heat input to the chamber from the electrical heaters. The heat transfer between the guard and metering chamber boundary is measured with the temperature difference on either side on the boundary as well as the known surface area and effective RSI-value of the boundary.

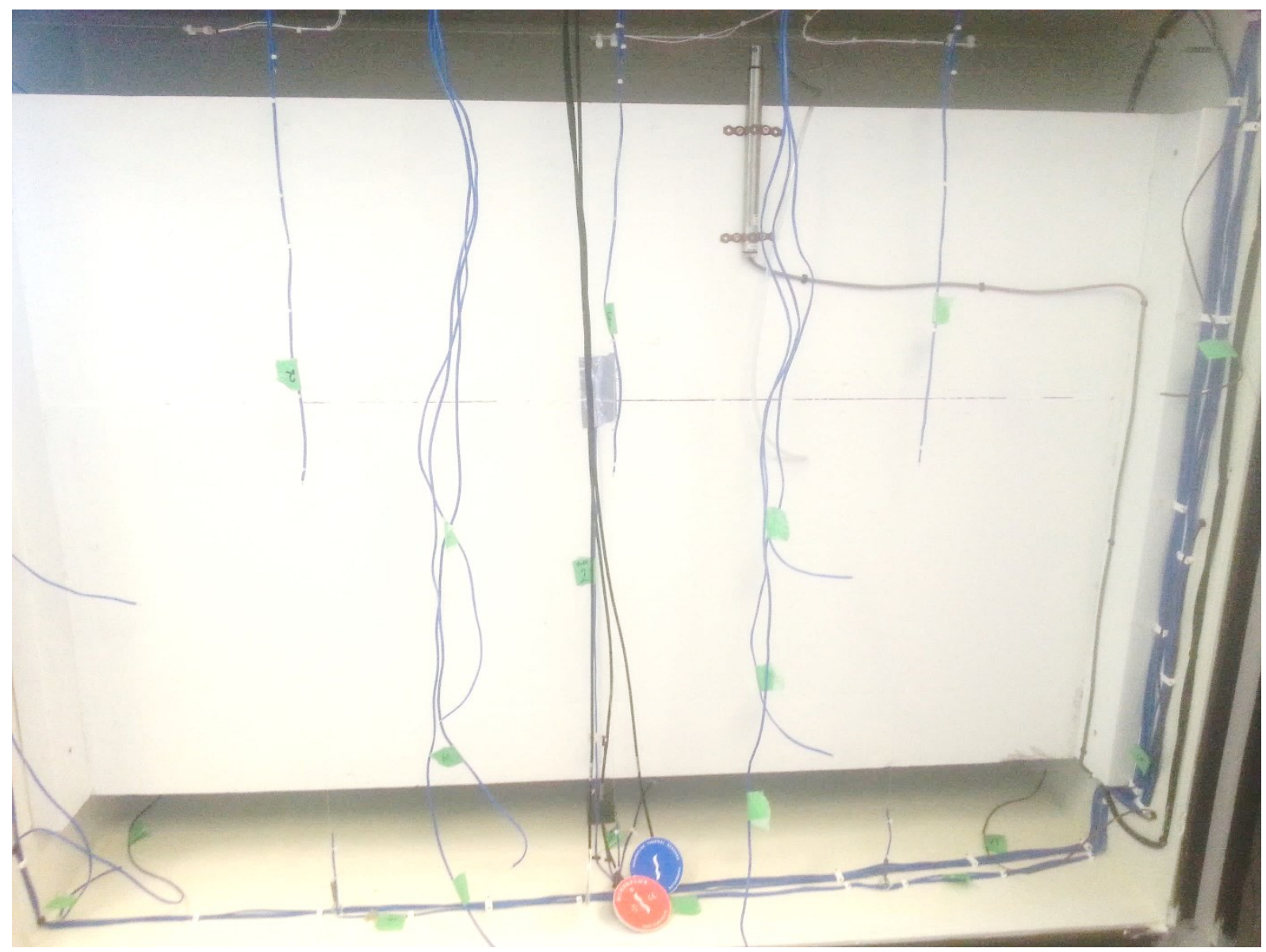

Figure 3-4: Metering chamber of the guarded hot-box

\subsubsection{Guard Chamber}

The guarded chamber effectively eliminates the heat exchange between the metering chamber and environment. The guarded hot-box acts as a buffer between the metering chamber 
and the environment and with the tightly controlled air temperature. A $2.3 \mathrm{~m}\left(7.5^{\prime}\right)$ by $2 \mathrm{~m}\left(6.6^{\prime}\right)$ and $1 \mathrm{~m}\left(3.3^{\prime}\right)$ deep box surrounds the metering chamber. The temperature conditions within the chamber are to be identical to the metering chamber such that the heat transfer through the metering chamber walls is minimal. The instrumentation within the guarded chamber is limited to thermocouples located on the metering chamber outer wall and along the surface of the wall assembly located outside of the metering area. The guarded chamber contains the gasket along the contacting surfaces to provide an airtight seal at the interface of each chamber. The airtight seal ensures that the air from the metering chambers is not mixed with air from the guarded chamber. The airtight seal allows energy provided to the metering chamber to flow through the specimen within the metering area and reduces any lateral heat flow.

\subsubsection{Climate Chamber}

The climate chamber simulates the exterior conditions during the test period and contains junctions for sensors embedded inside the assembly. The chamber contains permanent instrumentation that measure the surface temperatures inside and outside the metering area to ensure there is no lateral heat flow and to confirm the conditions remain at steady-state. The guarded hot-box requires all three chambers in order to successfully evaluate and maintain steady-state conditions during the steady-state test period.

The climate chamber is used to simulate the outdoor conditions required to create a sufficient temperature difference across the wall assembly. The chamber uses refrigeration equipment capable of providing $1500 \mathrm{~W}$ at $-35^{\circ} \mathrm{C}$. The heat rejection of the equipment integrates with the existing building and laboratory infrastructure. A large fan was installed to circulate the cold air and create a homogenous temperature throughout the chamber. A baffle was built and positioned in front of the large fan to limit the air speed at the surface of the wall assembly, thereby 
limiting the heat transfer by convection at the specimen's surface. The chamber measures $2.3 \mathrm{~m}$ $\left(7.5^{\prime}\right)$ by $2.0 \mathrm{~m}\left(6.6^{\prime}\right)$ and $1 \mathrm{~m}\left(3.3^{\prime}\right)$ deep in size, such that the perimeter is aligned with the perimeter of the guarded chamber.

The instrumentation installed within the chamber includes thermocouples, heat flux plates, and a relative humidity $(\mathrm{RH})$ sensor. In total, there are 20 thermocouples, 3 heat flux plates, a single RH sensor installed permanently, and the facility can embed 34 additional thermocouples and 16 additional heat flux plates within the building envelope. The installation of quick connect adapters on the wall side of the baffle, shown in Figure 3-5, was done in order to swap wall assemblies in and out of the guarded hot-box efficiently and shorten the necessary length of thermocouple wire. In this case, the sensor wiring does not have to return to the data acquisition unit during the specimen's installation. To connect the embedded thermocouples to the data acquisition unit, miniature thermocouple connectors from Omega were used also shown in Figure 3-5. They only require attaching a male adapter to the embedded sensor, which will mate with a female connector permanently installed in the climate chamber and wired to the data acquisition systems. For the heat flux plates, terminal blocks were added within the climate chamber to insert the wires of the embedded sensors into the screw terminals. The other end of the wire attached to the female connector and terminal blocks were wired directly to the data acquisition system. 


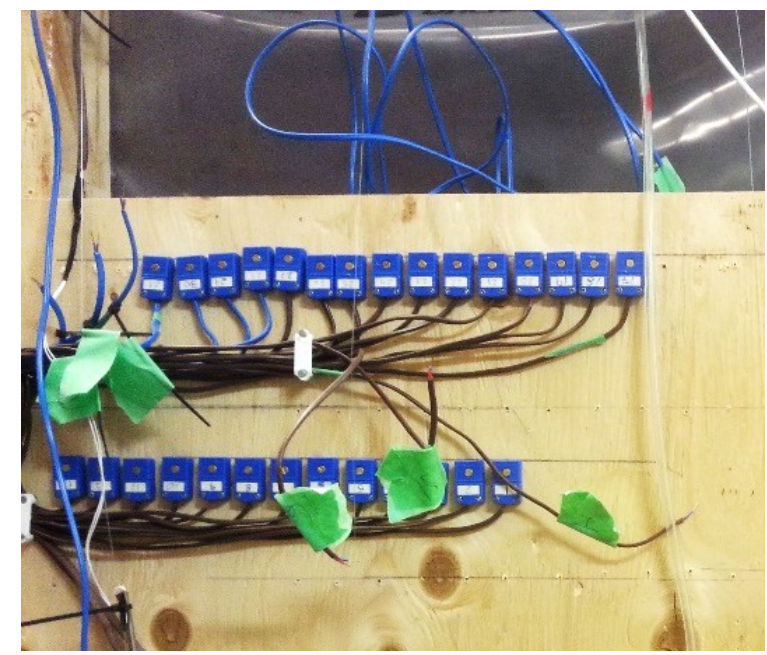

Figure 3-5: Quick connect thermocouples installed inside the climate chamber

\subsubsection{Data Acquisition and Control Systems}

A Delta controller and National Instruments CompactDAQ, shown in Figure 3-6 with individual photos in Figure 3-7 and Figure 3-8 respectively, were used to control the experimental conditions and acquire relevant measurements to determine the experimental RSI-values of building envelopes. The Delta controller monitors and controls the heating and cooling inside the three chambers. Delta offers continuity between the control systems used for the existing heat rejection infrastructure that the guarded hot-box uses during operation. The NI CompactDAQ (NI-cDAQ9188) is an 8-card data acquisition chassis connected to a computer through an Ethernet cable. 


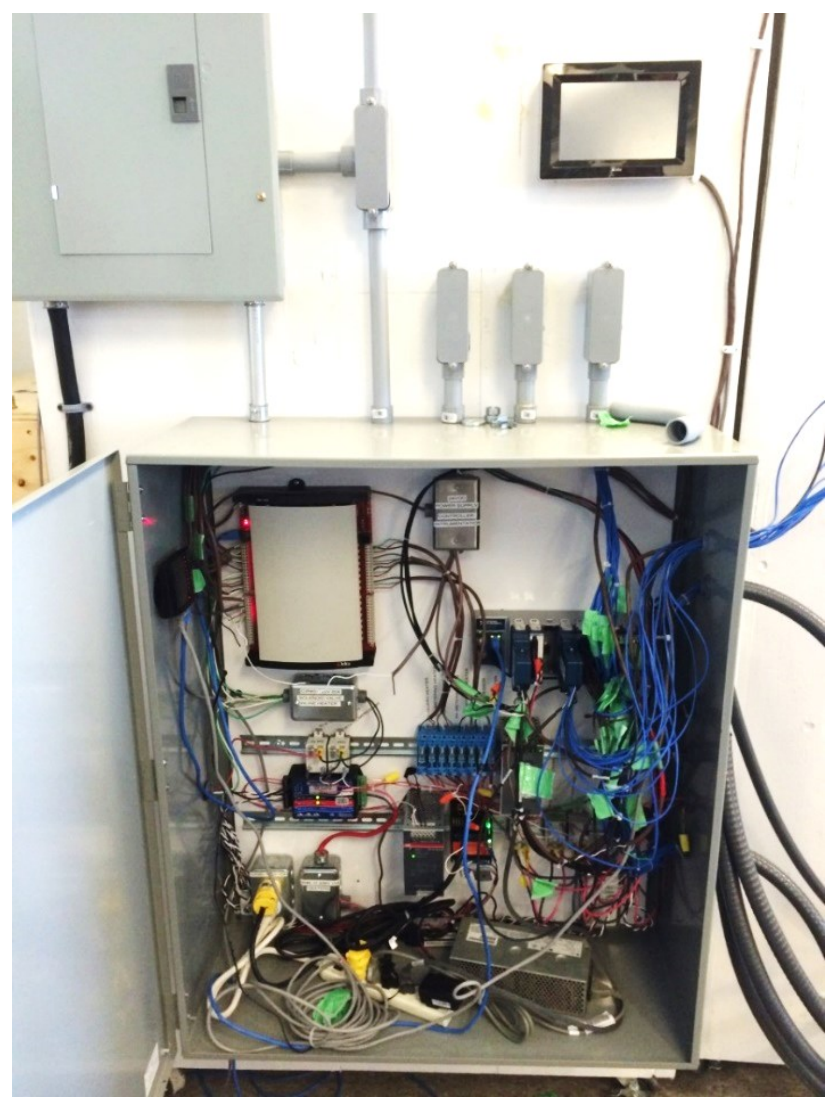

Figure 3-6: Photo of data acquisition and control systems used for guarded hot-box

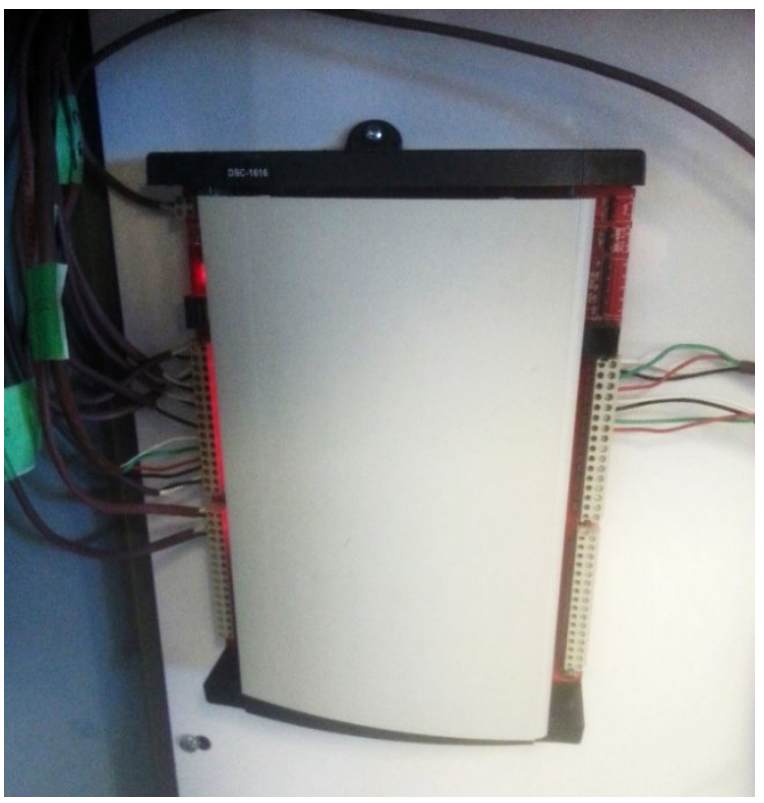

Figure 3-7: Photo of Delta controller integrated with the guarded hot-box 


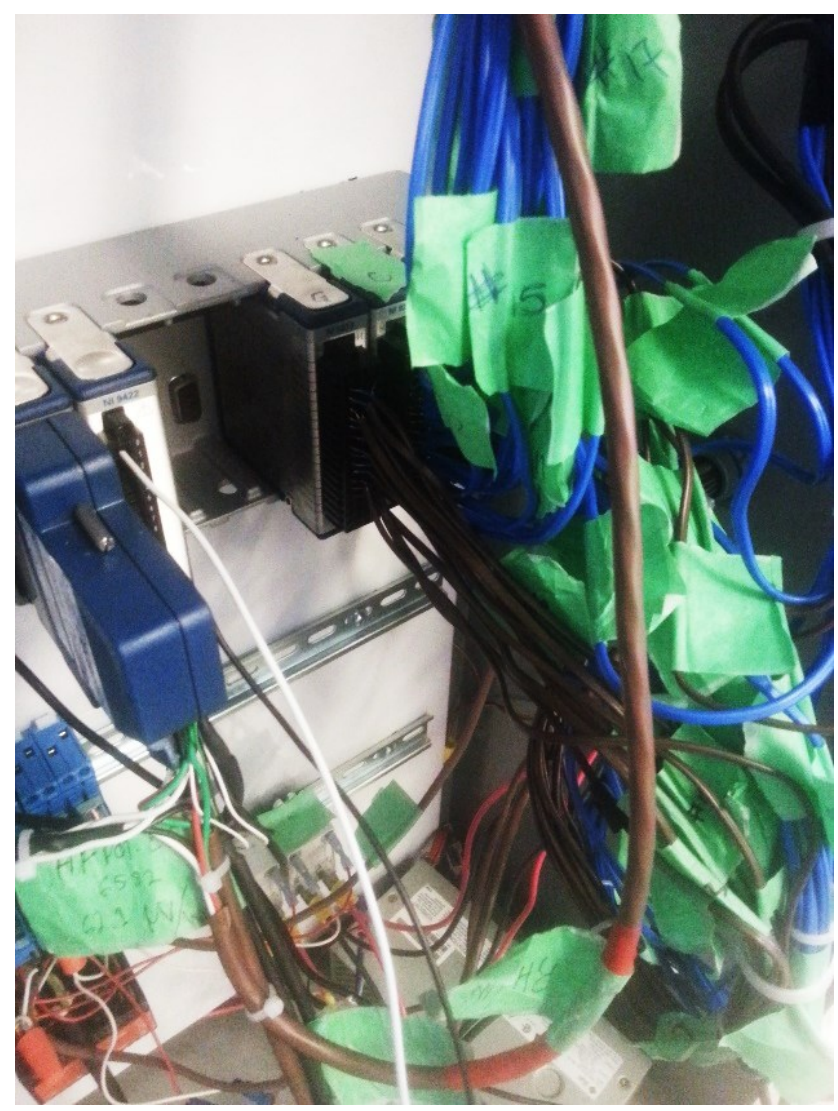

Figure 3-8: Data acquisition unit used for guarded hot-box with instrumentation connected

The computer is interfaced with the LabVIEW data acquisition software and can be programmed to acquire simultaneously and process data from the CompactDAQ. The data acquisition unit reads the sensor's signal through analog inputs of the card as a current, voltage or a pulse. Afterwards, LabVIEW scales the inputs to a temperature, heat flux through a calibrated sensitivity provided by the manufacturer (e.g., power monitoring, heat flux) or an equation developed through calibration procedures (e.g., thermocouples).

\subsubsection{Thermocouples}

In total, the guarded hot-box contains 59 thermocouples installed to measure temperatures at different locations within the chambers and wall assembly. All of the thermocouples used were calibrated before they were installed to an overall measurement accuracy of $\pm 0.48^{\circ} \mathrm{C}$, to reduce 
their uncertainty to meet the minimum ASTM requirement of an accuracy of $\pm 0.5^{\circ} \mathrm{C}$. Since the thermocouples are two wires of different materials, a voltage difference is created and read by the NI 9213 thermocouple input cards with onboard cold junction compensation. The thermocouples measure the air curtain temperature between the metering box and specimen, and the surface temperatures on either side of the specimen in the metering box and the climate box. At the air curtain (the space between the metering box and the specimen) a three by three matrix of thermocouples was placed to measure the air temperature slightly offset from the specimen. The thermocouples are suspended and organized in place using hooks, nylon string, and zip ties. On either side of the specimen, the thermocouples were arranged in a four by three matrix, to account for any temperature variations. Thermocouples are adhered to the surface with aluminum foil tape after the specimen is installed to eliminate any radiation and temperature variations from affecting thermocouple readings on the specimen. Remaining thermocouples were installed on both sides of the metering box walls, to measure the temperature difference at the guard box. The thermocouples provide an accurate point measurement of the surface or interface temperature through a voltage signal that is scaled to a temperature within LabVIEW.

\subsubsection{Power Monitoring}

A WattNode Pulse transducer was used to monitor the energy consumption of the heaters inside the guard and metering box. The transducer, in conjunction with a $15 \mathrm{~A}$ current transformer, emits a pulse after every $0.375 \mathrm{Wh}$ of electrical energy consumption. Since the metering box is at a constant temperature, the energy input to the heater is equal to the total heat losses from the metering chamber, when at steady-state. The heat transfer through the specimen is calculated by combining the known heat losses from calibration beforehand or measurement during the test 
period. The transducer is connected to the NI 9422 card, which then counts the number of pulses and provides a number to LabVIEW for further processing. The power monitoring is used to measure the energy added to the metering chamber, which when under steady-state is equal to the heat exchange through the specimen.

\subsubsection{Heat Flux Plates}

Hukseflux Heat Flux Plate (HFP01) sensors were used to measure heat fluxes, at locations of interest that can vary for each test. This instrument measured the heat flux is thermopile with the metered area guarded from the edges. The heat flux was measured by the small temperature difference across the thermopile and scaled to the calibrated sensitivity. They were installed into the apparatus with excess slack so that they can access any point on or within the test specimen of interest during testing. This feature is useful when testing different wall envelopes, and produces the specimen's temperature profile or accurately measures thermal bridging areas, such as wall studs, windows, doors or anywhere the thermal resistance is lower or higher than the effective value of the specimen. There are three plates installed in both metering and climate chamber and three additional junctions installed in the climate chamber for embedded sensors. These plates measured the heat flux with $\pm 5 \%$ accuracy and were scaled to $\mathrm{W} / \mathrm{m}^{2}$ in LabVIEW, based on a provided factory sensitivity with units of $\mu \mathrm{V} /\left(\mathrm{W} / \mathrm{m}^{2}\right)$. The heat flux measurements were essential in evaluating the range of RSI-values that existed within the specimen. The heat flux plates were used to evaluate thermal bridges within the specimens, such as the edges of VIPs or sections with a wood stud. 


\subsubsection{Thermistors}

The Delta controller operates with using resistance based temperature measurements, unlike the data acquisition equipment. Therefore, thermistors were suspended in each chamber for the temperature control inputs. $10 \mathrm{k} \Omega$ thermistors were added to the metering, guarded and climate chambers at various locations to measure the distribution in air temperature in the chamber. In total, five thermistors are located in the guarded chamber, three in the metering chamber, and three in the guarded chamber. Additional thermistors installed within the guarded chamber measure the expected variation in temperature between the top and bottom of the chamber. The thermistors contain larger in thermal mass and size. The large size was disadvantageous when embedding sensors at interfaces within the walls assemblies because a slim or small profile was desirable. However, this type of sensor was helpful for the controlling the steady-state conditions since the measured temperature contained less variation and was less prone to quick changes due to air currents in the respective chambers, allowing for better, more refined control with reduced cycling.

\subsubsection{Other Sensors}

The other sensors, such as relative humidity (RH) sensors, pressure transducers, and air velocity meters, installed within the apparatus were not utilized in the main analysis of the effective RSI-value of wall assemblies. The RH sensors were mounted in the metering and climate chambers, air velocity added to the climate chamber on the wall side of the baffle, as well as a pressure transducer to measure the pressure difference from the climate to metering chamber. These sensors measure the interior conditions to confirm that the guarded hot-box apparatus conforms to the operating conditions outlined in the ASTM C-1363 standard.

\subsection{Evaluation Methods}


The guarded hot-box has the ability to evaluate the effective RSI-value of a specimen and the individual RSI-value of a cross section or material within an assembly. Two evaluation methods exist for determining RSI-values at steady-state: the effective RSI-value (e.g., wall assembly) and a RSI-value of a cross section (e.g., center of VIP, stud, and cavity). The approaches use different instrumentation techniques to acquire the necessary data to calculate the result.

To measure the effective RSI-value of an assembly, the required measurements are the specimen surface temperatures in the metering and climate chambers, the amount of energy added to the system through the electric heaters, the elapsed time of the test period and the metering area of the apparatus. In addition to the values that are used to directly measure the effective RSI-value, the steady-state conditions need to be in place and follow ASTM C-1363.

After steady-state conditions are met for 5 consecutive measurement periods, outlined by ASTM C-1363, Equation 1 can be used to calculate the effective RSI-value, $R_{\text {eff }}$, in $\mathrm{m}^{2} \cdot \mathrm{K} / \mathrm{W}$

$$
R_{\mathrm{eff}}=\frac{\Delta T \cdot t \cdot A}{E}
$$

where $\Delta T$ is the temperature difference between the interior and exterior surfaces in ${ }^{\circ} \mathrm{C}, t$ is the elapsed time of the test period in hours, $A$ is the metering area in $\mathrm{m}^{2}$, and $E$ is the heat input to the metering chamber through the electrical heaters in watt-hours (Wh). Evaluating the effective RSI-value of the enclosure encompasses all thermal bridges located in the metering chamber. In addition, most new building codes require the effective RSI-value of the enclosure, as opposed to the RSI-value between the studs, also known as the nominal RSI-value.

When the RSI-value of an individual component of a building assembly is measured, a different approach is taken. There is no longer the need for power monitoring, and instead heat flux plates are utilized. They are installed at an interface that the thermal performance is desired, 
and the temperature sensors are added on the interior and exterior of the material. After steady-state conditions are reached similar to finding the effective RSI-value, a point RSI-value can be found using Equation 2; where $\Delta T$ is the temperature difference across the material in ${ }^{\circ} \mathrm{C}$, $q^{\prime \prime}$ is the heat flux measured in $\mathrm{W} / \mathrm{m}^{2}$ to find the thermal resistance, $R$ in $\mathrm{m}^{2} \cdot \mathrm{K} / \mathrm{W}$.

$$
R=\frac{\Delta T}{q^{\prime \prime}}
$$

\subsection{Calibration}

For the experimental set-up, two types of calibrations were performed. Thermocouples were evaluated using a measured cold junction compensation (CJC) temperature and an error of $\pm 0.48^{\circ} \mathrm{C}$ was calculated. Afterwards, the guarded hot-box was calibrated for the test procedure and operation. With a continuous assembly of rigid insulation under steady-state test conditions, the guarded hot-box experimental value was compared to the known RSI-value from the manufacturer. The accuracy of the guarded hot box was determined by comparing these two values.

\subsubsection{Thermocouples}

The calibration procedure for thermocouples involves comparing the values acquired through the data acquisition systems and NI 9213 card to a more accurate temperature sensor, outlined in detail by C. Baldwin [43]. In this case, a resistance temperature detector (RTD) probe was used. At least 3 thermocouples were made from each spool ( 5 spools in total) that was used to instrument the guarded hot-box for calibration. The thermocouples were attached to the RTD using plastic zip ties. During the calibration procedure, it was important to keep the tips of the thermocouples as close to the end of the RTD as possible since minor temperature variations could exist within the calibration bath. The probe was inserted into water-based temperature bath (Fluke 7008 micro-bath) and was placed in the center of the bath to ensure that the probe or any 
thermocouple does not touch the inside walls of the chamber. The RTD was connected to a digital multimeter and allowed the LabVIEW interface to read the resistance measurement and scale it to a temperature measurement from the manufacturer's equation, and read the thermocouples through the NI 9213 card as a voltage differential. Since the card uses a cold junction compensation (CJC) value to scale the temperature, it was important to read that value from the card as well.

The basis of the calibration procedure is setting and maintaining a constant temperature within the bath, taking multiple measurements RTD, thermocouples, and CJC simultaneously within a set period. The thermocouples and CJC compensation are compared to the RTD, which has a higher accuracy compared to the thermocouples. During operation, the operating parameters are the temperature resolution, settling time, measurement interval and measurement resolution set before any calibration procedure begins. The temperature calibration settings that were followed for the study included a temperature resolution of $2^{\circ} \mathrm{C}$, settling time of 10 minutes, a measurement interval of 5 minutes and a measurement resolution of 20 seconds. The temperature range was performed initially at $5^{\circ} \mathrm{C}$ to $95^{\circ} \mathrm{C}$ then $-5^{\circ} \mathrm{C}$ to $95^{\circ} \mathrm{C}$ afterwards to show that there is agreement and the process is repeatable. After the data was acquired, a unique sixth order polynomial relationship, in form of Equation 3, was created and a new error value was determined, where $V$ is the measured voltage in $\mathrm{mV}$ and $\mathrm{CJC}$ is the cold junction temperature measured by the data acquisition card.

$$
T=a^{*} V^{6}+b^{*} V^{5}+c^{*} V^{4}+d^{*} V^{3}+e^{*} V^{2}+f^{*} V^{1}+g^{*} V^{0}+\mathrm{CJC}
$$

Within LabVIEW, a generic thermocouple relationship exist for a given thermocouple type with the associated generic error. However, using this method of creating a new thermocouple relationship for each unique roll of thermocouples used for instrumenting the guarded hot-box, the error can be reduced to approximately $\pm 0.48^{\circ} \mathrm{C}$ in comparison to greater than $\pm 0.57^{\circ} \mathrm{C}$ for 
uncalibrated thermocouples. This value includes the errors from the thermocouple and data acquisition equipment. In Table 3-1, the coefficients of the fourth order polynomial were added for each roll used in the guarded hot-box. These relationships were implemented into the LabVIEW interface and the LabVIEW relationship for Type T thermocouples, developed by the National Institute of Standards and Technology [44] was no longer used.

Table 3-1: Coefficients for calibrated thermocouple equations for each different spool

\begin{tabular}{l|ccccccc}
\hline \multicolumn{1}{l|}{ TC Roll } & \multicolumn{7}{c}{ Coefficients } \\
\hline & $a$ & $b$ & $c$ & $d$ & $e$ & $f$ & $g$ \\
\cline { 2 - 8 } Roll 1 & 0.0034 & -0.0146 & -0.0149 & 0.1455 & -0.6962 & 24.67 & 0.1171 \\
Roll 2 & 0.0059 & -0.0246 & -0.0313 & 0.2509 & -0.8073 & 24.69 & 0.1012 \\
Roll 3 & 0.0012 & -0.1032 & -0.0018 & 0.1559 & 0.8044 & 24.65 & -0.0348 \\
Roll 4 & -0.0003 & 0.0067 & -0.0012 & 0.1413 & -0.7922 & 24.65 & -0.0708 \\
Roll 5 & 0.0178 & -0.0578 & -0.1193 & 0.5068 & -0.7246 & 23.95 & -0.0121 \\
\hline
\end{tabular}

\subsubsection{Guarded Hot-Box Calibration}

Prior to beginning the full wall assembly tests incorporating VIPs, a calibration of the test facility was performed by testing an assembly with a known RSI-value. This was done to determine the reliability of the results that the facility could produce. In order to create a wall with a known RSI-value, it was decided that the wall would also need to be completely homogeneous i.e., no frame components. Therefore, to obtain a specimen that had an RSI-value within the measurable range of the guarded hot-box facility, two $25.4 \mathrm{~mm}$ (1") sheets of XPS were installed. The calibration assembly provided an RSI-value of $1.76 \mathrm{~m}^{2} \mathrm{~K} / \mathrm{W}$ based on the material properties.

Before beginning data collection and analysis, the guarded hot-box was operational for 24 hours to reach steady state. Data was then collected and analyzed in five, 2-hour intervals, as required by ASTM standard C-1363-11. As seen below in Table 3-2, the average temperatures on either side of the specimen do not vary by more than $0.2^{\circ} \mathrm{C}$. In addition, the average temperature difference between the metering box and guard box remains within $0.1{ }^{\circ} \mathrm{C}$ and the heat input to the 
system remains within 3 Wh during each 2 hour time period. The heat input varied more than what was desired however, this was fixed by increasing the 2-hour averaging period. By analyzing the steady-state data, an effective RSI-value of $1.96 \pm 0.1 \mathrm{~m}^{2} \mathrm{~K} / \mathrm{W}$ was calculated and compared to a published value of $1.76 \mathrm{~m}^{2} \mathrm{~K} / \mathrm{W}$, using Equation 1. For a fair comparison between the two values, the surface film thermal resistances of $0.12 \mathrm{~m}^{2} \mathrm{~K} / \mathrm{W}$ and $0.03 \mathrm{~m}^{2} \mathrm{~K} / \mathrm{W}$ for the interior and exterior respectively [28] were subtracted from the experimental value. These values were considered since the published value for $50 \mathrm{~mm}$ of XPS did not encompass the convective resistance between the air within the chambers and surface of the assembly. Therefore, the effective RSI-value was calculated to be $1.81 \pm 0.1 \mathrm{~m}^{2} \mathrm{~K} / \mathrm{W}$ and the difference between the calibration and experimental values were within the range of error. As a result, the experimental facility was considered to be reliable. At this point, testing of new and emerging materials, wall assemblies, and construction methods could begin.

Table 3-2: Calibration data for 5 test periods of the guarded hot-box facility

\begin{tabular}{cccc}
\hline Climate $\left[{ }^{\circ} \mathrm{C}\right]$ & Metering $\left[{ }^{\circ} \mathrm{C}\right]$ & Guard to Meter Difference $\left[{ }^{\circ} \mathrm{C}\right]$ & Heat Input $[\mathrm{Wh}]$ \\
\hline-20.0 & 20.2 & -0.04 & 74.3 \\
-20.0 & 20.2 & -0.02 & 73.5 \\
-20.0 & 20.1 & -0.01 & 75.4 \\
-20.0 & 20.1 & -0.02 & 75.0 \\
-20.0 & 20.1 & -0.03 & 72.4 \\
\hline
\end{tabular}

\subsection{VIP Layer Testing}

While VIPs can offer a significant increase in thermal performance, the changes in thermal resistance throughout the panel are a main distinction in what differentiates the product from conventional building insulation materials. The existence of the non-homogeneous property was known, however the amount of thermal resistance lost compared to the center was not quantified. In addition, it was unknown at what the distance from the edge does the center of panel thermal 
resistance become homogeneous. The effect of offsetting or tiling these panels with a second layer was also unknown. Manufacturers use different surface areas and shapes as well as thickness, which could be used as an advantage by limiting the edge losses. If the VIPs are thin, a second layer could be implemented and remain near the same thickness of standard insulation boards, and tiled to provide a more homogeneous temperature distribution.

To assess these unknown parameters, it was proposed to test only the VIP layers of insulation prior to their installation into a full wall assembly. The size of panels used in this study were $457 \mathrm{~mm}(18$ ") by $558 \mathrm{~mm}(22 ")$ and $10 \mathrm{~mm}(0.4 ")$ in thickness with a published center of panel thermal resistance value of $4.0 \mathrm{~m}^{2} \mathrm{~K} / \mathrm{W}\left(24 \mathrm{ft} \cdot \mathrm{h}^{\circ} \mathrm{F} / \mathrm{BTU}\right)$ [45]. However, the panels were four years old and some degradation in thermal performance was expected. The VIPs needed to be mounted onto structural sheathing, therefore a $12.7 \mathrm{~mm}\left(0.5^{\prime \prime}\right)$ sheet of plywood was installed in the surround panel opening with Tyvek building wrap stapled on afterwards. After initial testing, it was observed that a sheet of thin insulation should be added on the climate chamber side. This was added to shield the heat flux plates from air currents. With the plywood and insulation added to the assembly, the measured heat flux variations were reduced while maintaining a significant temperature difference across the VIPs.

The edge effects of the VIPs in single and double layer assemblies were studied under steady-state conditions. The temperatures on either side of the VIPs were measured through embedded thermocouples. In addition, the infrared images of the interior surface were captured after the system reached steady-state. The metering and guarded chambers were disconnected from the facility to expose the interior surface to the laboratory. The surround panel and climate chamber remained fastened together along with continued cooling in the climate chamber. 
Infrared images are useful in determining failures or issues during testing that may not be apparent in the data or visually. Figure 3-9 and Figure 3-10 present infrared photos of two separate failed guarded hot-box tests, with the same arrangements of VIPs. The perimeter of the VIPs is outlined in the figures with other issues that needed installation of a new VIP or air baffle. In Figure 3-9, it is visible that there are multiple broken VIPs denoted by the lower temperature (blue) regions in the photo. Upon physical inspection, it was determined that the assembly contained compromised VIPs. However, they were able to maintain their rigidity. The second infrared photo showed the influence of the air from the refrigeration unit. The large blue area in the center indicates that the forced cold air from the refrigeration unit was causing a localized cold spot, compromising the steady-state conditions. The localized cold temperature is due to air leakage around or through the specimen or simply the fan blowing directly on the specimen surface. To eliminate these effects, a baffle was added to the climate chamber in-line with the fan to disperse the air at the cold surface. The assembly's perimeter and seams were also taped to seal the specimen further and avoid air leakage. Furthermore, the infrared images have an error that was outside the acceptable error bounds, and were used for quality control and visualization of results. 


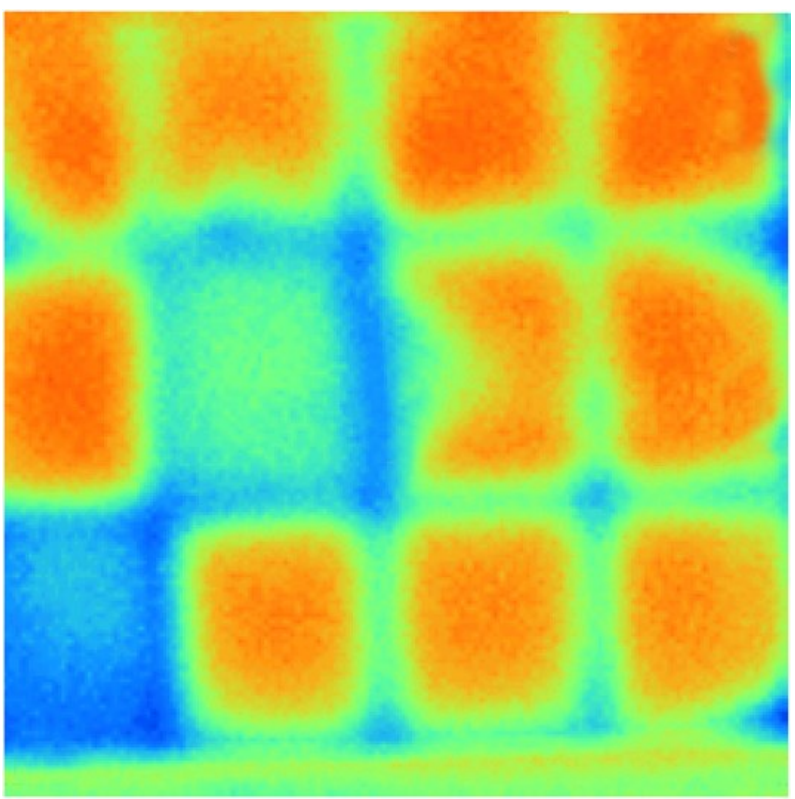

16.9

\section{0}

Figure 3-9: A compromised VIP layer featuring two broken VIPs

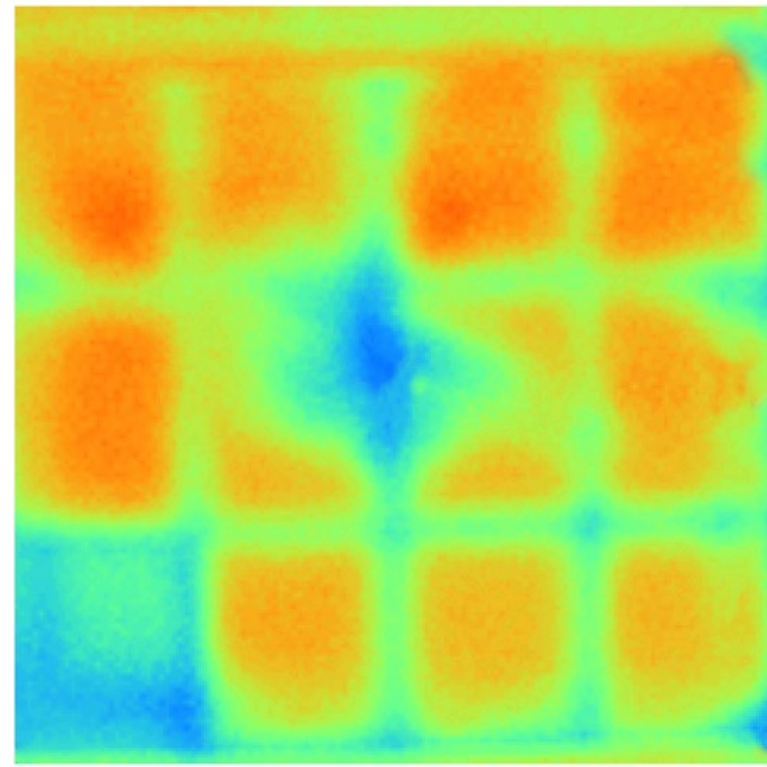

16.6

\begin{tabular}{l|l|l|}
13.0 & 24.0
\end{tabular}

Figure 3-10: Single layer VIP affected by fan blowing directly on specimen surface 


\subsubsection{Single Layer VIP}

A test was conducted to study the effect that the panel edges have on the overall thermal performance of a single VIP layer. The guarded hot-box was designed to accommodate 34 quick-connect thermocouples and 16 heat flux plates, in addition to the permanent sensors used to measure the effective thermal resistance. These embedded sensors, as well as infrared imaging, enabled the edge effects to be quantified around the VIPs. While embedding instrumentation within the VIPs may have disturbed the heat fluxes and temperatures within the wall assembly, the thermal resistances of the sensors are much smaller compared to the assemblies under evaluation.

Based on the VIP dimensions and the size of the surround panel, a 4 x 3 grid of VIPs placed as close to seam-to-seam as possible was made, shown in Figure 3-11. Note that a $140 \mathrm{~mm}(5.5$ ") gap between the VIP and surround panel wall was present on the left and right of the layer. This gap did not present a measurement problem in any of the analysis since this gap lies outside the metering area, and therefore the heat input from the metering chamber was transferred through VIP section. Another gap exists between the bottom of VIP layer and the surround panel, which lies outside the metering area and does not affect the results. Figure 3-11 shows a diagram of the VIP layer construction within the surround panel and metering area.

The anticipated result was that the heat flow and temperature distribution of each VIP would be the same due to symmetry. As such, a single VIP was heavily instrumented using a 5 x 6 grid of thermocouples locasted on warm side as shown in Figure 3-12. The denoted numbers with circles represent thermocouples, and the "HF" is where heat flux plates were embedded. One heat flux plate was placed on the XPS and VIP interface at each point where a change in thermal resistance was expected to take place, i.e., center, seam, and corner of the panel. 

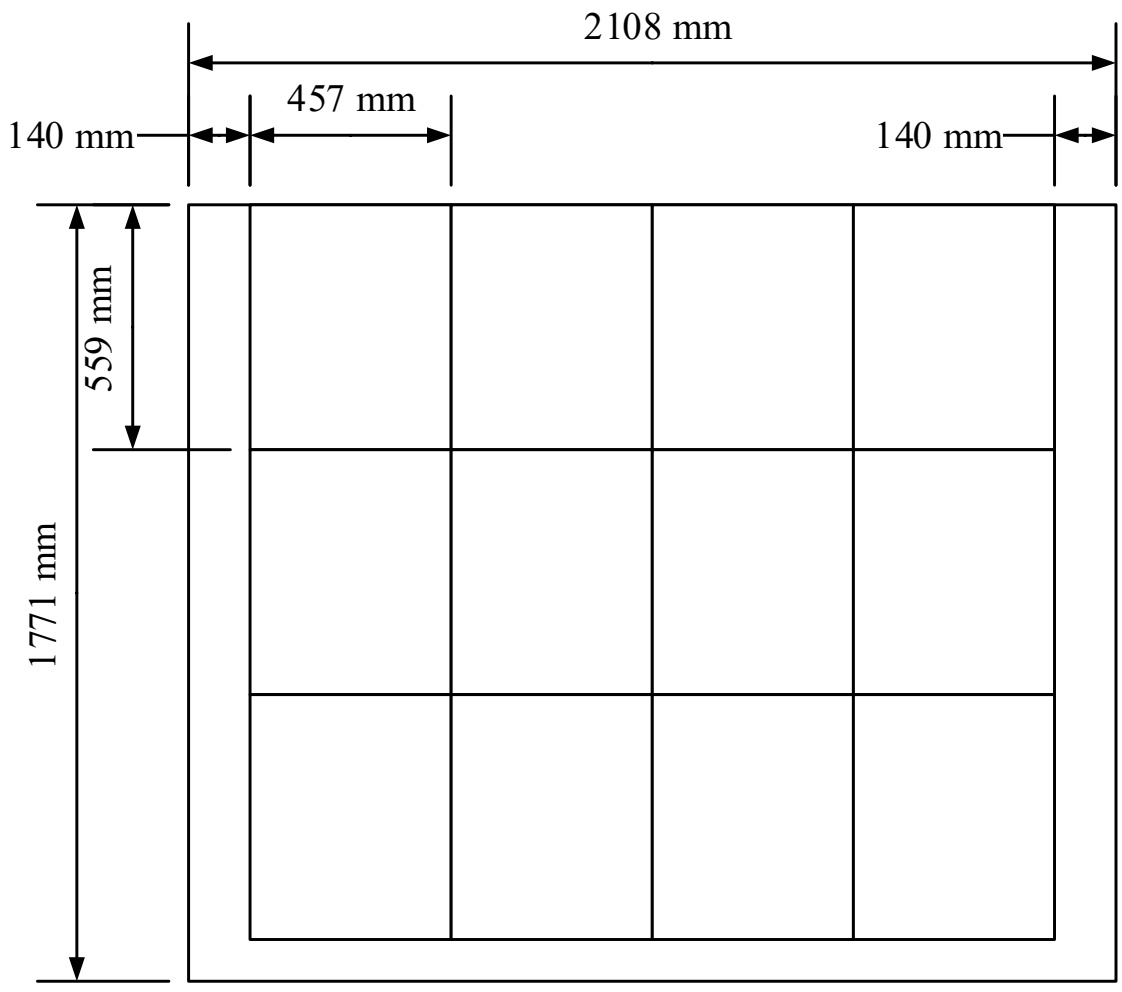

Figure 3-11: Single layer VIP layout within surround panel with dimensions

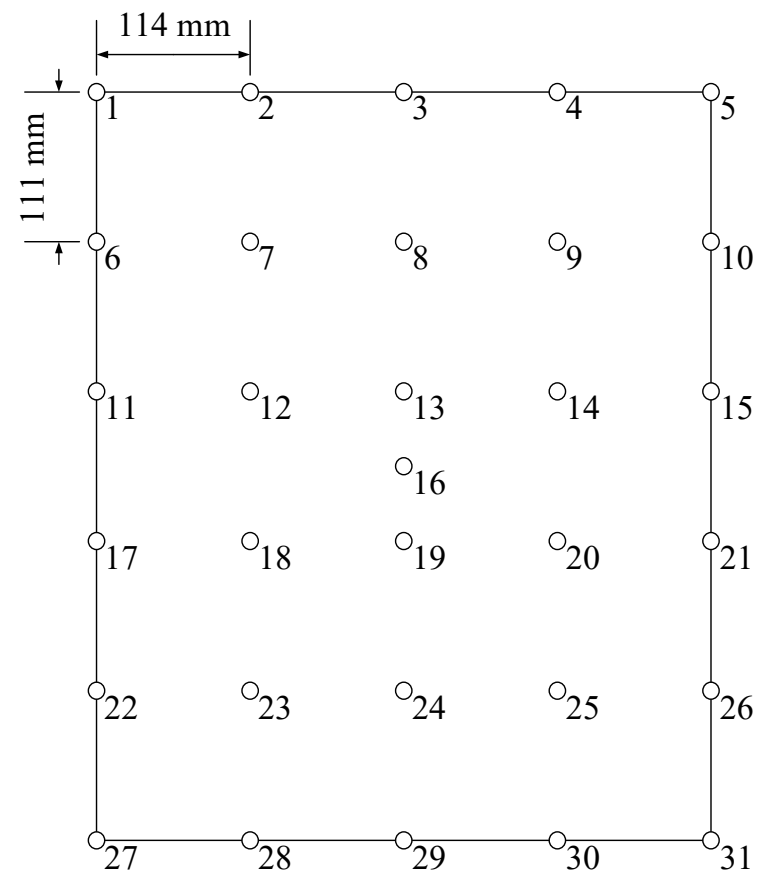

Figure 3-12: Labeled diagram of embedded instrumentation layout on the warm side of a single VIP 
This instrumentation approach yielded a steady temperature gradient on the warm side of the VIP and allowed the distance from the edge where the temperature becomes consistent with the center of the panel to be determined. The various heat fluxes and thermal resistance at the center, seam, and corner of a single panel were measured as well. This information and values are beneficial when effectively model a VIP effectively and finding the effective thermal resistance of the main wall assembly. The infrared photos captured after each steady-state test verified that there were no compromised panels in the assembly and allowed for another temperature gradient analysis at the wall surface.

\subsubsection{Double Layer VIP}

After analyzing the single layer of VIPs, a second layer of VIPs was added to study the effect on the overall thermal performance and temperature distribution. The evaluation principles used are the same as the single layer VIP procedure. Embedded thermocouples and heat flux plates were used to gain experimental data and using thermal imaging was used to analyze the temperature distribution at the interface. The same types of VIPs were used to build the second layer and tiled into three different types of configurations through offsetting the center of panels from the second layer to align with the center, seam, or corner of the first layer panels. Figure 3-13 and Figure 3-14 present the drawings of the three configurations, where the dashed red lines represent the second layer panels, and the solid black lines are the first layer panels. 

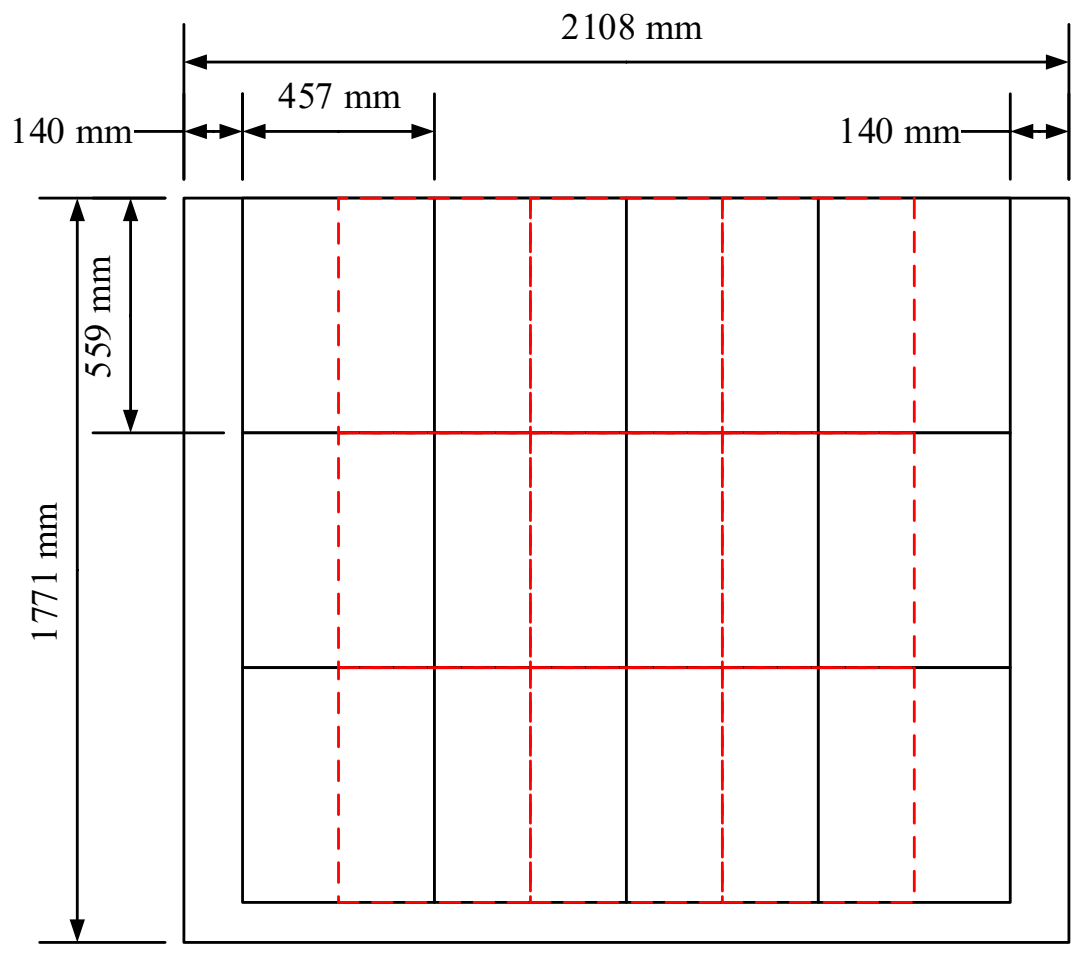

Figure 3-13: VIP layout of second layer offset to center-seam orientation
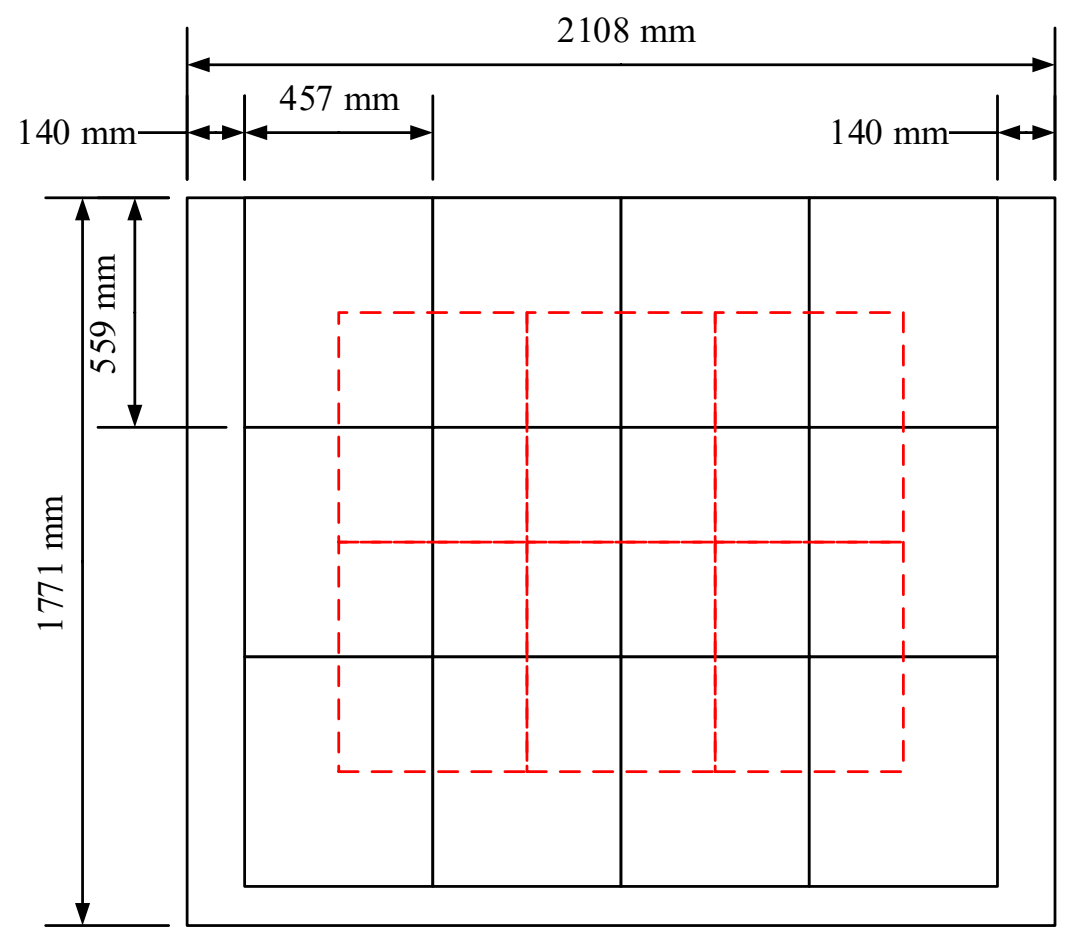

Figure 3-14: VIP layout of second layer offset to center-corner orientation 
The heat flux plates remained in the same location as the single layer test, but on the VIP-VIP interface, since the heat flux at each unique point was to be monitored, and these values will vary as the second layer varies. However, the thermocouples were organized so that 17 would be present at the VIP-plywood interface, and the remaining 17 thermocouples were located on the VIP-VIP interface. This layout allowed a temperature profile through the assembly to be measured as well as the temperature change caused by different configurations to be measured.

\subsection{Composite Insulation Panels}

After assessing the performance of single and double layer VIP panel sections, the effectiveness and implementation of incorporating VIPs into prefabricated XPS composite panels were studied. This design offers a solution to the fragility and constructability problems that are inhibiting the use of VIPs into residential building envelope construction. In essence, these composite panels need to be safely fabricated with strict quality control measures, and locations for fasteners clearly labeled for the easy installation at the construction site. This method mitigates the risk of puncture during manufacturing of the composite panels and construction of the home, while also providing the building with a thin, high performance enclosure. The purpose of encasing the VIPs in another insulating material is to mitigate the thermal bridge that exists along the fastening areas and could reduce the cost on a per square foot area with a proper commercial process. The size of the composite panels was designed to be $2.4 \mathrm{~m}$ (8') by $0.6 \mathrm{~m}\left(2^{\prime}\right)$ and $50 \mathrm{~mm}(2$ ') in thickness. The composite panels were constructed using $25 \mathrm{~mm}(1$ (') thick VIPs and XPS spacers in between two $12 \mathrm{~mm}\left(0.5^{\prime \prime}\right)$ thick, full-size XPS sheets. One design iteration presented in Figure 3-15 has the top XPS sheet removed such that the VIP and XPS spacers are visible. 


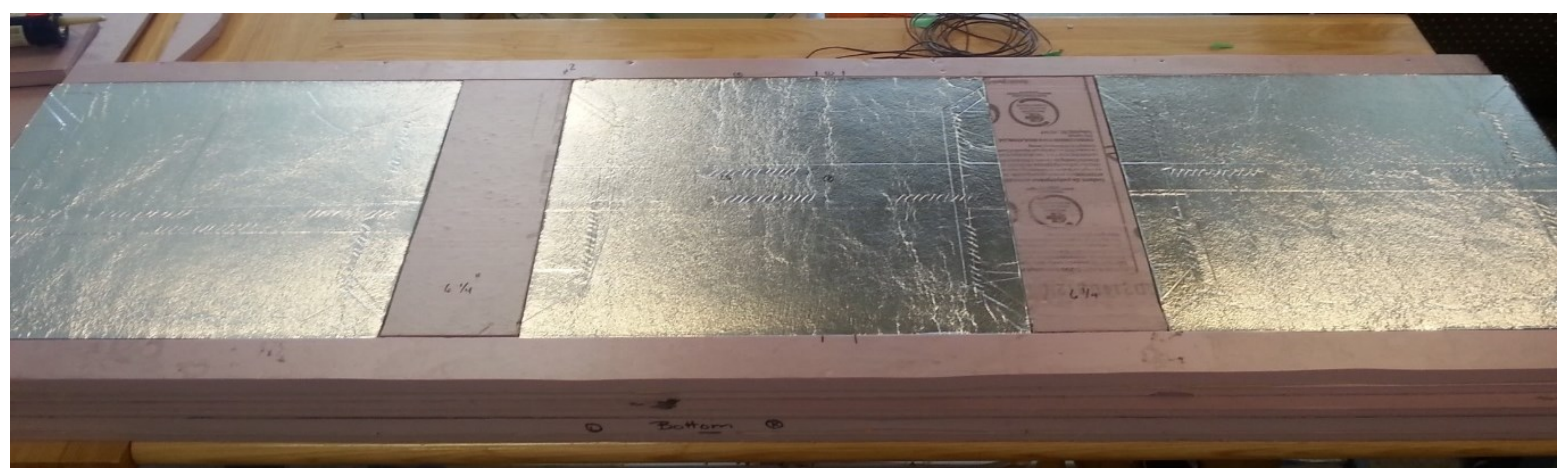

Figure 3-15: Construction of a composite panel with VIPs encased in XPS with top sheet removed

\subsubsection{Wall Assembly Composition}

Contrary to evaluating the single and double layer VIP layouts, it was desired to evaluate the entire wall composition because of the increased thermal breaks caused by the XPS spacers and edges of VIPs within the assembly. Due to a large number of thermal breaks and the desire to test a full main wall, the stud spacing was also varied to determine the thermal and design effects they would impose.

The composition of the wall assemblies used in the study resembles code-built wall assemblies in Ontario, Canada. The walls were built using 2x4 lumber on either $406 \mathrm{~mm}$ (16") or 305 (12”) spacing. Batt insulation was added between the studs with gypsum board and plywood sheathing. Finally, the composite insulation panel was added to the plywood sheathing and vinyl siding added to the exterior. The design also required a minimum size of XPS spacers for the mechanical fasteners. The wall composition is illustrated with a horizontal cross section drawing of the main wall with the building materials labeled in Figure 3-16. 


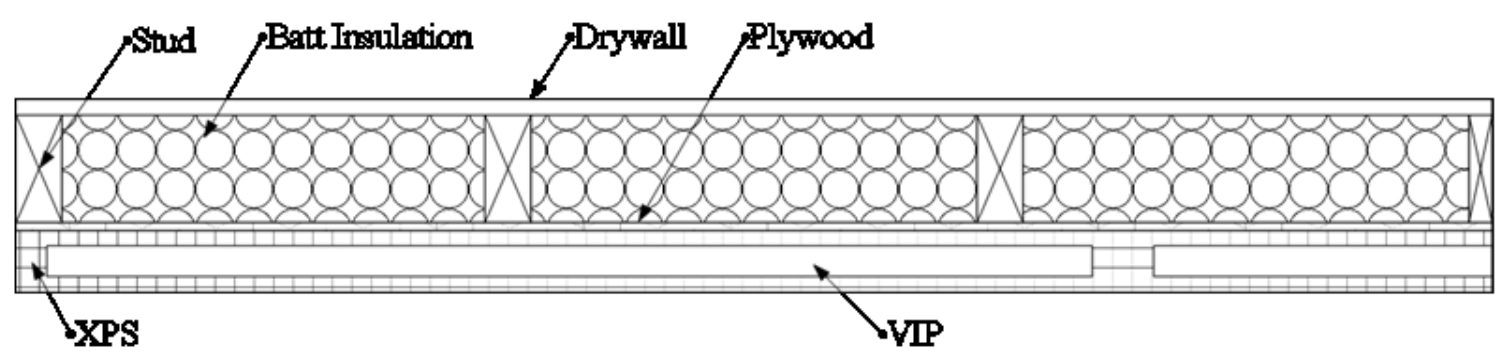

Figure 3-16: Example composition of wall assembly utilizing composite panels with VIPs.

\subsubsection{Five Integrated Designs}

Five different composite panel arrangements were built, metered, and tested to evaluate the thermal performance of these wall assemblies. The wall assemblies were also tested to assess the feasibility of composition insulation panels with VIPs as a solution to create thin, high performance envelopes for residential homes. Four different dimensions of the composite panels were adjusted: XPS dimensions, VIP dimensions, wood stud spacing and VIP manufacturer. The XPS dimensions were varied to determine the required safety distance for mechanical fasteners in a laboratory setting. The changes in VIP and spacing sizes enabled the study of the thermal performance as a function of VIP and XPS coverage. In Figure 3-17, the drawing shows a generic layout of a wall assembly where the labels are associated with the dimensions listed in Table 3-3. 


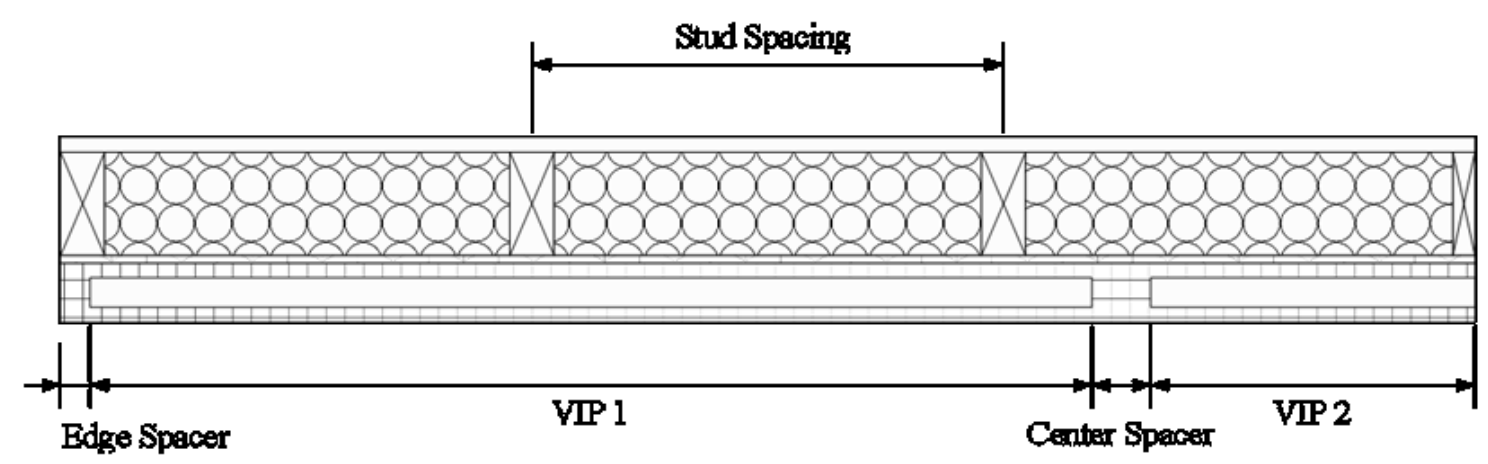

Figure 3-17: Legend for dimensions of 5 wall assembly designs provided in Table 3-3

Table 3-3: Horizontal dimensions to the center of wall for 5 composite wall assembly designs evaluated

\begin{tabular}{cccccc} 
Test & $\begin{array}{c}\text { Stud spacing, } \\
\text { mm (in) }\end{array}$ & $\begin{array}{c}\text { Edge spacer, } \\
\text { mm (in) }\end{array}$ & $\begin{array}{c}\text { Centre spacer, } \\
\text { mm (in) }\end{array}$ & $\begin{array}{c}\text { VIP 1, } \\
\text { mm (in) }\end{array}$ & $\begin{array}{c}\text { VIP 2, } \\
\text { mm (in) }\end{array}$ \\
\hline 1 & $305(12)$ & $25(1)$ & $51(2)$ & $559(22)$ & $559(22)$ \\
2 & $406(16)$ & $25(1)$ & $51(2)$ & $864(34)$ & $279(11)$ \\
3 & $406(16)$ & $0(0)$ & $25(1)$ & $597(23.5)$ & $597(23.5)$ \\
4 & $406(16)$ & $25(1)$ & $0(0)$ & $597(23.5)$ & $597(23.5)$ \\
5 & $406(16)$ & $25(1)$ & $51(2)$ & $559(22)$ & $559(22)$
\end{tabular}

In total, VIPs were purchased from two different manufacturers and incorporated within the assemblies. The assemblies contained two different types of wood stud frames with the same cavity insulation. The walls were assembled using $38 \mathrm{~mm}$ by $89 \mathrm{~mm}(2 \times 4$ lumber) wood studs with both $305 \mathrm{~mm}(12 ")$ and $406 \mathrm{~mm}\left(16^{\prime \prime}\right)$ on-center spacing to represent a common home in Ontario. RSI-2.4 (R-14) batt insulation was used to fill the cavities between the wood studs. This resembles current code-built building envelope construction in Ontario.

The VIPs from Manufacturer A were two different sizes, $863 \mathrm{~mm}$ (34”) by $559 \mathrm{~mm}$ (22”) and $559 \mathrm{~mm}$ (22") by $559 \mathrm{~mm}$ (22"). Meanwhile, the panels from Manufacturer B was $500 \mathrm{~mm}$ (19 3/8") by $600 \mathrm{~mm}(23$ 1/2") panels and also had different effective thermal conductivity. The different sizes of VIPs are used to obtain different ratios of VIP coverage area, where a larger value provides a larger effective thermal resistance, but the minimum size of XPS spacers also affects the number of VIPs within the composite panel. 
The required results from the test specimens outside of the effective thermal resistance include the temperature at each interface of the assembly along the four different cross section, the heat flux at different cross sections (e.g., XPS-stud, VIP-stud, XPS-cavity, and VIP-cavity) and finally the temperature distribution from the edge to the center of VIP. These results were used to evaluate the overall performance, compare the related results to a simulated model from THERM, and compare the VIP performance from each manufacturer. The instrumentation locations in section view for Tests 1-5, are shown in Appendix 1, where the red lettering is where the location of the embedded temperature sensors at each interface throughout the entire wall assembly are

located and the "HF" label represents the location of the heat flux plates. The amount of instrumentation embedded with the composite panels and wall frame, in addition to the permanent sensors in the guarded hot-box facility, provided many valuable insights. After the study, the thermal performance of the composite panel was evaluated with the guarded hot-box and the ease of installation was assessed from the construction and installation in a laboratory setting.

\subsection{Summary}

This chapter included a detailed description of the guarded hot-box apparatus, instrumentation and how it is used to measure the effective RSI-value of building envelope assemblies. A description of the calibration process for the guarded hot-box and thermocouples were provided with resulting uncertainties. Instrumentation and schematic drawings of the VIP and composite insulation panel testing were provided as well as challenges presented during the initial testing. The next chapter will cover the modelling approach used to evaluate the building envelopes incorporating VIPs using THERM. 


\section{Chapter: Modelling Approach}

The modelling approach for this study was to determine the effectiveness of reducing the complexity of the geometries required to evaluate a building envelope assembly. The steady-state experimental results to verify the modelling results produced using two-dimensional heat transfer software. Before new and emerging materials can make it to market, the material properties need to be determined, and proof of concept designs should be created and evaluated. While experimental research is an essential aspect of these evaluations and product development, obtaining the performance or optimization of wall assembly designs can be performed faster through modelling, provided the necessary material properties are available. Building envelope modelling is performed to evaluate the heat, air, and moisture (HAM) transfer through building envelopes, which yields results related to the long-term performance, durability and the effectiveness of different envelope designs.

VIPs offer a unique problem for heat transfer modelling of building envelopes due to their non-homogeneous nature. The non-homogenous edges are an unconsidered effect during present modelling techniques for building assemblies using VIPs and leads to an inaccurate effective thermal resistance. The center of panel RSI-value provided by the manufacturer may not be comparable to the actual performance when the edge are incorporated. In some cases, the center of panel RSI-value is provided by the manufacturer, while other times they provide an effective RSI-value for the panel. These terms can be misleading and may not provide the information that is required. For example, when evaluating the isothermal lines or the change in heat flow through the assembly, it would be more beneficial to use the center of panel RSI-value. However, when the evaluating the effective RSI-value of a building envelope, the effective RSI-value of the VIP is desired. The initial steps in this project were to evaluate experimentally the edge effects that 
VIPs contain, regarding maximum and minimum heat flows through the assemblies and then utilize the results and findings to report a better method to represent VIP assemblies.

\subsection{THERM}

A common tool used to evaluate and simulate the 2-D heat transfer through a wall assembly is the THERM finite element heat transfer software. The program was developed by Lawrence Berkley National Laboratory (LBLN) and is used for the simulation of building envelope penetrations such as windows, doors, and roofs among others. This software can be used to evaluate whole wall assemblies and has a detailed library that provides thermal properties of many building materials including various types of structural framing and insulations. The finite element method approach that the program uses allows the user to model the geometries of building products and materials. The software evaluates the energy efficiency of the system and local temperature variations that could directly affect other parameters such as of moisture damage and condensation formation.

THERM contains three main features to the software: graphic interface, heat transfer analysis and the results display. In the graphic interface, the user can draw the cross section of interest and assign the material properties. THERM allows the user to trace an imported computer aided drawing of a building component, or manually draw the geometry using the THERM dimensioning tools. Afterwards, each small component in the program needs to be assigned with a thermal material property and boundary conditions must be assigned to the external edges of the cross section. These properties may be assigned through THERM's library of materials or a new material may be created, which is the case for VIPs.

After the properties and boundary conditions are properly set, the heat transfer component begins. The program uses two-dimensional conduction analysis with a finite element method. The 
model can solve the problems associated with thermal bridging and complex geometries such as window frame or headers. The program auto-generates a non-overlapping mesh through the Quadtree method, essentially an algorithm to partition a space into four quadrants [46]. The quadrants are continuous broken into four sub-quadrants until they are considered balanced, which is related to the size of the node (element) and its adjacent counterpart. There have also been studies that put other restrictions on the balanced nodes, for example the mesh density, the size of an adjacent node or its proximity to a boundary condition [46]. After the mesh is balanced, THERM performs the analysis with an error estimation and returns the converged solution in the results component.

The results component provides the user multiple values and visualizations of the building component after simulation. THERM outputs the U-factors (inverse RSI-values), isotherms through the component, heat flux vector plots, and local temperatures. The U-factors or RSI-value is the thermal performance of the building component provided in a single value, shown in Figure 4-1. The isotherms are lines of constant temperature through the component. The lines are provided as individual isotherms or as an infrared image, with coloured pixels scaled to temperature. The heat flux vectors are arrows where the increased arrow density represents a large heat flux through the component. Similar to the isotherms, they can be outputted with the arrows or a colour-coded image. The results allow the user a variety of resources to determine the energy efficiency or temperature distribution, which relates to condensation and integrity, of a building component. 


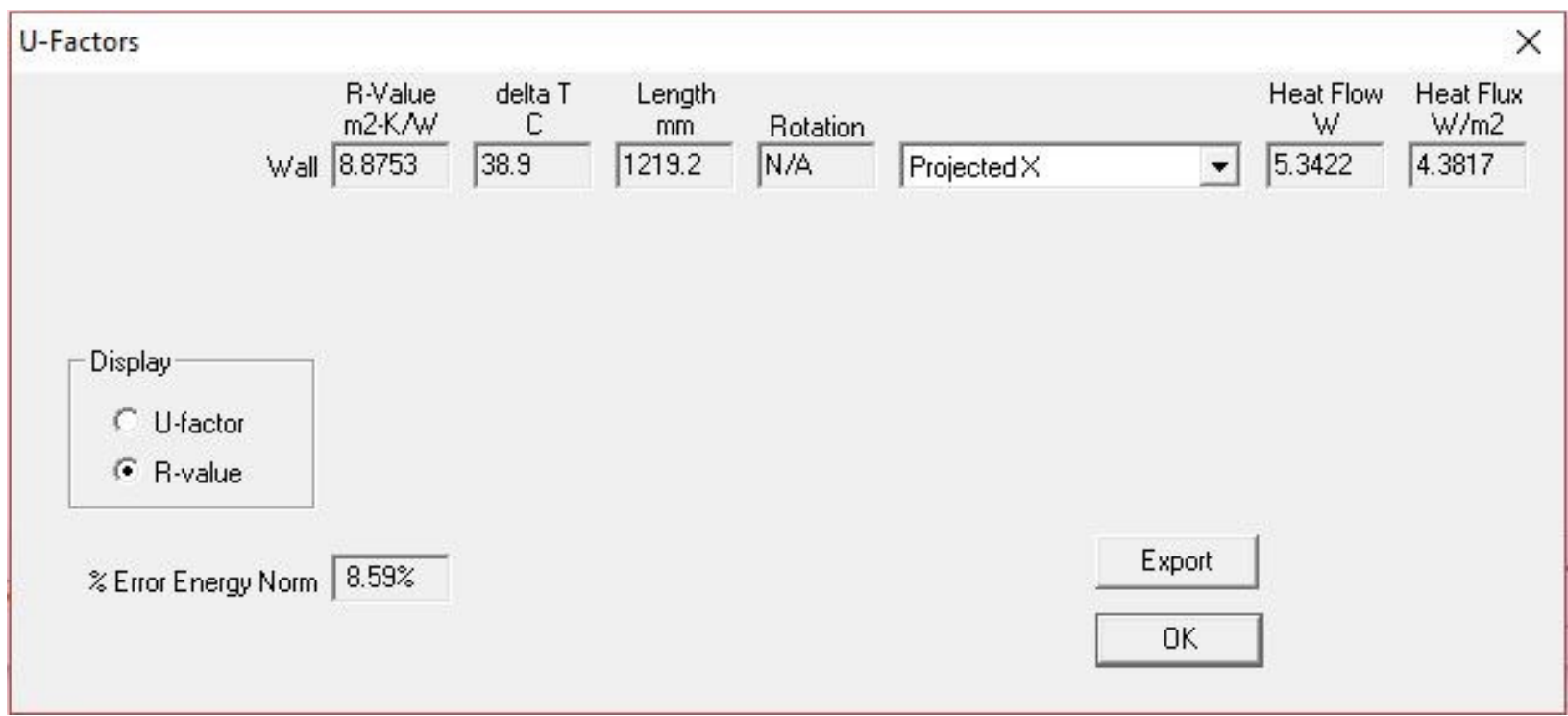

Figure 4-1: U-factor result interface in THERM software

Typically when evaluating wall assemblies, simplifications such as wall symmetry and fictitious stud sizes based on the framing factor are used to create a representative assembly in THERM. These simplifications are used to reduce the number of studs and the overall size required for the geometry. However, it is not common to introduce the significant 3D heat transfer effects through the building envelope. Hence, when a non-homogenous layer (VIP-XPS) is introduced in the building envelope, the common simplifications could be useful.

\subsection{Building Envelope Modelling}

Wood framed envelopes in cold climates are built using various materials at different stud spacing; however, there are common characteristics that exist: the exterior insulation is continuous and there is insulation between the wood stud members. It was mentioned previously that using conventional insulation might not provide sufficient thermal performance to create a thin, high RSI-value building envelope. VIPs in the exterior insulation could provide the necessary thermal performance without significantly increasing the thickness of the envelope. However, cost functions and attempts to protect the VIPs from puncture caused the concept of non-continuous 
exterior insulation with batt insulation inside of the sheathing. This causes multiple two-dimensional sections, which causes problems for building envelope modelling and simulation.

\subsubsection{Multiple Profiles}

Code-built residential building envelopes use structural members, commonly wood lumber, with insulation filled between the cavity and a continuous layer of insulation on the exterior. The envelopes only have a one layer of non-homogeneous properties; the stud-cavity insulation layer. The simulations are performed with the exact geometry, such as the stud widths at the prescribed spacing, or by changing the geometry to incoporate a single, ficitious sized stud. The fictitious stud size is based on the coverage area of wood in the building envelope. The coverage area varies with the wall size, stud thickness and stud spacing. This simplification is common practice when the envelope has continuous exterior insulation. The assumption is shown diagramatically in Figure 4-2 and Figure 4-3.

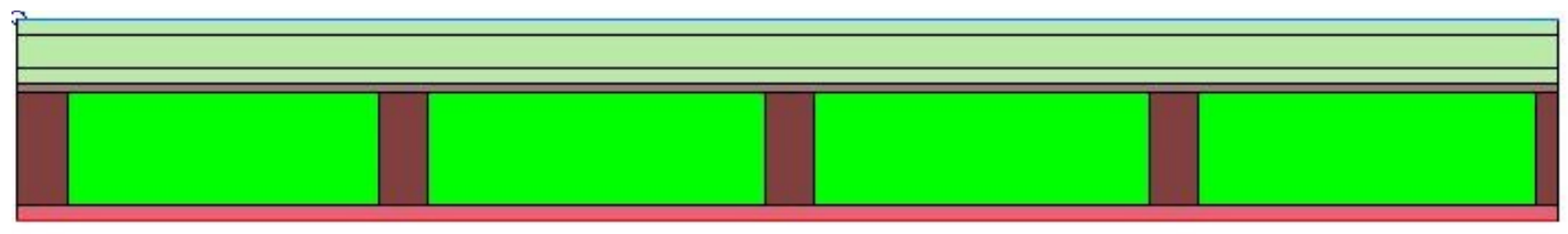

Figure 4-2: Real geometry of a code-built building envelope in THERM

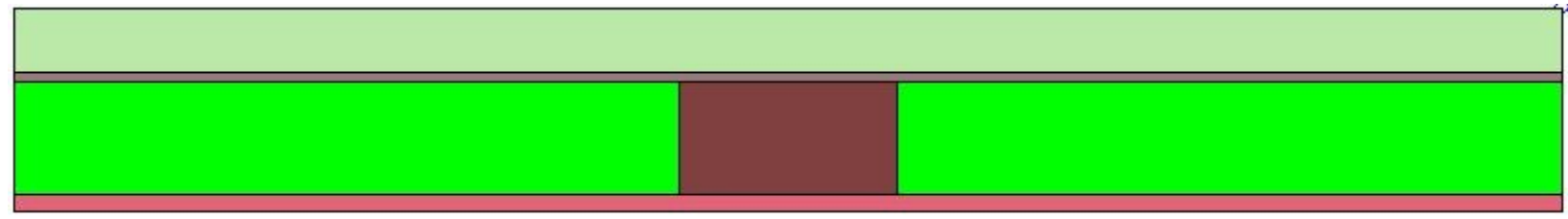

Figure 4-3: Simplified geometry of a code-built building envelope using a fictitious stud size in THERM

However, there are cases when the exterior insulation may not be continuous and further complicates the simulation geometry. The discontinuities in the exterior insulation are can be penetrations in the envelope, changes in insulation materials or materials with non-homogeneousl properties. The simulation's geometry in these cases have an added degree of complexity. Unlike 
code-built envelopes, the wall has more than two cross sections with unique RSI-value. For example, the code-built envelope has two cross sections: the stud and the cavity insulation, both aligned with the continuous exterior insulation. However, if the exterior insulation is split into an insulation and VIP, the new cross sections might be stud-insulation, cavity-insulation, stud-VIP and cavity-VIP. The envelope with two more unique cross sections increases the complexity of the geometry.

To utilize 2D heat transfer software to model non-homogenous wall assemblies, such as those incorporating VIPs, a weighted averaging method has been used in the past. This method involves creating a THERM model for each unique two-dimensional cross section and the height ratio of wall coverage. Using this method, if a VIP is separated into two distinct RSI-values, a large number of models are needed and could become complex. The number of cross sections becomes very significant if the edges of VIPs are considered, instead of using an effective RSI-value.

Since the complexity grows as the effects of VIPs are considered, it may become a deterrent for building designers. A better modelling practice may be required if the number of cross sections required to simulate VIPs in building envelopes hinders their use. To achieve thin, high RSI-value residential building envelopes, designs like VIP composite panels or varying coverage areas of VIPs need to be explored. These designs should not be removed from consideration because they are difficult to simulate because they offer potentially practical ways to attain the thermal performance goals.

\subsubsection{Single Profile}

Modelling complex building envelopes using a single representative cross section would simplify the process. The wall assemblies contain changes in thermal resistance or thermal bridges 
along the cross section. At the interior of the sheathing, the stud framing and cavity insulation are located. At the exterior, it is common to have a homogenous layer of rigid foam board across the entire enclosure. However, when VIPs are installed within the rigid foam, another non-homogenous layer is created. This causes problems when attempting to model as it requires multiple cross sections to be simulated to determine the effective RSI-value. The motivation was to develop a single profile simulation that uses a coverage area ratio for each cross section in the building enclosure.

While performing the simulations and creating the multiple required cross sections to evaluate the wall assembly, it was desirable to develop a less geometrically intensive method. The simulation uses a ratio of exterior insulation to fill the area between the studs. An example schematic of the simulation's geometry is included in Figure 4-4. This new method encompasses the maximum, and minimum heat flows through the assembly and provides an thermal resistance as accurate as the multiple profile simulations. An added benefit is being able to compare the non-homogenous walls easily and evaluating different composite panels designs. The simulation geometry utilizes the area weighted ratios of each unique cross section. For example, VIPs encased in XPS as the exterior insulation on wood-studs contains four unique cross sections exist. The simulation uses varied length of cross section with adiabatic boundary conditions at the edges, interior at the gypsum board and exterior conditions at the exterior of the XPS. The simulation contains two different fictitious stud sizes related to the amount coverage area aligned with the two types of exterior insulation. The sizes of the fictitious studs are calculated with the coverage area of that cross section. 


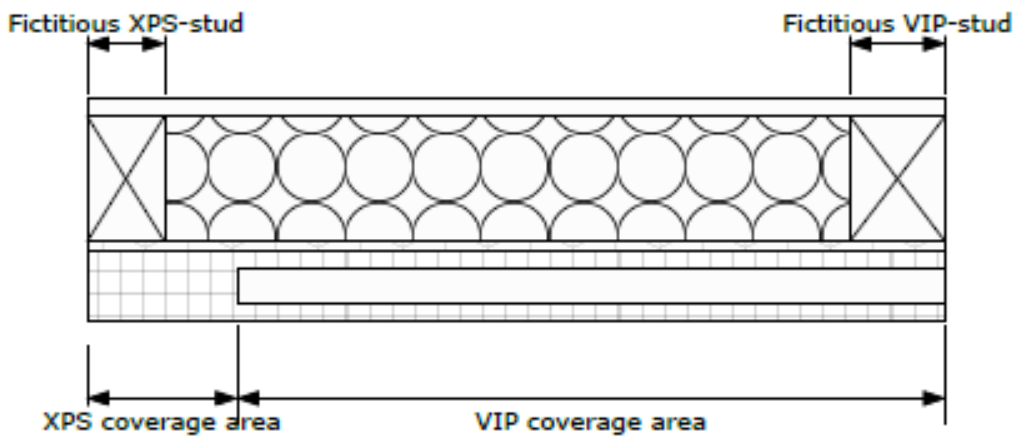

Figure 4-4: Layout of single profile simulation that includes two fictitious stud sizes and coverage areas

Modelling the complex building envelope using a single profile would significantly simplify the process. It may reduce to the necessary cross sections to 1 , which can create an avenue for the integration of VIPs into building envelopes. This would significantly aid in determining the RSI-value of building envelopes and improve the ability to achieve a thin, high RSI-value wall.

\subsection{Iterative Edge Modelling of VIPs}

From the infrared imaging and the experimental data, it was apparent that a reduction in thermal performance existed as the edge of the VIPs. It is commonplace to evaluate building envelopes with VIPs by utilizing the center of panel RSI-value. However, the reduction may be taken into consideration by utilizing an effective RSI-value that is a weighted average area of the edge and center thermal resistances or through data given from the manufacturer. Another solution may be to separate the VIP into components during simulation of calculation. A VIP separated into three components, such as edge, transition, and center of panel where each has a different RSI-value based on experimental data. The modelling geometries quickly become complex but better represent a scenario that would be seen in practice.

Using the THERM software, the VIPs were separated into two and three sections to compare the sensitivity of the values compared to using an effective RSI-value that encompassed the edge effects. The RSI-values used for the edge and transition components were determined 
using experimental data in the guarded hot-box. During the single layer VIP testing, the width of the edge was found through embedding thermocouples along the interior surface. The width of the edge and transition component was observed when the temperature gradient stabilized, such that it maintained steady temperature. The edge RSI-values was measured with the use of embedded heat flux plates and measured temperature difference at the physical edge of the VIP. The RSI-value of the transition component was determined as the midpoint value between the edge and center of panel values. The comparison of effective RSI-values computed from using multiple RSI-values from the iterative method and utilizing an effective RSI-value provided insight into whether separating the VIP into various components should be performed during modelling exercises.

\subsection{Composite Insulation Panels}

The composite insulation panels with VIPs that were tested in the guarded hot-box at steady-state were also modelled using THERM. The panels as designed contained multiple non-homogeneous layers (e.g., wood stud frame and XPS-VIP panel) that caused difficulties in evaluating the effective RSI-value. By using the composite panel, a non-continuous material, as the exterior insulation of the envelope, the envelope was modelled by taking each unique $2 \mathrm{D}$ cross section and averaging based on their coverage area, or height ratio. Height ratio is the height that the $2 \mathrm{D}$ cross section covers divided by the total height of the wall. The height ratio was used for averaging the multiple models to determine an effective RSI-value for the wall assembly. Effective RSI-value of each cross section and the assembly was the output from the models and were compared to the steady-state testing results from the guarded hot-box.

Each assembly was modelled using a symmetrical simplification, such that only half of the wall geometry needed to be included into THERM. The walls were non-homogenous in the vertical 
and horizontal directions, two models needed to be used for each wall design; one that included VIPs and another with XPS. As mentioned prior, the THERM material library contained many of the necessary materials to replicate the main wall construction, however the VIPs did not exist and the thermal resistance per unit thickness was taken from the manufacturer's data sheet. A summary of the materials used are list in Table 4-1. There are two VIP thermal conductivities listed for the different the two manufactures used during the study.

Table 4-1: Material properties used for all the components in the modelling study

\begin{tabular}{l|c}
\hline \multicolumn{1}{c|}{ Material Component } & Thermal Conductivity (W/m·K) \\
\hline Extruded Polystyrene (XPS) & 0.026 \\
Gypsum Wall Board & 0.16 \\
Lumber & 0.14 \\
Plywood (medium density) & 0.17 \\
Fiberglass Insulation & 0.036 \\
Vacuum insulation panels (VIPs) & 0.0036 or 0.0043 \\
\hline
\end{tabular}

The boundary conditions on the left and right of the wall assembly, shown in Figure 4-5 are adiabatic, and the exterior and interior surfaces have "exterior winter", denoted by the blue surface, and "interior vertical surface", denoted by the red surface, boundary conditions respectively. They set the temperature of the interior wall at $21.1^{\circ} \mathrm{C}$ and the exterior $-17.8^{\circ} \mathrm{C}$ with film coefficients of $2.44 \mathrm{~W} / \mathrm{m}^{2} \mathrm{~K}$ and $26 \mathrm{~W} / \mathrm{m}^{2} \mathrm{~K}$, respectively. The boundary conditions used were within the THERM library and were used for other wall assembly models within literature [47].

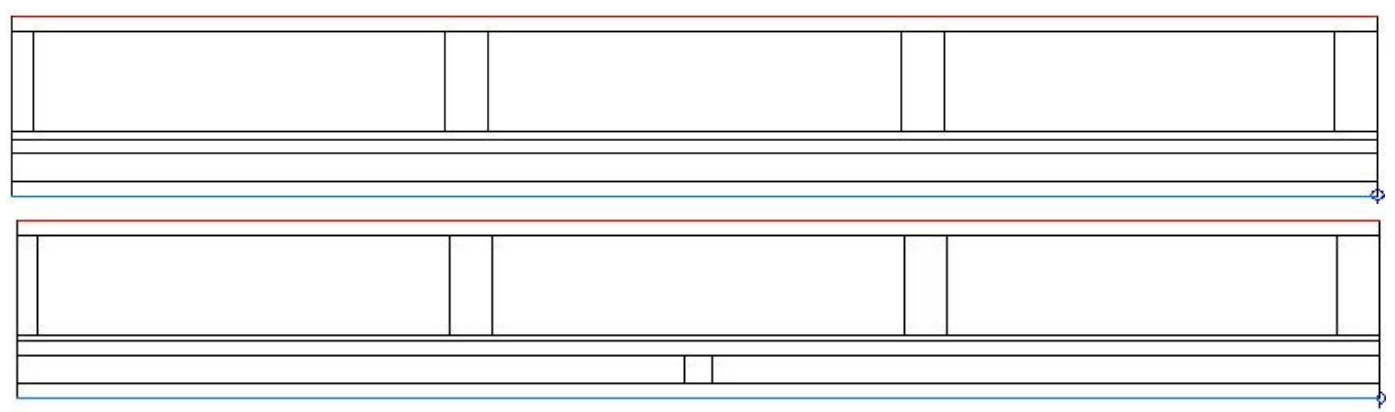

Figure 4-5: THERM cross sections of Test 3 using. Top is only XPS, bottom is VIP with XPS spacers. 
The composite insulation panels were also evaluated using a single profile, as seen in Figure 4-4. The profile used the ratio of coverage areas for the building envelope assembly, including the composite insulation panel, for building the model geometry. The model used the same boundary conditions and material properties from the modelling using multiple cross

sections, however, the dimensions were changed. The effective RSI-value of the assembly was a result of the single profile model and was compared to the steady-state testing and multiple cross sections with a weighted average. This analysis provided an accurate assessment of the thermal performance of the composite insulation panels as well as the viability of the modelling complex building envelopes using a single cross section.

\subsection{Summary}

This chapter provided some background information on THERM and its inputs, the necessary steps to model building envelopes, the modelling approach for the composite insulation panels and the desired outputs. The single and multiple profile methods for modelling were explained and a discussion on how they performed in THERM was presented. Analyzing the edge of a VIP as multiple separate sections was examined and most effective approach to evaluate the VIP through simulation was discussed. Finally, the material properties and boundary conditions for the composite insulation panels for the modelling study were presented. The next chapter will present the experimental and modelling results in detail. 


\section{Chapter: Results}

The use of VIPs in building envelopes for creating a high RSI-value building envelope was evaluated using experimental and computer simulation with promising results. Testing a single layer of VIPs at steady-state conditions in the guarded hot-box indicated a change in thermal resistance from the center of the panel to the edge as well as a measured effective RSI-value of the entire layer. After adding the second layer of VIPs in three different orientations, the changes in heat flux and effective RSI-values were observed and compared. Finally, the effectiveness of adding VIPs between typical insulation materials for protection purposes was evaluated. The effective thermal resistance and maximum and minimum heat fluxes, were measured as well as the temperature variations within the composite panels.

The modelling approach used in this study involved comparing modelling methods and techniques for the evaluation and implementation of VIPs into building envelopes. Due to the non-homogeneous nature of VIPs, complications can arise when applying a thermal resistance value to the panel. The full wall assemblies from the composite panel were modeled in THERM using various techniques. These included the current standard of evaluating an entire symmetrical section with appropriate stud spacing, and a simplified single cross section. Evaluating the methods' accuracies and validities were performed through comparing the effective thermal resistance from THERM to the experimental steady-state results.

\subsection{Single Layer VIP}

The single layer VIP was the initial experimental evaluation performed at steady-state conditions within the guarded hot-box. The objective of the test was to determine the effective RSI-value of a VIP layer as well as the change in heat fluxes between the center and the edge of a 
panel. This information is important because a large discrepancy between those points exists, but the exact value is unknown.

\subsubsection{Effective Thermal Resistance of a Single Layer}

Within the surround panel, a $1.8 \mathrm{~m}\left(72\right.$ ") by $1.6 \mathrm{~m}$ (66") layer composed of $457 \mathrm{~mm}\left(18^{\prime \prime}\right)$ by $558 \mathrm{~mm}$ (22") VIPs are tiled in a 4 by 3 grid. The VIPs were attached to the structural sheathing through the pre-existing adhesive, as shown in Figure 5-1. To evaluate the thermal resistance of a single layer of VIP at steady-state conditions, the power input to the metering chamber and the surface temperatures on both sides of the specimen and metering chamber walls was measured. During the test period, the metering chamber required $40.9 \mathrm{Wh} \pm 0.6 \mathrm{Wh}$ to maintain a temperature difference of $34.0^{\circ} \mathrm{C} \pm 0.6^{\circ} \mathrm{C}$ across the entire wall assembly. Furthermore, the heat flow through the metering chamber walls was found to be negligible based on the temperature measurements, known surface area, and thermal resistance, since the heat flow was less than $1 \%$ of the total heat flow through the specimen. Therefore, an effective RSI-value for the assembly of XPS-VIPPlywood was $3.79 \mathrm{~m}^{2} \mathrm{~K} / \mathrm{W} \pm 0.2 \mathrm{~m}^{2} \mathrm{~K} / \mathrm{W}$ for the $1.83 \mathrm{~m}^{2}$ metered area. Table $5-1$ outlines the steady-state test conditions, where the specimen surface temperatures, heat input to the metering chamber, elapsed time of the test period and the calculated effective thermal resistance are listed. 


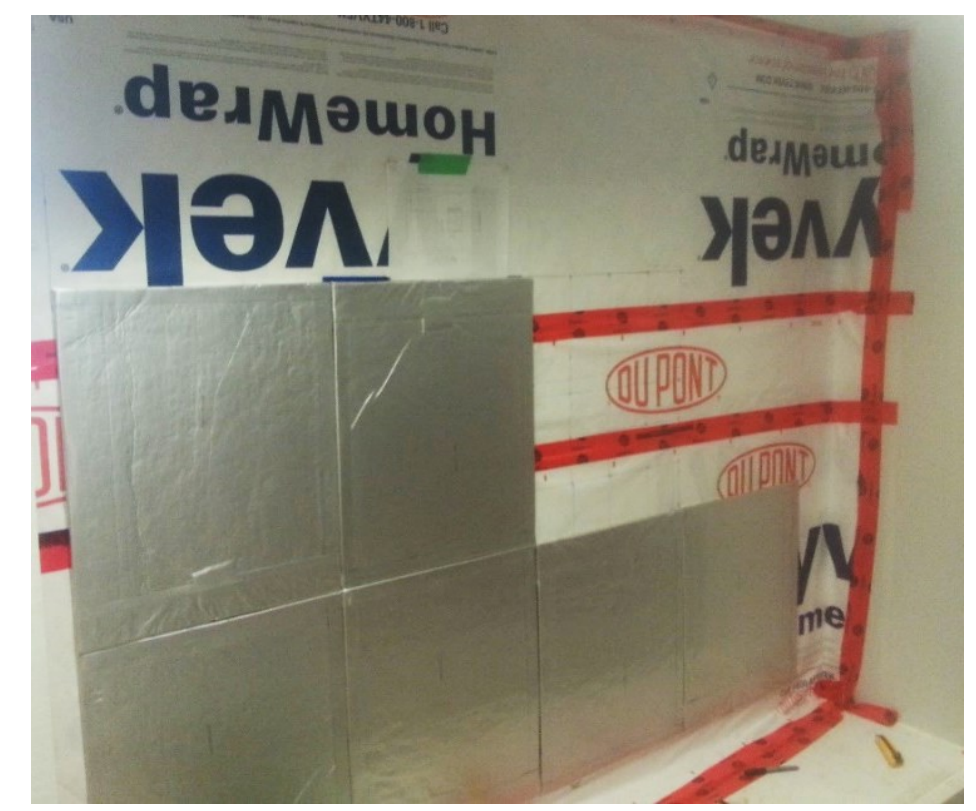

Figure 5-1: Single layer VIP configuration inside guarded hot-box

Table 5-1: Steady-state test conditions for a single VIP layer

\begin{tabular}{l|c}
\hline Test Conditions & \\
\hline Cold Specimen Surface $\left[{ }^{\circ} \mathrm{C}\right]$ & -12.1 \\
Hot Specimen Surface $\left[{ }^{\circ} \mathrm{C}\right]$ & 22.0 \\
Heat Input to the Metering Chamber $[\mathrm{Wh}]$ & 40.8 \\
Elapsed Time $[\mathrm{h}]$ & 2.5 \\
Effective RSI $\left[\mathrm{m}^{2} \mathrm{~K} / \mathrm{W}\right]$ & 3.8 \\
\hline
\end{tabular}

\subsubsection{Thermal Resistance Center, Corner, and Edge of the Panel}

In addition to determining the effective thermal resistance of a VIP layer through the steady-state testing, the changes in thermal resistance along the VIP layer was important to evaluate during the study. It was studied using embedded heat flux plates and thermocouples at steady-state conditions.

Similar to the initial tests of a single VIP layer, the temperature sensors were embedded on both sides of the VIP and heat flux sensors were placed at the XPS-VIP interface. After reaching steady-state conditions according to ASTM C-1363, the embedded temperature sensors were averaged based on their location on the VIP; characterized as a center, edge, or corner. Using 
Equation 2 (p.42), the thermal resistance value of the panels was calculated. Table 5-2 outlines the temperature and heat flux measurements. Results indicate a large change in temperature from the center and corner, while the change between the corner and edge is significantly smaller. The data showed that a significant drop in performance occurs at the edge and corner compared to the center of panel. Table 5-3 shows the change in measured heat flow and the percentage in reduction compared to the center. It was found that the maximum reduction of RSI-value of the VIP was $36 \%, 2.4 \mathrm{~m}^{2} \mathrm{~K} / \mathrm{W}$ at the center to $1.5 \mathrm{~m}^{2} \mathrm{~K} / \mathrm{W}$ at the corner.

Table 5-2: Measured temperature and heat flux around VIP

\begin{tabular}{l|ccc}
\hline & Warm Side $\left[{ }^{\circ} \mathrm{C}\right]$ & Cold Side $\left[{ }^{\circ} \mathrm{C}\right]$ & Heat Flux $\left[\mathrm{W} / \mathrm{m}^{2}\right]$ \\
\hline Center & 21.2 & 5.8 & 6.5 \\
Seam & 17.9 & 5.8 & 7.4 \\
Corner & 17.6 & 5.8 & 7.8
\end{tabular}

Table 5-3: Difference in thermal resistance at points on a VIP

\begin{tabular}{l|lll}
\hline & Center & Edge & Corner \\
\hline RSI Value $\left[\mathrm{m}^{2} \mathrm{~K} / \mathrm{W}\right]$ & 2.4 & 1.6 & 1.5 \\
\% Reduction from Center & - & $32 \%$ & $36 \%$ \\
\hline
\end{tabular}

\subsubsection{Creation of a Temperature Heat Map}

Behind a single VIP located within the metering area, a 5 by 6 grid of thermocouples were embedded and the temperature gradient was measured. Each thermocouple was spaced $112 \mathrm{~mm}$ (4.4") horizontally and $114 \mathrm{~mm}(4.5 ")$ vertically and the temperatures between the points were interpolated. Due to the small distances and density of sensors, it was determined that a linear interpolation was a sufficient means to construct a heat map using the Microsoft Excel software. In Figure 5-2 and Figure 5-3, the thermocouple layouts for a single VIP before and after installation of the instrumentation are shown. 


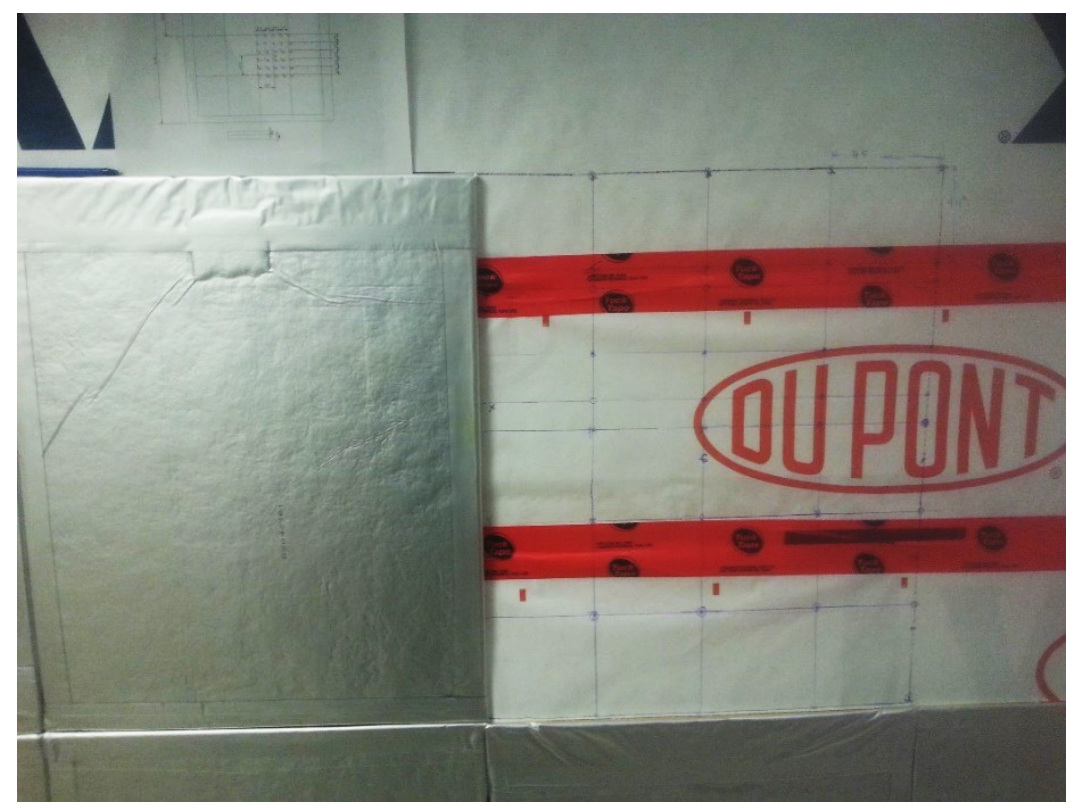

Figure 5-2: Thermocouple layout behind a single VIP without thermocouples

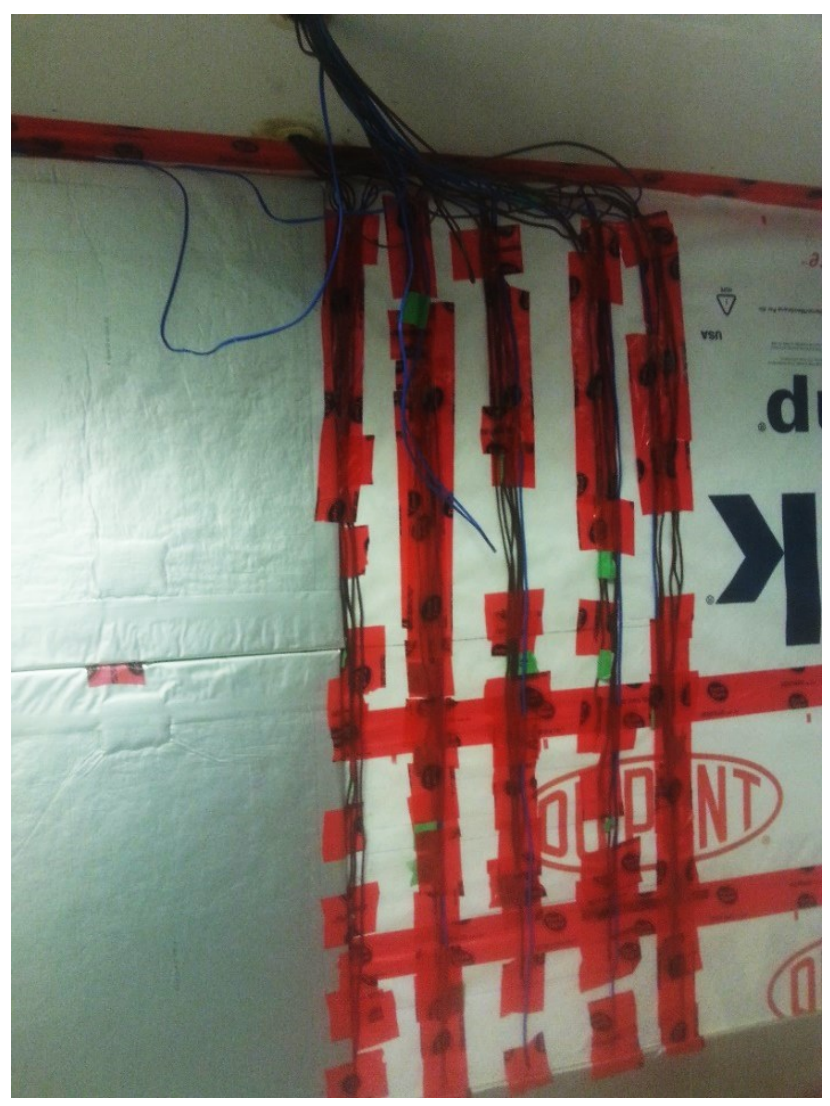

Figure 5-3: Thermocouple layout behind a single VIP with thermocouples 
The heat map was created as a means to easily visualize the temperature gradients within the building envelope. Each cell of spreadsheet contains a temperature and is coloured based on the scale and become elements of the heat map. Each cell contained a temperature input obtained from a measured value from the embedded thermocouple or the cell contained a temperature that has been linearly interpolated from two inputs. In this fashion, the temperatures between two adjacent columns linearly increased and sufficient granularity was achieved to effectively view the trends of the temperature gradients. After a temperature is calculated or assigned to a cell, a colour based on a blue to red scale fills the cell. The colour scale was based on the maximum and minimum temperature, where the brightest red and blue represent the maximum and minimum temperatures, respectively. The outer boundaries of the heat map were aligned with the perimeter of the VIP, such that the edges of the heat map in Figure 5-4, were temperatures at the edges of the VIP.

In Figure 5-4, the temperature inputs to spreadsheet were associated to each thermocouple in the instrumentation diagram in Figure 3-12. It is important to note the variation in temperature along the edges. The measured temperatures varied between $14.8^{\circ} \mathrm{C}$ to $20.6^{\circ} \mathrm{C}$ along the perimeter of the VIP, while the temperature varied significantly less (between $20.6^{\circ} \mathrm{C}$ to $21.3^{\circ} \mathrm{C}$ ) at $102 \mathrm{~mm}$ from the perimeter to the center. The measurement discrepancies between the edges and center showed that the VIP did not perform homogeneously and each edge performed differently than the adjacent VIP edge. The heat map qualitatively showed the interface temperature behind the VIP and confirmed that the edges are a point of lower thermal resistance. The heat map could be used instead of infrared thermal imaging when necessary but multiple measurement points along the edges and center are required to contain sufficient granularity. The study showed that without a 
measured temperature at each edge, the heat map would not encompass the correct temperature variations.

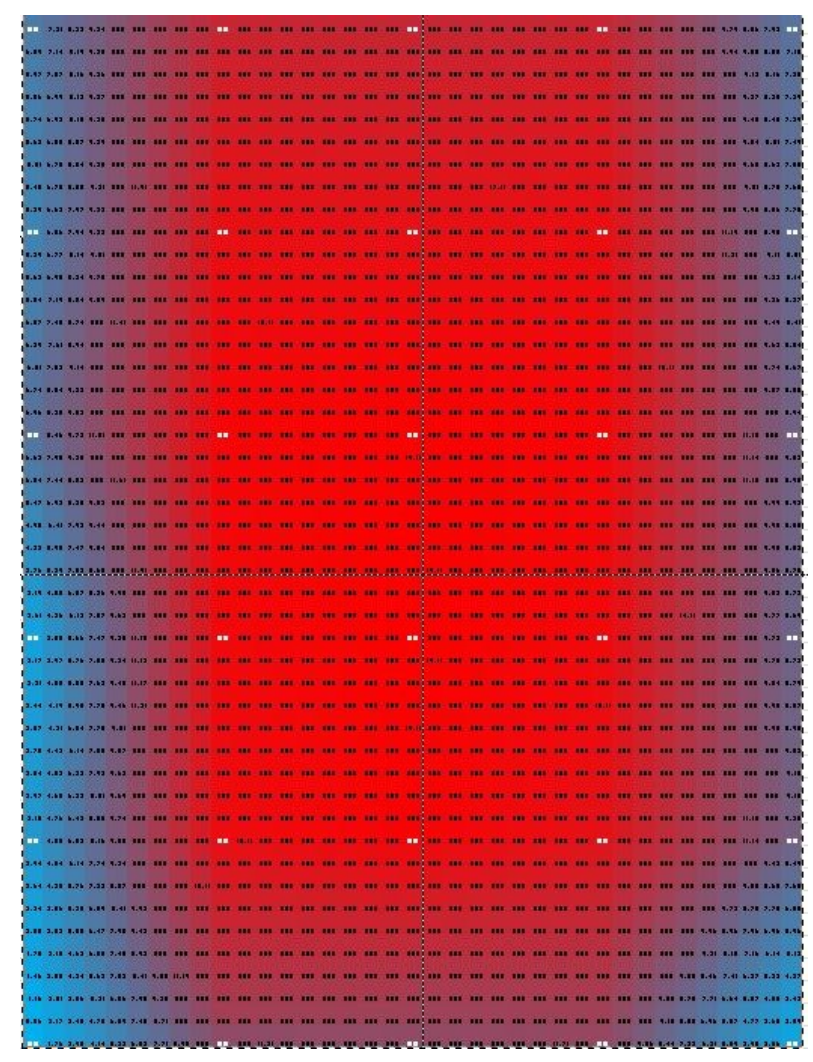

Figure 5-4: VIP heat map using Microsoft Excel with measured temperatures are indicated by the white numbers

\subsection{Double Layer VIP}

A second layer of VIPs was added in order to study the effect that offsetting would have on temperature and heat flux variation within the building envelope. Three different offsets of the second VIP layer were used and they represent the extremes of potential designs. The offset of the second layer of VIPs is labeled by where the center of a panel on the second layer aligns with a panel on the first layer (e.g., center-center, center-seam, and center-corner). These assemblies were evaluated at steady-state conditions within the guarded hot-box facility and the test period required multiple days. This test period included the time to reach steady-state conditions and a data 
collection period after reaching steady-state. It was also necessary to ensure after each test that all VIPs were intact and that no punctures or failed panels existed in the assembly through thermal imaging.

\subsubsection{Thermal Resistance of Each Offset}

It was anticipated that offsetting the second layer of VIPs would strongly affect the temperature variations of the assembly; however, it was unknown whether it could improve the effective thermal resistance of the assembly. By using the same VIPs, a number of thermal bridges remain constant, but the maximum and minimum warm surface temperature difference for different offets would be measured. These values were measured to provide an indicator of homogenous thermal resistance as the temperature difference becomes smaller.

The steady-state temperatures at each interface of the assembly are shown in Figure 5-5 to Figure 5-7. In the graphs "Climate" is the surface temperature in the climate chamber, "Metering" represents the surface temperature in the metering chamber and XPS-XPS, XPS-VIP, VIP-VIP, and VIP-PLY are the interfaces (cold-warm) within the wall assembly. The graphs illustrate the temperature at each interface over the test and measurement period and clearly show that minimal temperature variation exists. This is a strong indicator that the apparatus has met steady-state conditions. 


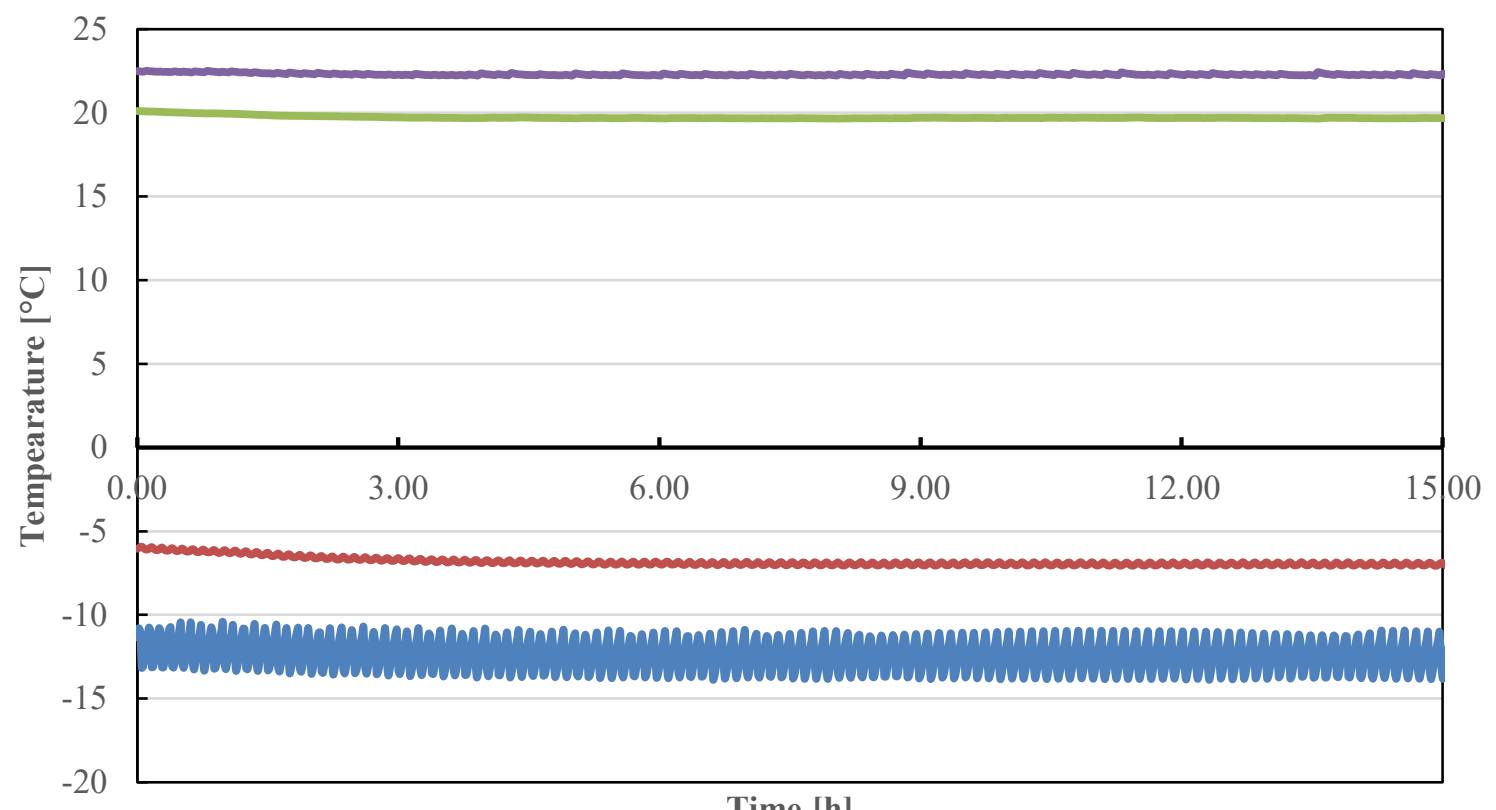

Time $[\mathrm{h}]$

Climate $\longrightarrow$ XPS XPS $\longrightarrow$ VIP PLY $\longrightarrow$ Metering

Figure 5-5: Steady-state temperature profile at each material interface of center-center configuration

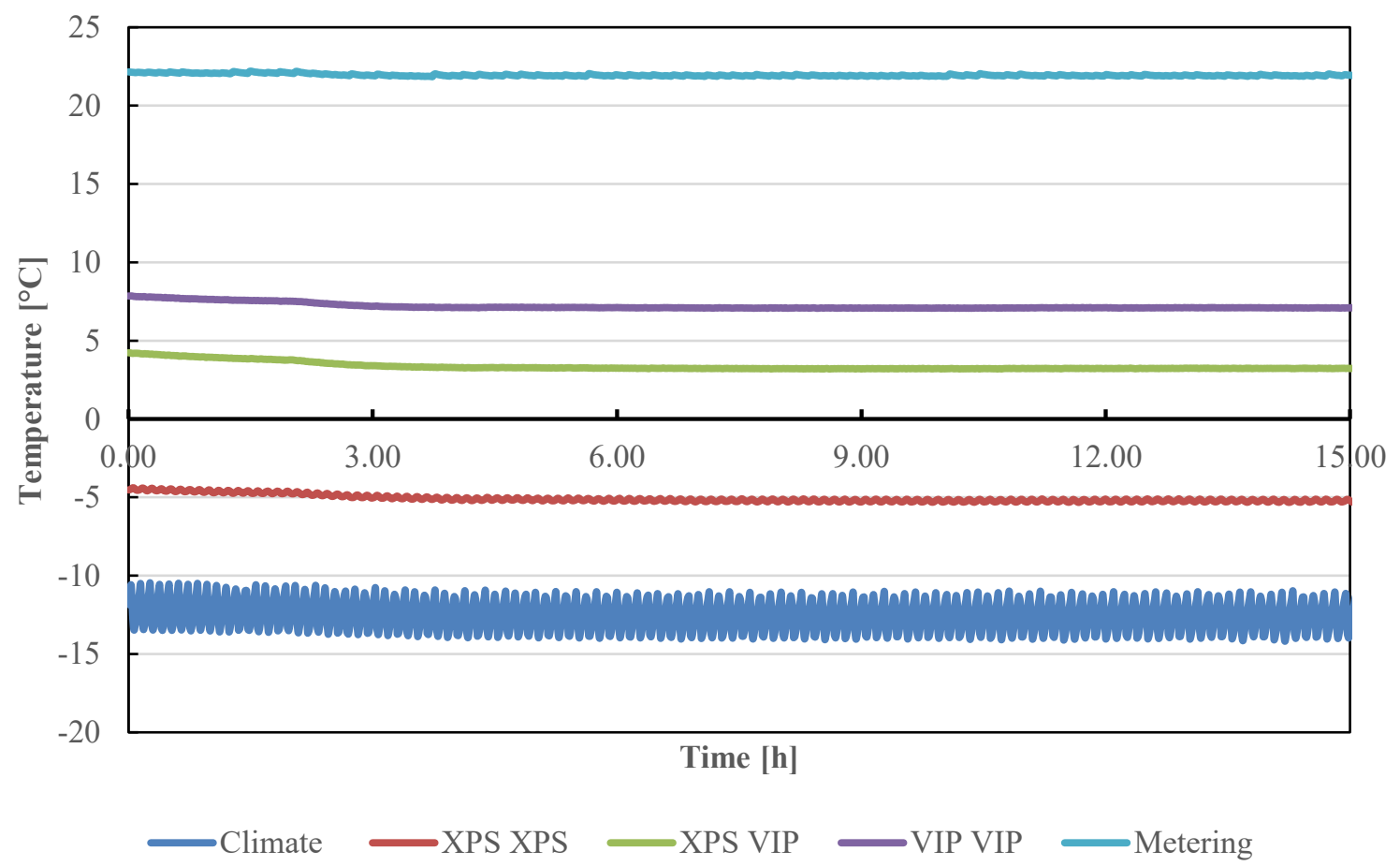

Figure 5-6: Steady-state temperature profile at each interface of center-seam configuration 


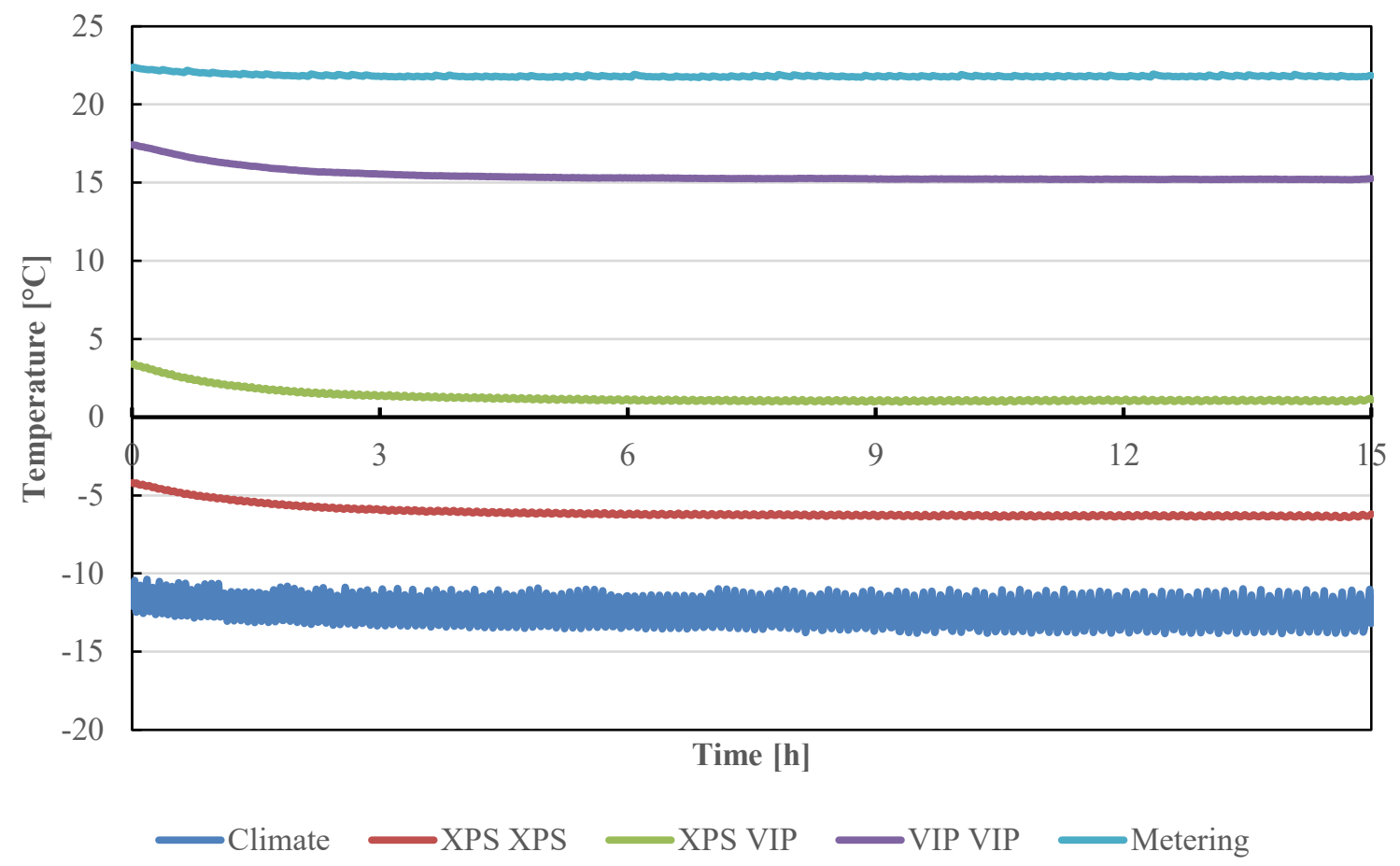

Figure 5-7: Steady-state temperature profile at each material interface of center-corner configuration

The test conditions and measured values for each orientation are listed in Table 5-4, along with the power input from the heaters in the metering chamber and the calculated effective thermal resistance of each assembly. The effective thermal resistance was calculated by using Equation 1 (p.41). The effective RSI-values calculated contained the RSI-values of the plywood, two layers of VIP and the $50 \mathrm{~mm}$ of XPS on the exterior. The study highlighted that changing the second VIP layer orientation marginally affected the effective RSI-value $\left(0.5 \mathrm{~m}^{2} \mathrm{~K} / \mathrm{W}\right)$, but could still affect the variations in heat flux and temperature distribution through assembly

Table 5-4: Test conditions for each double layer VIP configuration

\begin{tabular}{l|ccc}
\hline Conditions & Center-Center & Center-Edge & Center-Corner \\
\hline Cold Specimen Surface $\left[{ }^{\circ} \mathrm{C}\right]$ & -11.2 & -12.8 & -11.9 \\
Hot Specimen Surface $\left[{ }^{\circ} \mathrm{C}\right]$ & 22.3 & 22.0 & 22.2 \\
Heat Input to the Metering Chamber $[\mathrm{Wh}]$ & 11.7 & 13.2 & 13.4 \\
Elapsed Time $[\mathrm{h}]$ & 1.0 & 1.0 & 1.0 \\
Effective RSI $\left[\mathrm{m}^{2} \mathrm{~K} / \mathrm{W}\right]$ & 5.3 & 4.8 & 4.7 \\
\hline
\end{tabular}




\subsubsection{Variation of Heat Flux through Double Layered VIPs}

It was desirable to find the changes in heat flux through the assemblies. The heat flux sensors remained in the same location for each test: center, corner, and seam at the interface between the two layers of VIPs. At these fixed positions, the heat flux plates experience and measured the heat flux variations expected within the assembly. While the sensors changed the heat flow through the assembly, the low thermal resistance and guarded metering area did not compare to the VIPs. Since the assembly was highly insulated, embedding the heat flux plates did not substantially alter the readings or heat flow. The heat flux measurements were taken during the same tests as described in Section 5.2.1, as such the results were also at steady-state conditions.

The heat flux variation in the assemblies, shown in Figure 5-8, was plotted based on the percent difference between the maximum and minimum heat flux through the assembly. The results proved that the center-corner orientation significantly limits the spread in heat flux through the assembly, while the center-center and center-edge yield a higher difference, most likely due to the low thermal resistance points of the VIP of both layers remaining aligned. The large variations in heat flux are due to the instrument's sensitivity to temperature and convective current variations caused by the cycling of the refrigeration unit. Generally, the variations are limited when the sensors are embedded further into the wall assembly toward the interior. As such, when evaluating full wall assemblies, the temperature disruptions at interfaces closer to the interior are moderated and the sensor contains less oscillation. 


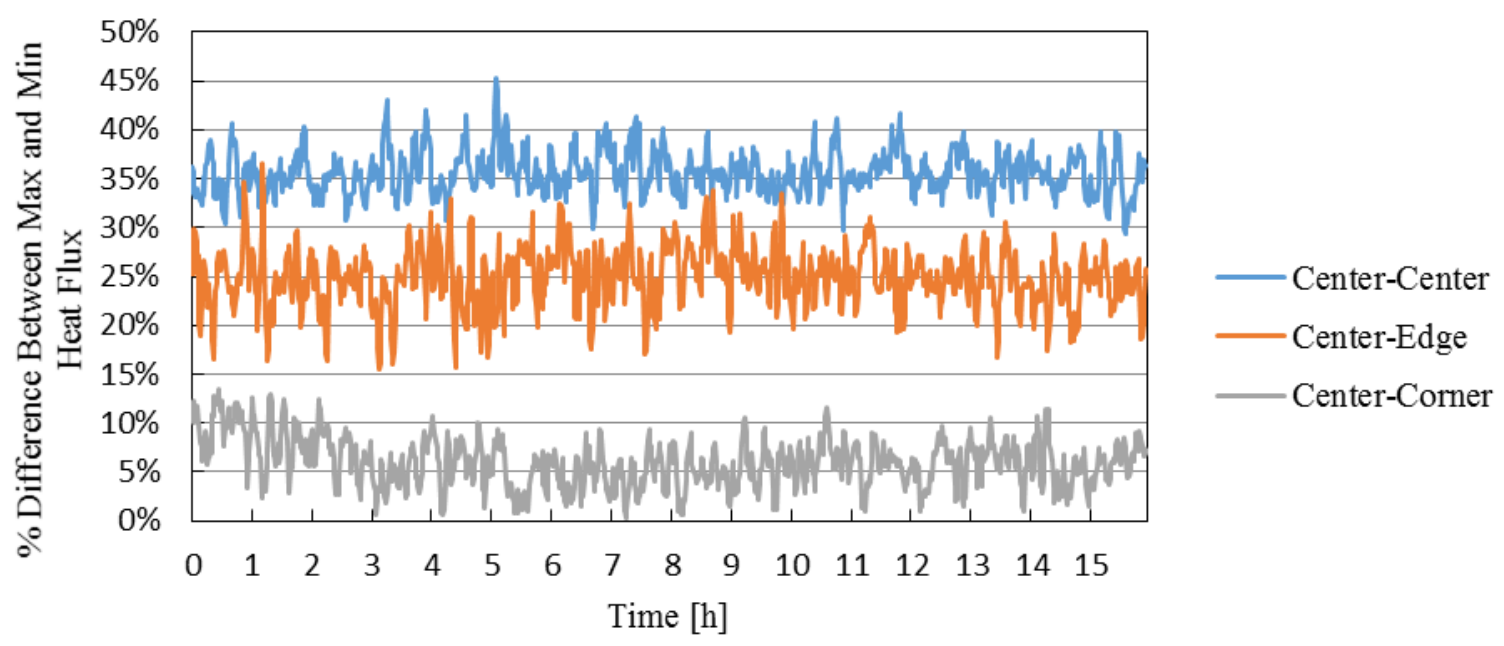

Figure 5-8: Difference between maximum and minimum heat flux over the test period

The measured values are supported by the thermal images taken after the metering and guarded chamber were removed from the guarded hot-box. The infrared photos taken of the double layers using center-center offset, and center-seam and center-corner offsets, shown in Figure 5-9 to Figure 5-11, show the large temperature variations present at the interior surface. In Figure 5-9, the perimeter of the VIPs is easily seen by the green and blue lines in the center of the figure. However, in Figure 5-11, when the VIPs were configured to center-corner orientation, the temperature difference on the surface is small, especially in comparison to Figure 5-9. In addition, the images show how offsetting these panels moderate the variations at the VIP-PLY interface. The measured values and IRT showed the same trends that indicate the potential that offsetting VIPs could have on limiting temperature variations within the wall envelope. 


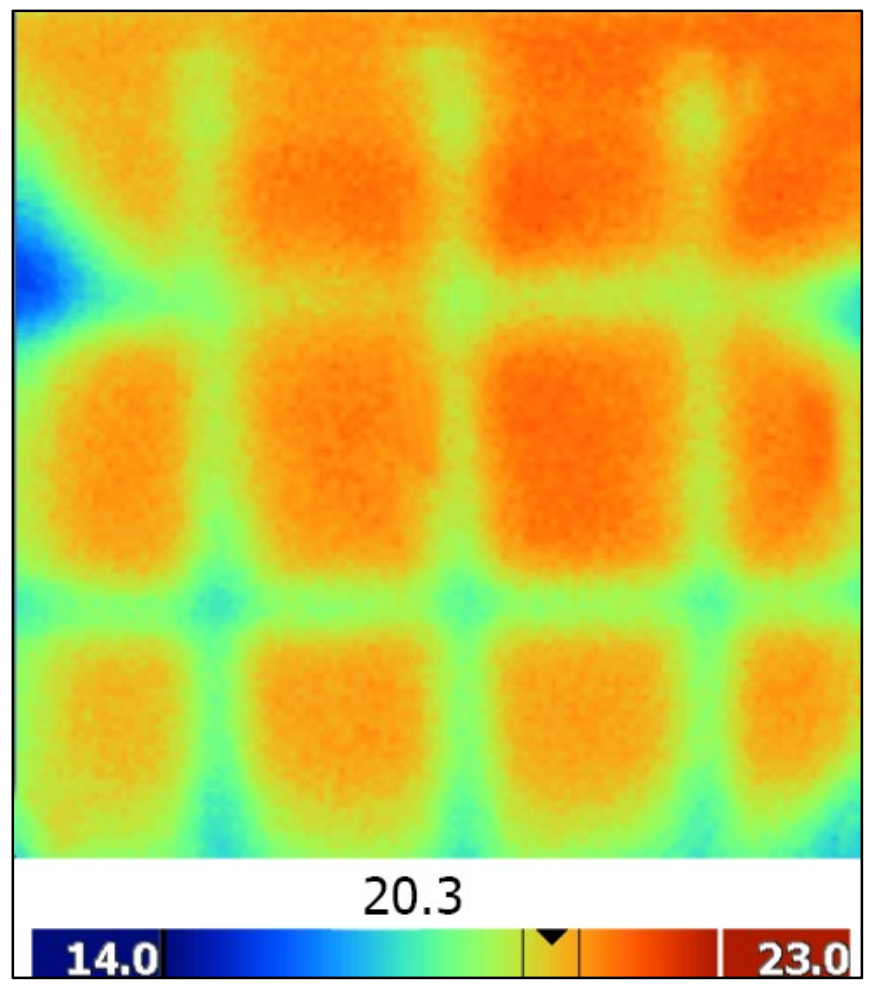

Figure 5-9: Infrared photo of double VIP layer center-center

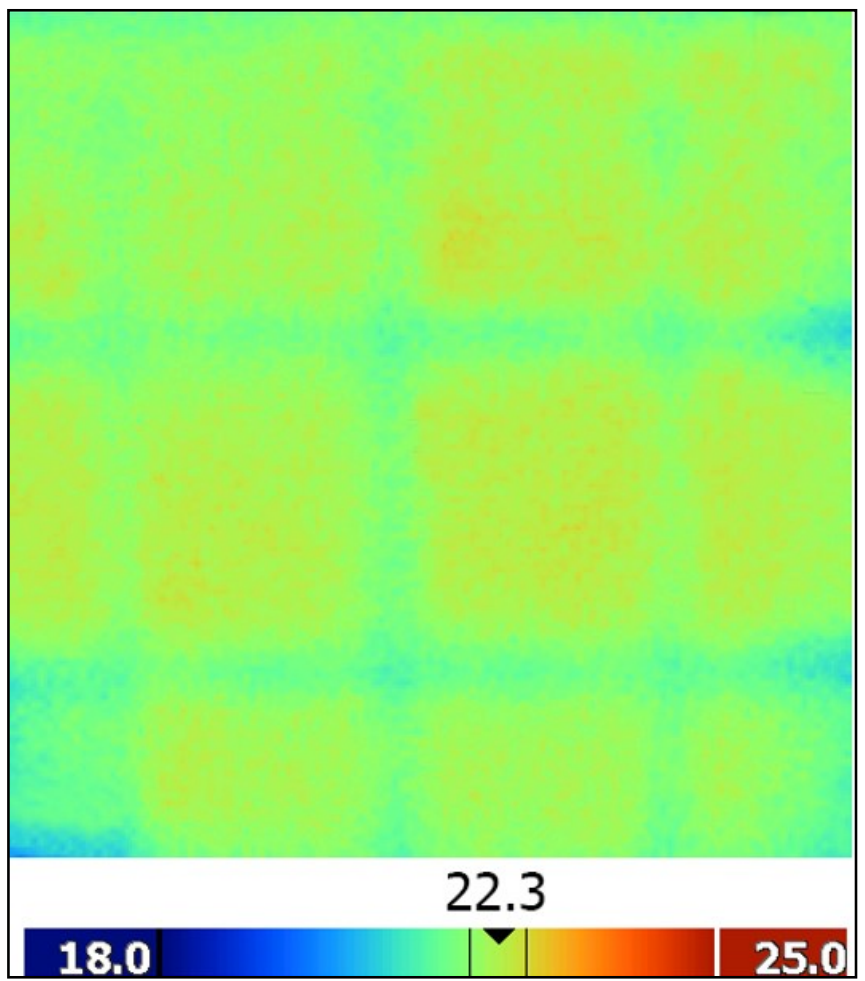

Figure 5-10: Infrared photo of double VIP layer center-seam offset 


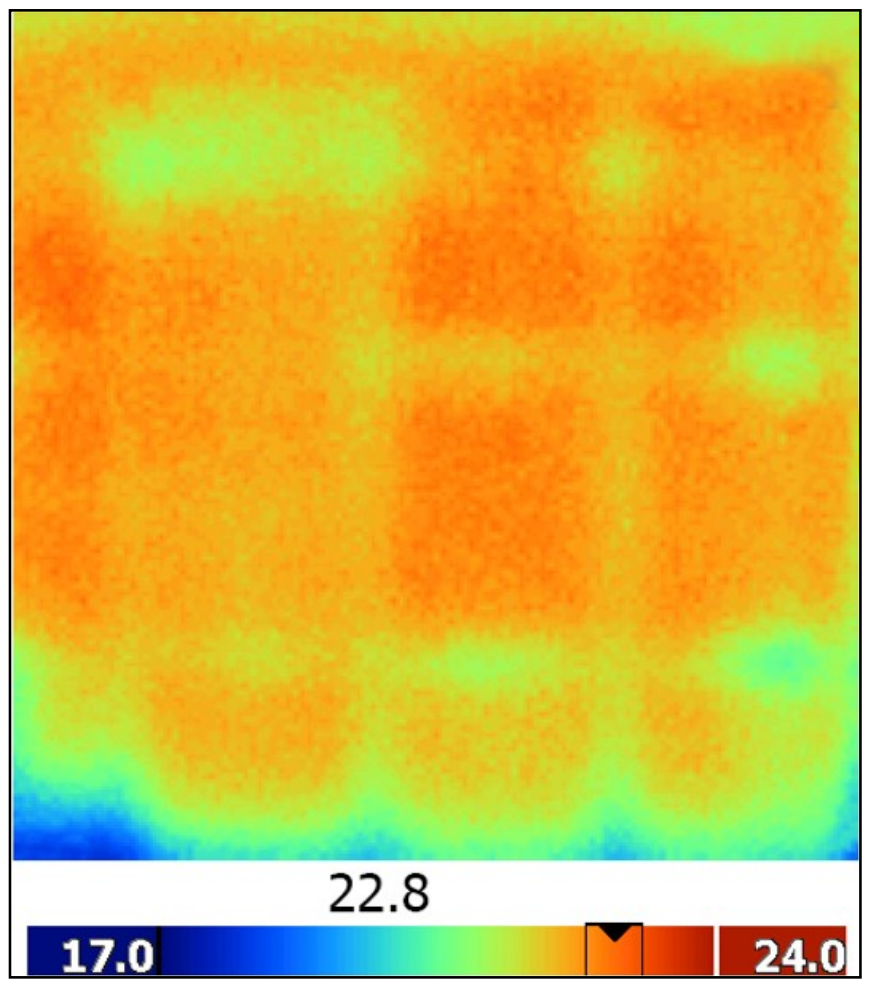

Figure 5-11: Infrared photo of double VIP layer center-corner offsets

\subsection{Entire Wall Assembly}

After the effective thermal resistance of the VIP edge was determined, the effects of VIPs installed into a full wall assembly were studied. The application with the most potential of integration into building enclosures involves encasing the VIPs in insulation with spacing for mechanical fasteners. The motivation behind encasing the VIPs within the composite panels is to minimize the risk of puncture. The thermal performance reduces drastically if the VIPs become punctured therefore surrounding the VIP with a lower, albeit standard, thermally resistant insulation material protects the VIP and maintains above standard of effective thermal performance. 
The composite panels were tested using the guarded hot-box under steady-state conditions and modelled using the THERM software. During the steady-state testing, the composite panels were built as scaled representations of how they would be installed in a building envelope. The VIP coverage, XPS coverage, and framing factor were mimicked to properly evaluate the scaled composite panels. During the modelling studies, the full wall dimensions were used as oppose to the scaled dimensions within the guarded hot-box as the simulation geometry. The stud spacing, VIP coverage, and XPS coverage were all varied and five different main wall assembly designs were created and evaluated using steady-state testing and THERM simulations.

\subsubsection{Effective RSI-value}

The steady-state evaluation of the wall assemblies was performed over a longer period compared to the period to evaluate the single layer of VIPs. This is due to the additional amount

of time required for the heat to permeate through the wall and reach steady-state conditions and to the full wall assembly having a greater thermal mass as well as a large effective thermal resistance value. As such, a measurement period of 20 hours was used to determine an accurate effective RSI-value since the steady-state checks require the heat input to vary less than $5 \%$. The walls remained under test conditions for approximately 4 days before the measurement period was conducted. The results from the experiments are summarized into Table 5-5, which includes the interior and exterior surface temperatures, energy input to the metering chamber and the calculated effective RSI-value of the wall assembly design. 
Table 5-5: Summary of steady-state test results over 20 hour period

\begin{tabular}{l|cccc}
\hline Test & Climate $\left({ }^{\circ} \mathrm{C}\right)$ & Metering $\left({ }^{\circ} \mathrm{C}\right)$ & Heat Input $(\mathrm{Wh})$ & Effective RSI $\left(\mathrm{m}^{2} \mathrm{~K} / \mathrm{W}\right)$ \\
1 & $-13.3 \pm 1.4$ & $23.0 \pm 0.6$ & $139.9 \pm 0.5$ & $8.97 \pm 0.27$ \\
2 & $-10.0 \pm 1.1$ & $23.2 \pm 0.5$ & $127.1 \pm 0.5$ & $8.97 \pm 0.28$ \\
3 & $-13.7 \pm 1.4$ & $23.1 \pm 0.5$ & $159.4 \pm 0.5$ & $8.44 \pm 0.23$ \\
4 & $-11.8 \pm 1.4$ & $23.4 \pm 0.5$ & $126.0 \pm 0.5$ & $9.07 \pm 0.30$ \\
5 & $-11.8 \pm 1.0$ & $23.3 \pm 0.5$ & $126.0 \pm 0.5$ & $8.97 \pm 0.27$ \\
\hline
\end{tabular}

\subsubsection{Temperature Profile at Four Cross Sections}

Thermocouples were embedded at each interface in the wall assembly and a temperature profile was determined through the main wall. There were four thermocouples embedded within each interface, one for each unique cross section the wall contains. The temperatures are shown for the 20-hour measurement period for all four cross sections of Test 1 and Test 2 is shown in Figure 5-12 to Figure 5-15 and Figure 5-16 to Figure 5-19, respectively. The temperature sensor on one side of the VIP in each test (cold side of VIP in Test 1, warm side of VIP in Test 2) was not added to the composite panel as an oversight during their construction. These graphs show that there is a limited temperature variation through the measurement period and the temperature difference between interfaces indicate which materials have a greater thermal resistance or act as better insulators. 


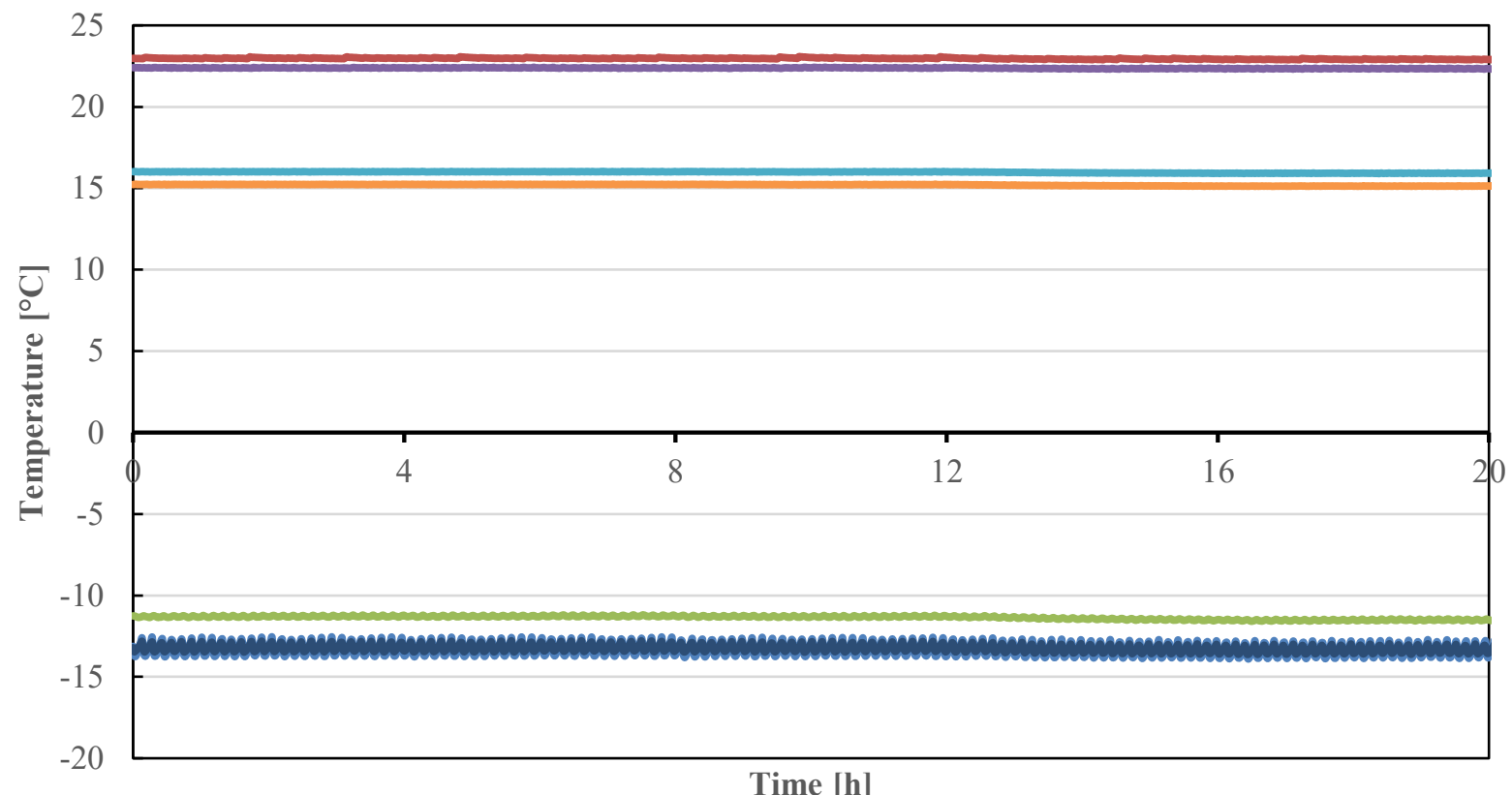

Time $[\mathrm{h}]$

$\longrightarrow$ Climate $\longrightarrow$ SIDE XPS $\longrightarrow$ XPS VIP $\longrightarrow$ XPS PLY $\longrightarrow$ PLY FIB $\longrightarrow$ FIB DRY $\longrightarrow$ Metering

Figure 5-12: Steady-state temperature profile at the VIP-stud cross section of Test 1

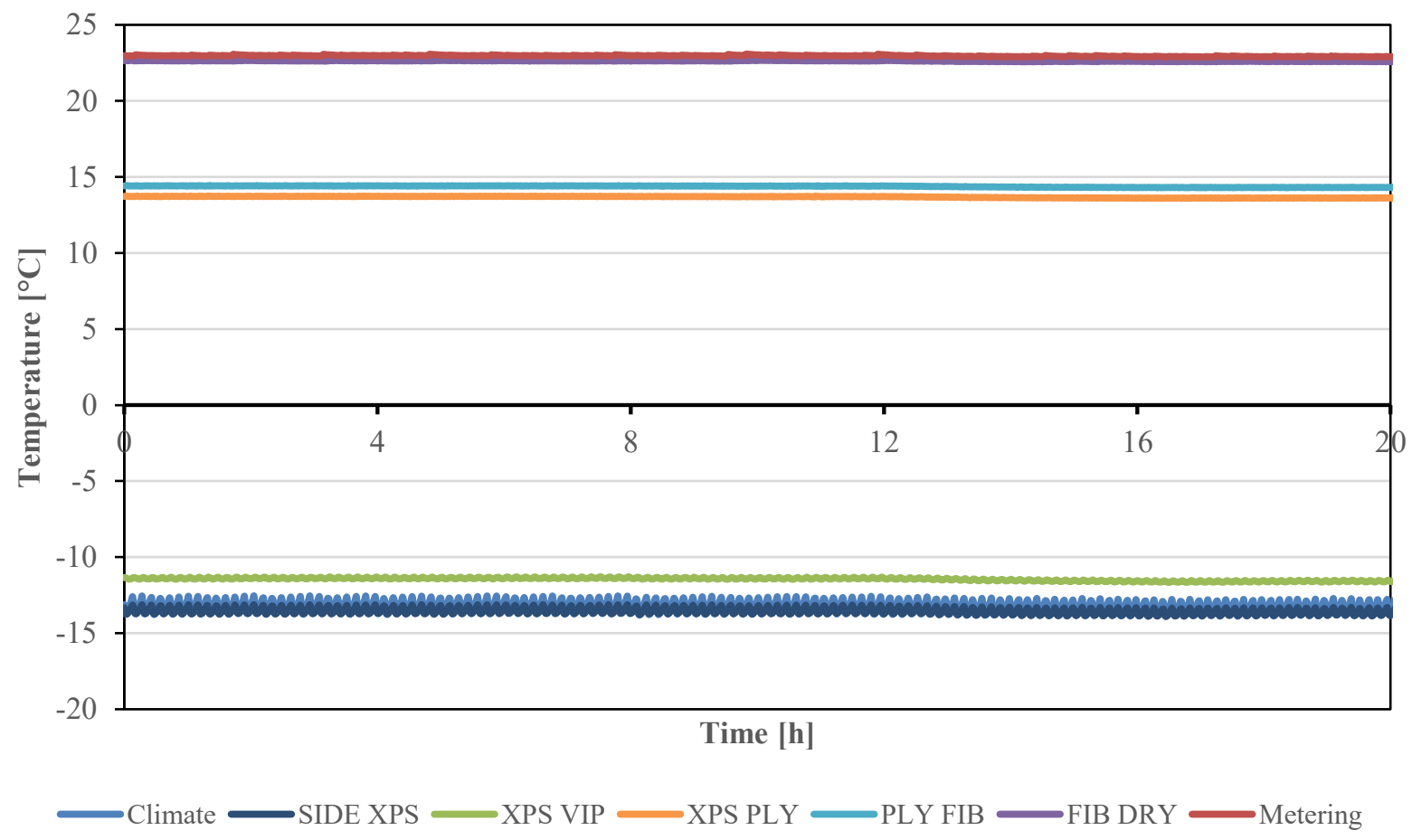

Figure 5-13: Steady-state temperature profile at the VIP-cavity cross section of Test 1 


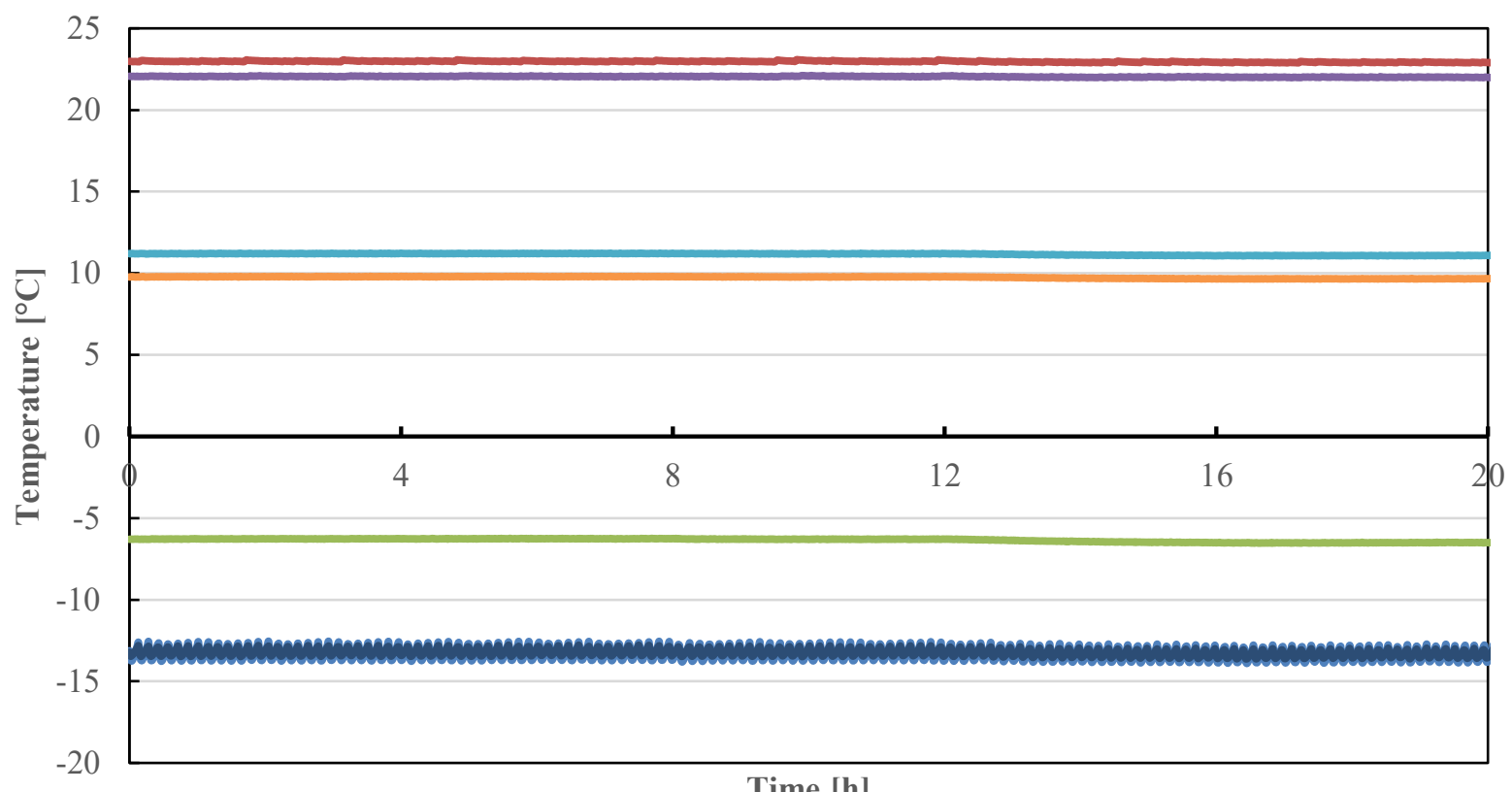

Time $[\mathrm{h}]$

$\longrightarrow$ Climate $\longrightarrow$ SIDE XPS $\longrightarrow$ XPS VIP $\longrightarrow$ XPS PLY $\longrightarrow$ PLY FIB $\longrightarrow$ FIB DRY $\longrightarrow$ Metering

Figure 5-14: Steady-state temperature profile at the XPS-stud cross section of Test 1

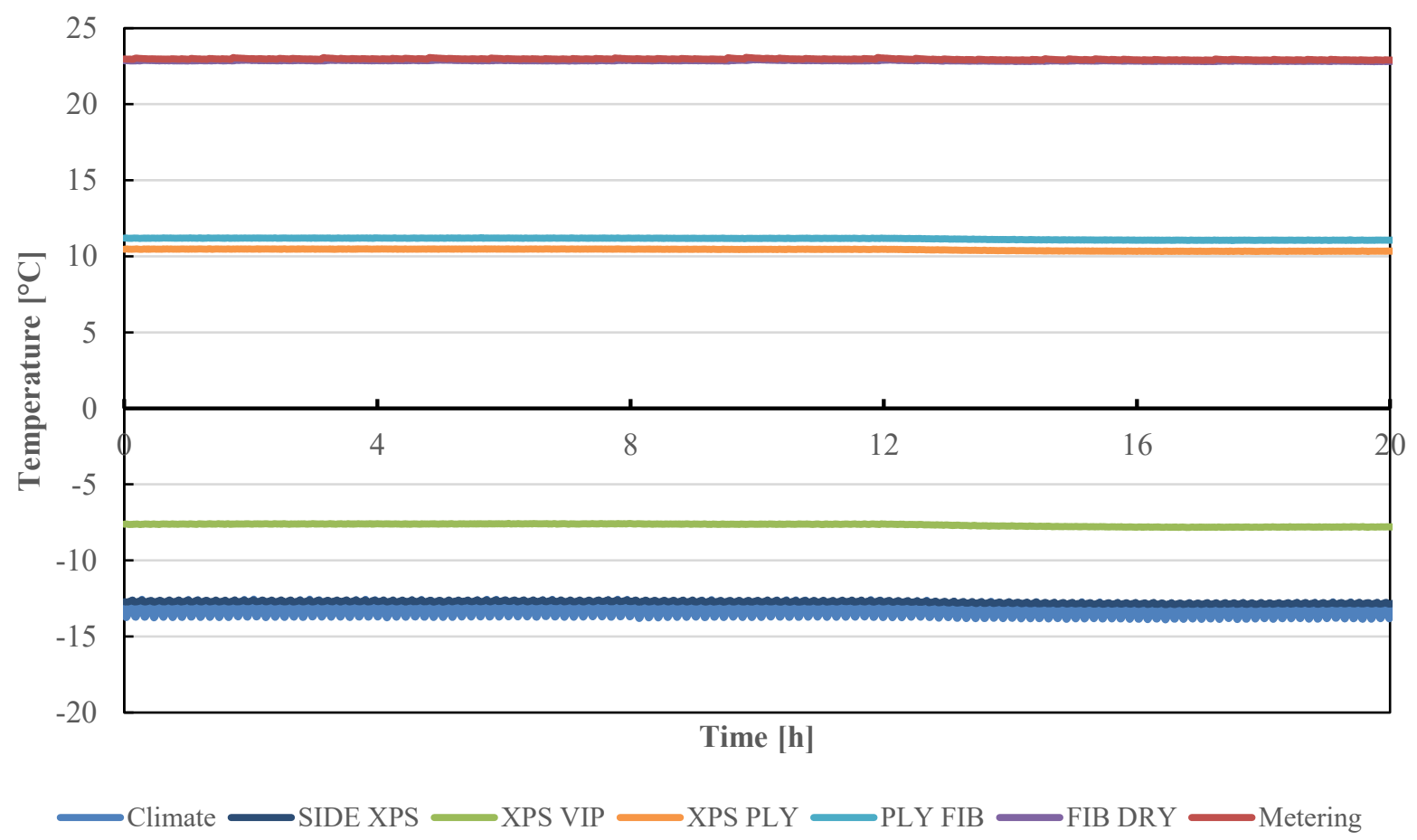

Figure 5-15: Steady-state temperature profile at the XPS-cavity cross section of Test 1 


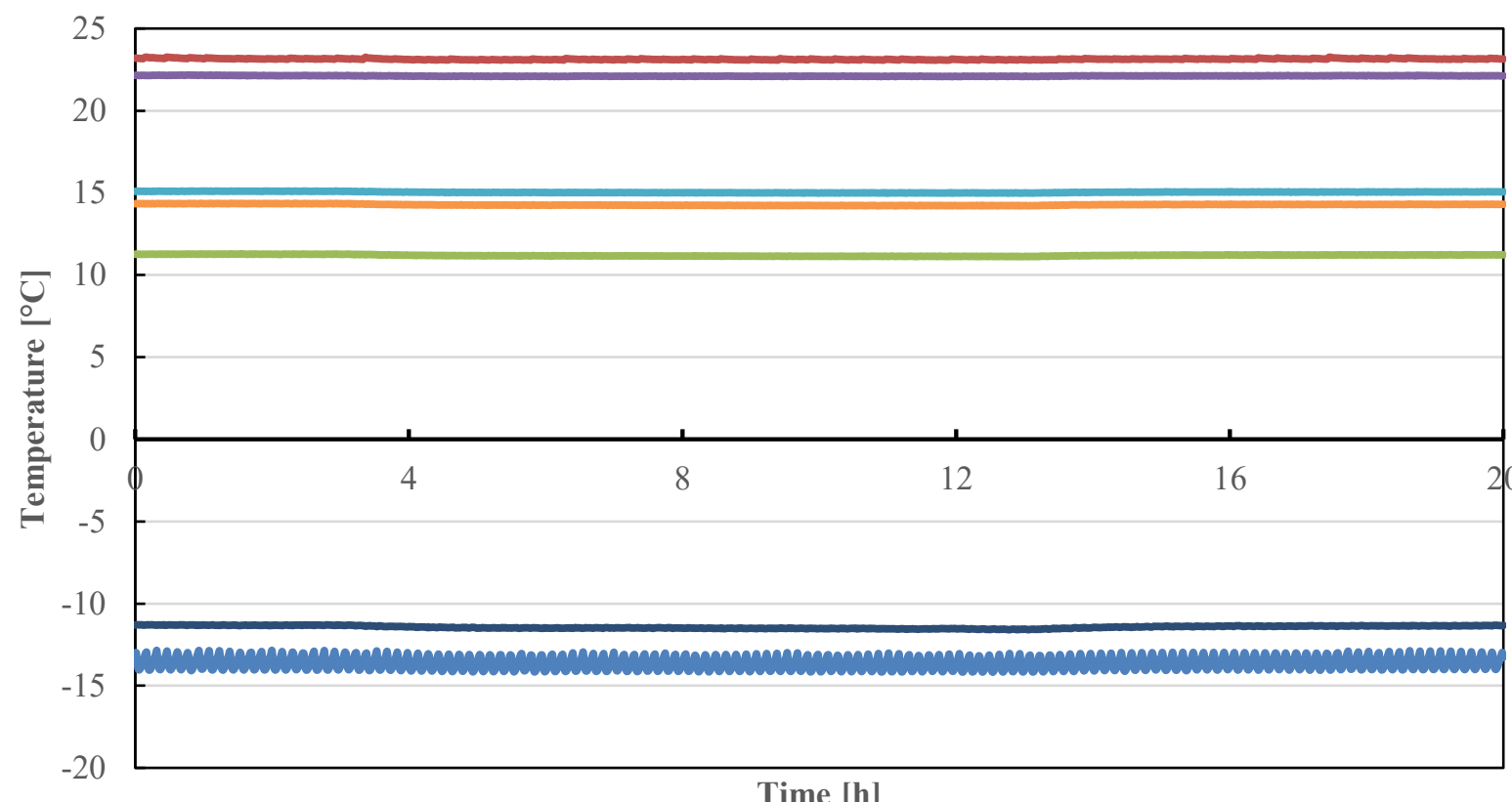

Time $[\mathrm{h}]$

Climate $\longrightarrow$ SIDE XPS $\longrightarrow$ VIP XPS $\longrightarrow$ XPS PLY $\longrightarrow$ PLY FIB $\longrightarrow$ FIB DRY $\longrightarrow$ Metering

Figure 5-16: Steady-state temperature profile at the VIP-stud cross section of Test 2

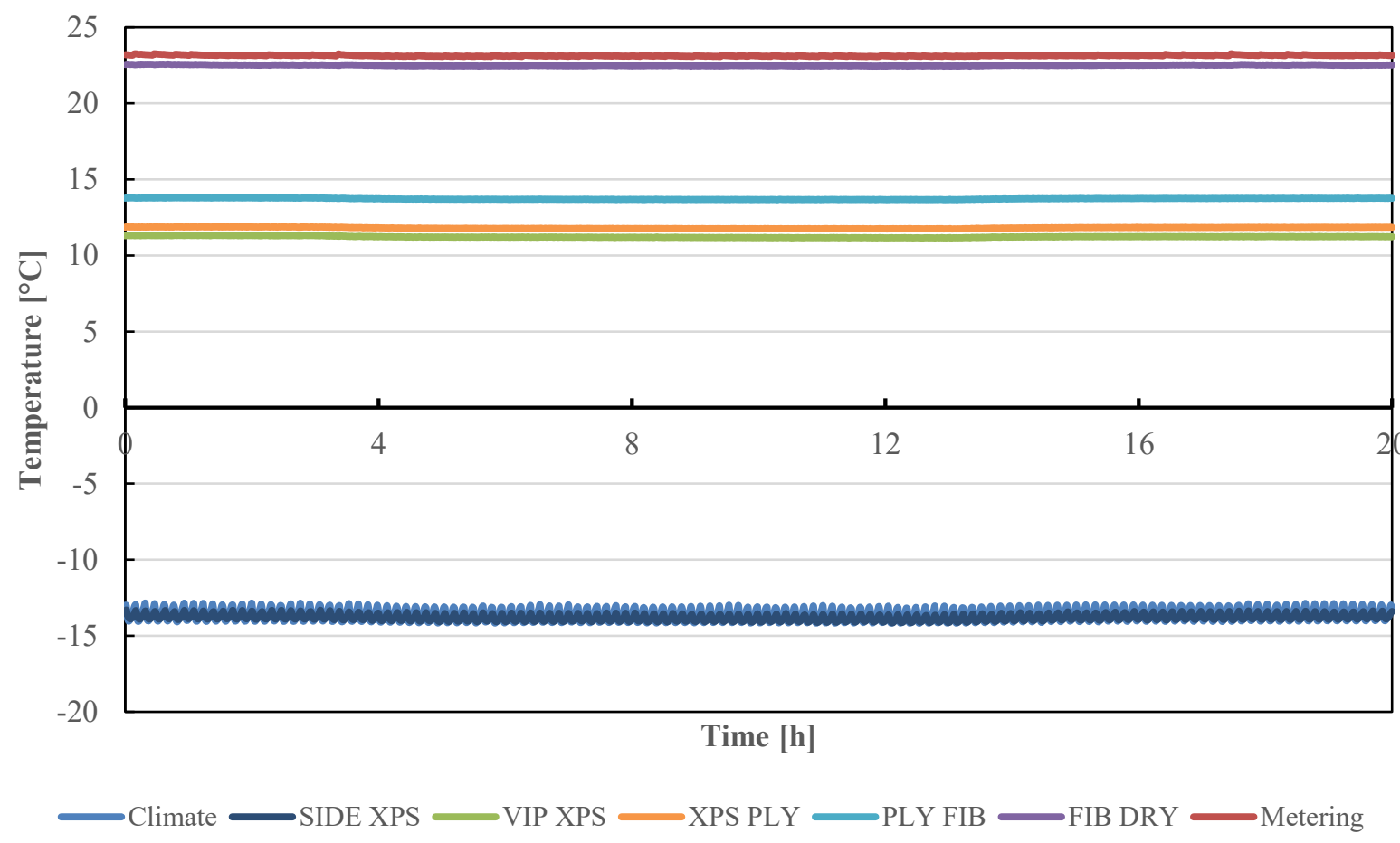

Figure 5-17: Steady-state temperature profile at the VIP-cavity cross section of Test 2 


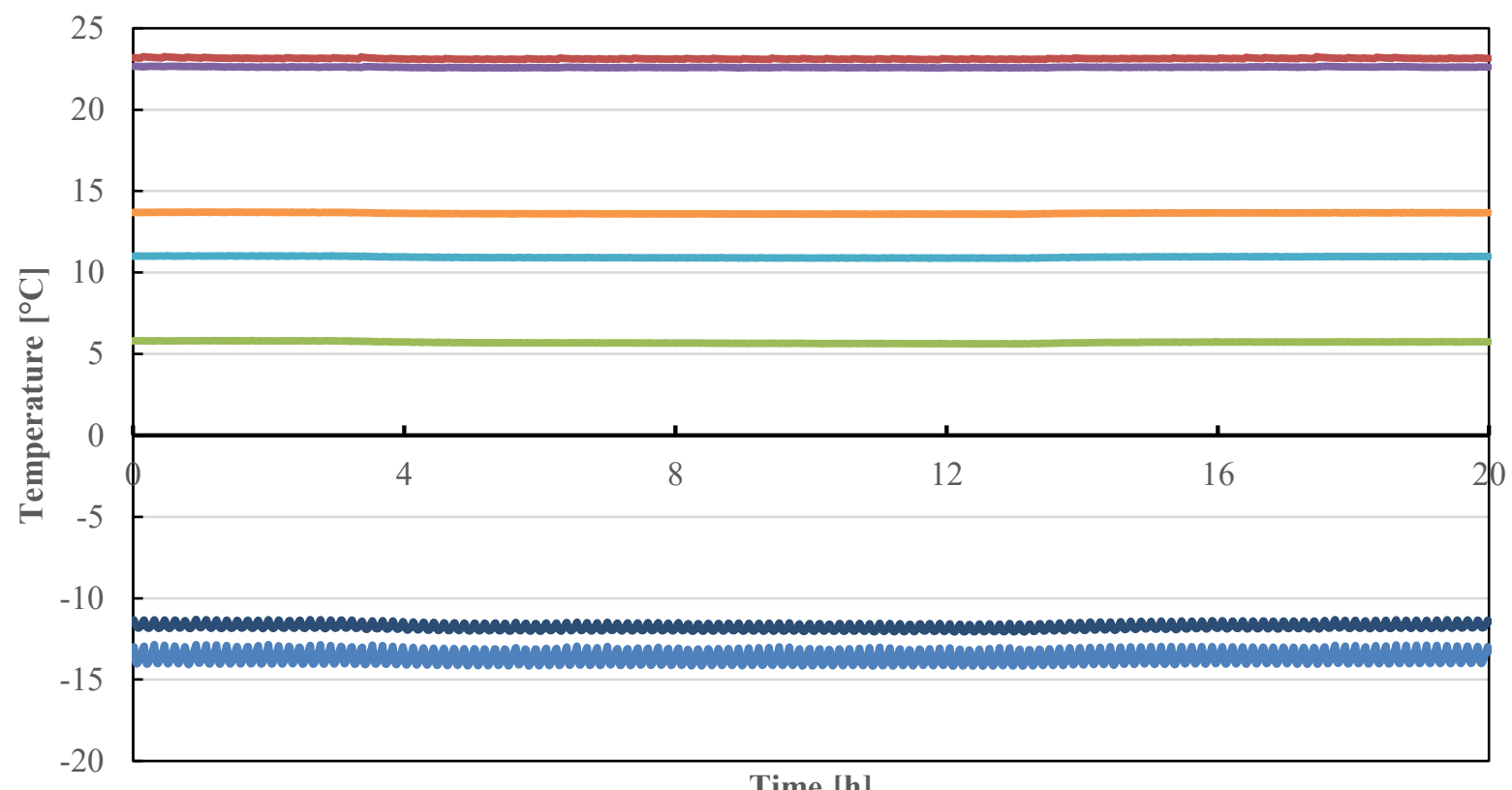

Time $[\mathrm{h}]$

$\longrightarrow$ Climate $\longrightarrow$ SIDE XPS $\longrightarrow$ VIP XPS $\longrightarrow$ XPS PLY $\longrightarrow$ PLY FIB $\longrightarrow$ FIB DRY $\longrightarrow$ Metering

Figure 5-18: Steady-state temperature profile at the XPS-stud cross section of Test 2

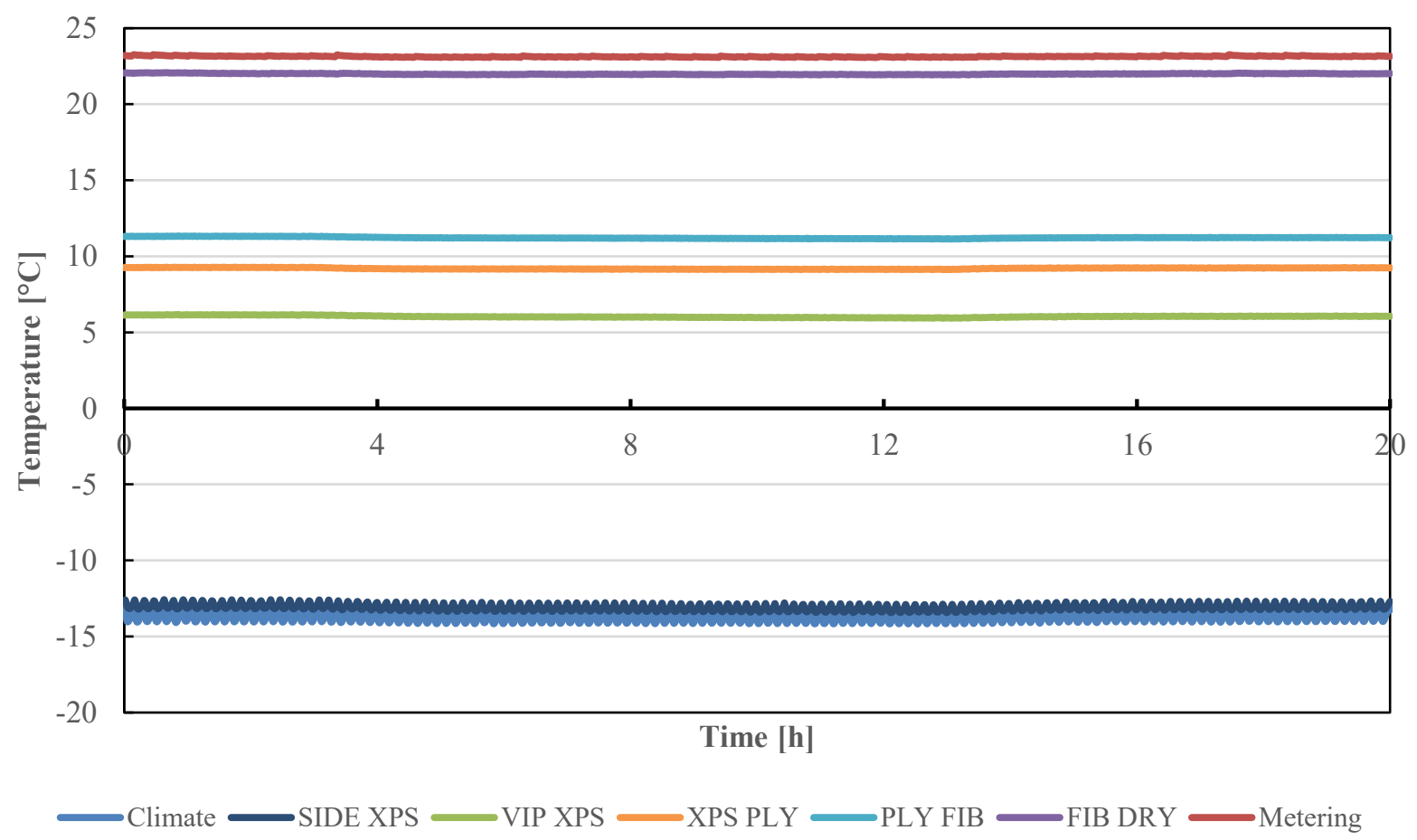

Figure 5-19: Steady-state temperature profile at the XPS-cavity cross section of Test 2 
Since the thermal resistance is a function of the temperature difference across a material, the steady-state graphs show a material's contribution to the effective thermal resistance. The small temperature difference across the plywood shows that it does not enhance the thermal performance of the wall assembly, which can be seen in any of the previous steady-state figures. In contrast, the temperature across the XPS is larger. This is an indicator that it provides better thermal performance. It would be expected that the largest temperature difference would be found across the VIP, which cannot be seen in the Figure 5-12 to Figure 5-19, but instrumentation was embedded in Test 5 to measure the temperatures on the VIP surfaces. They were plotted against time in Figure 5-20. The temperature difference across the VIP was approximately $21^{\circ} \mathrm{C}$ on average through the measurement period. This was much greater than the temperature difference of $4^{\circ} \mathrm{C}$ across the $12.7 \mathrm{~mm}\left(0.5^{\prime \prime}\right) \mathrm{XPS}$ or $10^{\circ} \mathrm{C}$ across the fiberglass insulation used to fill the wall cavities between studs, which can be seen in the steady-state plots from Test 1 and 2 .

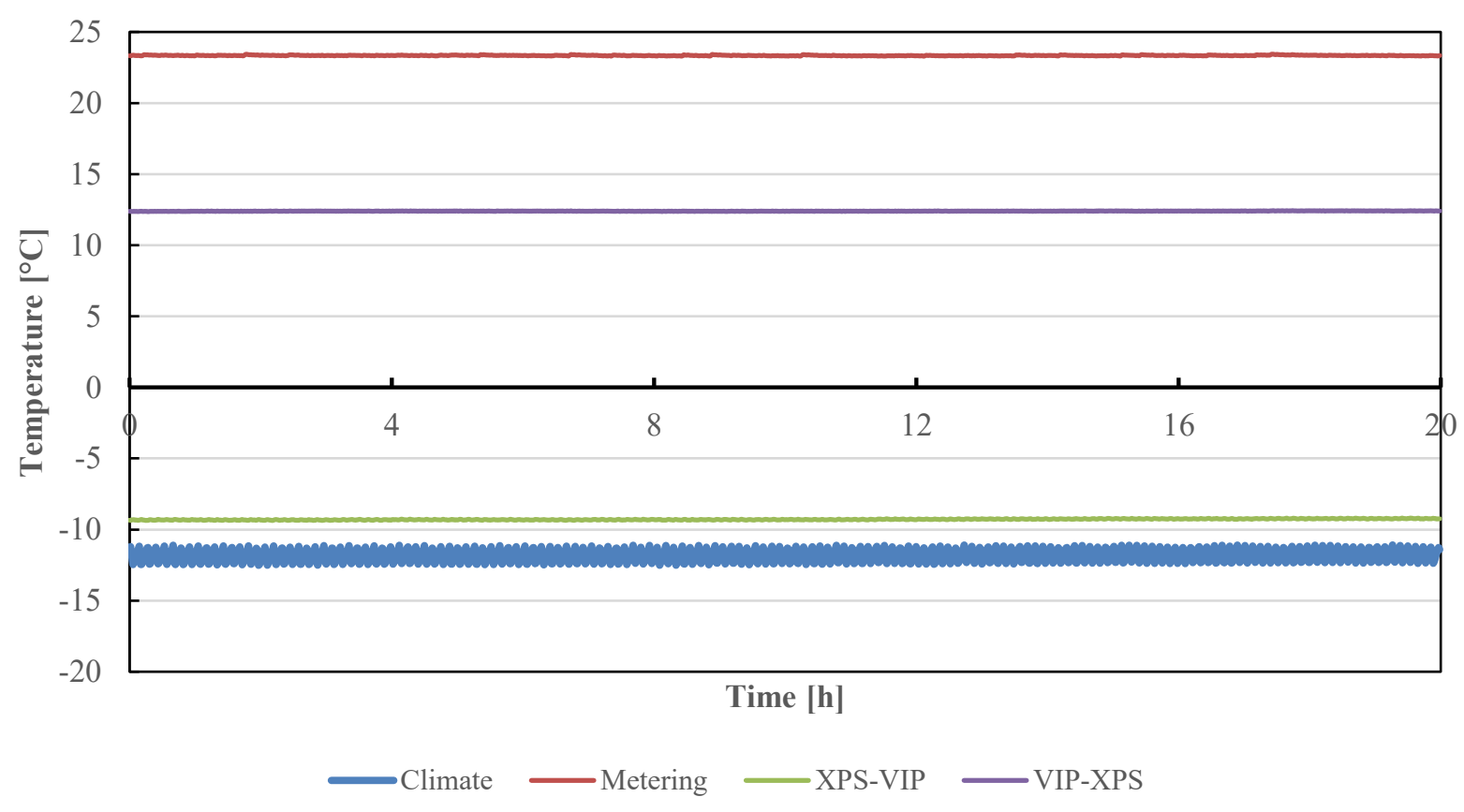

Figure 5-20: Steady-state temperature profile at the VIP-cavity cross section of Test 5 
A number of observations were made from the isotherm profiles; specifically, observations were made on the interior wall surface temperature and the effect from the XPS spacers and wood studs. Since the wood studs are in contact with the drywall, which contains no significant thermal performance compared to other materials used in the wall construction, each wall has a lower temperature at the surface. At the stud location, it did not matter whether it aligned with VIP or XPS. A temperature reading between approximately $19^{\circ} \mathrm{C}$ and $16.5^{\circ} \mathrm{C}$ existed for every wall design. However, when the interior surface was aligned with the cavity, a temperature above approximately $19^{\circ} \mathrm{C}$ was achieved but the XPS spacers affected the surface temperature. In Figure 5-23 and Figure 5-24, the temperature along the surface was consistent and did not deviate much from one cavity, to another. In the remaining isotherms Figure 5-21, Figure 5-22 and Figure 5-25, the XPS affected the shape of the warm isotherms. In Figure 5-23 to Figure 5-25, the XPS caused the temperature to go below the $19^{\circ} \mathrm{C}$ threshold, a temperature within the range caused by the studs. All of these variations are due to the extreme changes in thermal resistance that the wall assembly experiences and these profiles should be a consideration during the design process due to potential dew points and the thermal bridges caused by the materials with lower RSI-values.

\subsection{Modelling Results}

The experimental results were used to verify the modelling approaches for evaluating wall assemblies with 3D heat profiles in 2D heat transfer software. As previously discussed, the VIPs have a distinct change in thermal performance along the face of the panel. However, using a center of panel value or an effective thermal resistance value could be an effective way to evaluate the materials in the model when evaluating a full wall assembly. Since the VIP perimeter is encased in XPS for protection purposes, the XPS creates a change in thermal resistance at multiple points 
in the wall assembly. Therefore, the center or effective value of the VIP is used as the properties for the VIPs in the simulations.

With the XPS acting as a strong thermal bridge, it was necessary to break down the wall assembly into multiple cross sections and use weighted averages based on the height coverage. The modelling was further developed to investigate the effects that reducing the cross sectional area to a single 2D model that could represent the entire main wall.

\subsubsection{Multiple Profile Simulation}

The THERM simulations of the composite panels were performed with a $2 \mathrm{D}$ section including VIPs with XPS and one only with XPS to represent the two 2D sections located in the wall assembly. As mentioned previously, the thermal conductivities of most materials and boundary conditions were found within the THERM architectural library, and when materials were not provided, the material properties were taken from manufacturer's data sheets. The geometries of each cross section including VIPs were outlined in Table 3-3. To calculate the effective RSI-value for each design, seven cross sections were created. These include five containing VIPs and two without VIPs. Only two cross sections without VIPs were required since the only variance between designs was the stud spacing.

Using the THERM simulations, the RSI-value of the 2D sections was found and the effective RSI-value of the wall designs was computed. The coverage of each 2D section was used as the averaging parameter since the modelled geometries encompassed the horizontal properties of the wall assembly. As such, the wall assembly varied in one direction, which was the vertical height of the wall. The height coverage for each $2 \mathrm{D}$ section on a $2.5 \mathrm{~m}(8 \mathrm{ft}$.) wall was obtained from construction diagrams and measurements during installation. The height ratio is the total coverage height for the 2D section in meters divided by the total height $(2.5 \mathrm{~m})$. Table $5-6$ contains 
the results from THERM simulations of each wall design, including height ratio and the individual 2D section RSI-values that are averaged to determine the effective RSI-values of the wall designs.

Table 5-6: Summary of THERM results using weighted average technique

\begin{tabular}{|c|c|c|c|c|c|}
\hline & 2D Section & $\begin{array}{c}\text { RSI } \\
{\left[\mathrm{m}^{2} \mathrm{~K} / \mathrm{W}\right]}\end{array}$ & $\begin{array}{c}\text { Height } \\
{[\mathrm{m}]}\end{array}$ & Height Ratio & Weighted RSI $\left[\mathrm{m}^{2} \mathrm{~K} / \mathrm{W}\right]$ \\
\hline \multirow[t]{3}{*}{ Test 1} & VIP with XPS & 8.67 & 2.3 & 0.94 & 8.13 \\
\hline & XPS & 4.35 & 0.2 & 0.06 & 0.27 \\
\hline & & & & Effective RSI & 8.40 \\
\hline \multirow[t]{3}{*}{ Test 2} & VIP with XPS & 9.27 & 2.2 & 0.92 & 8.50 \\
\hline & XPS & 4.48 & 0.2 & 0.08 & 0.37 \\
\hline & & & & Effective RSI & 8.87 \\
\hline \multirow[t]{3}{*}{ Test3 } & VIP with XPS & 6.92 & 2.0 & 0.82 & 5.66 \\
\hline & XPS & 4.48 & 0.4 & 0.18 & 0.82 \\
\hline & & & & Effective RSI & 6.48 \\
\hline \multirow[t]{3}{*}{ Test 4} & VIP with XPS & 9.25 & 2.0 & 0.82 & 7.56 \\
\hline & XPS & 4.48 & 0.4 & 0.18 & 0.82 \\
\hline & & & & Effective RSI & 8.38 \\
\hline \multirow[t]{3}{*}{ Test 5} & VIP with XPS & 8.90 & 2.3 & 0.94 & 8.34 \\
\hline & XPS & 4.48 & 0.2 & 0.06 & 0.28 \\
\hline & & & & Effective RSI & 8.62 \\
\hline
\end{tabular}

The modelling results also provided isotherms through the wall assembly. The isotherms illustrate the temperature gradients through the wall assembly, from warm to cold surfaces as well as the difference in temperature between coverage of VIPs versus XPS. The isotherms have the temperatures labelled in addition with a red (hot) to blue (cold) colour gradient between the lines to visualize the temperature change within the assembly. Figure 5-21 to Figure 5-25 includes the THERM cross sections (top) from Test 1 to Test 5 and the isothermal profiles (bottom). The perimeter of the VIP was outlined in a bolded, darker line on top and the isotherms were included in the drawing below. 
Interior

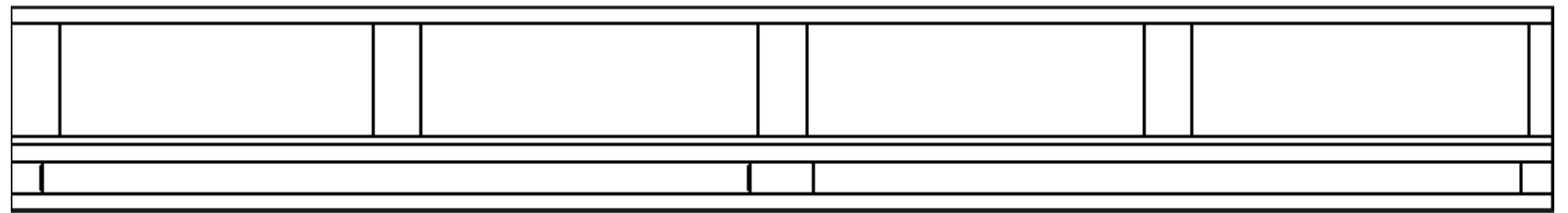

Exterior

Interior

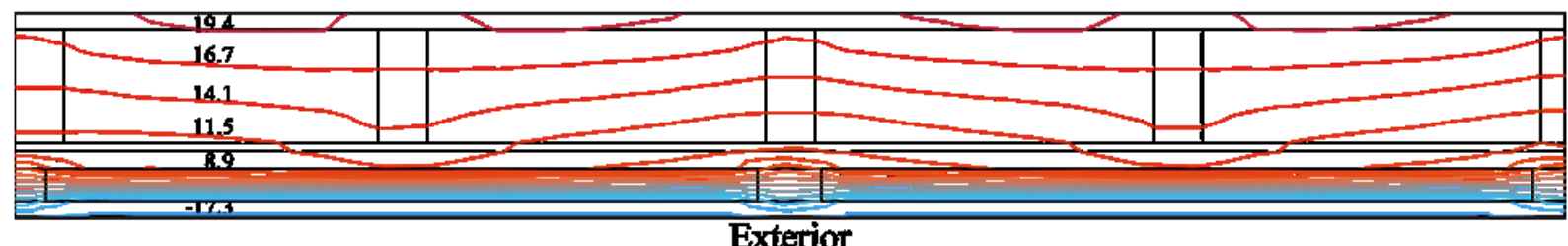

Exterior

Figure 5-21: Test 1 cross section incorporating VIPs (top) and isotherm profiles (bottom)

Interior

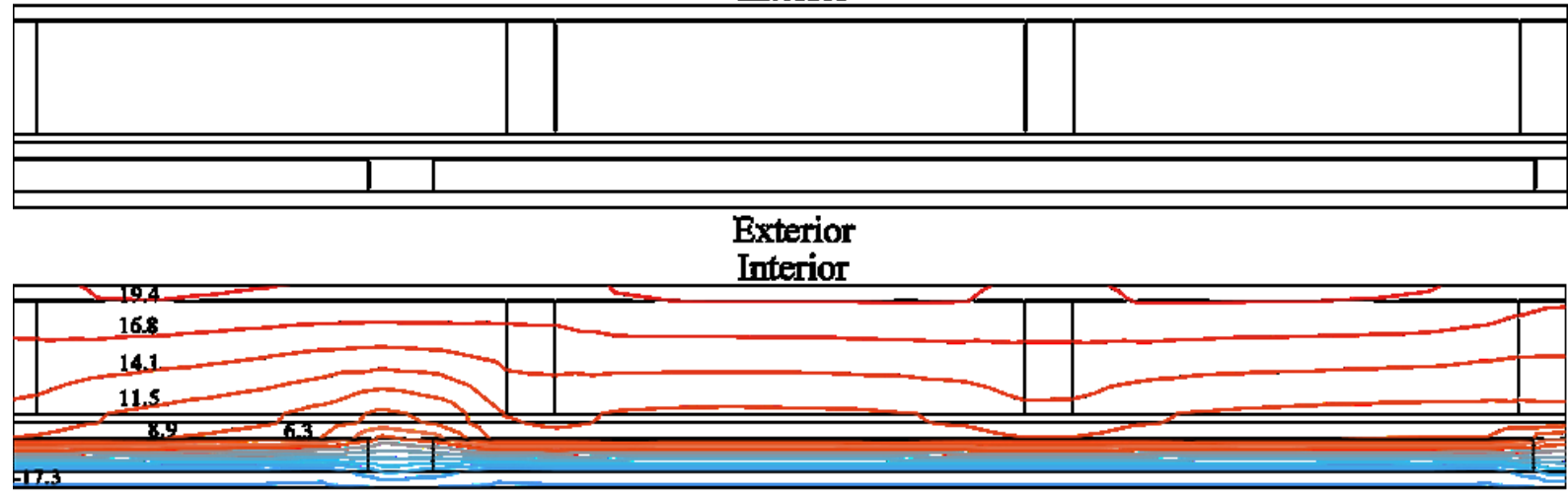

Exterior

Figure 5-22: Test 2 cross section incorporating VIPs (top) and isotherm profiles (bottom)

Interior

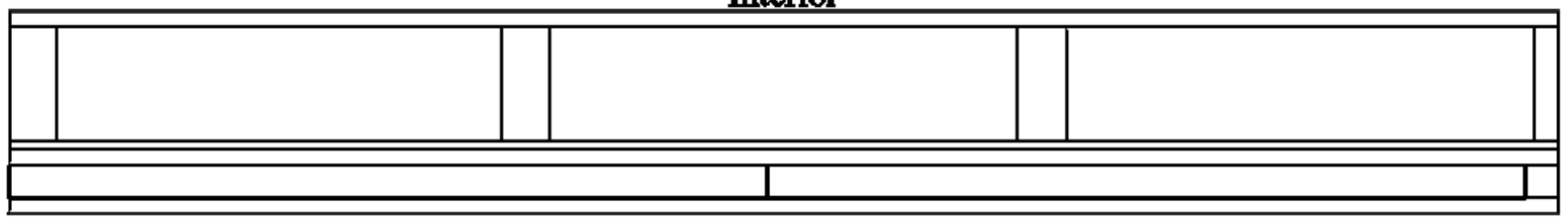

Exterior

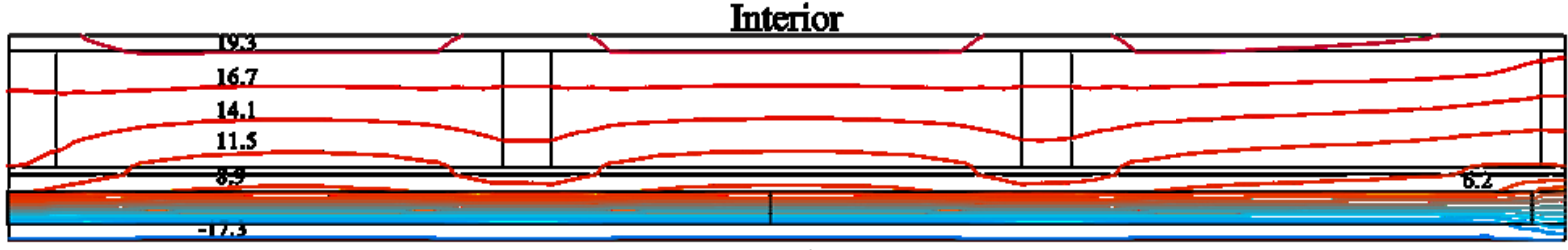

Exterior

Figure 5-23: Test 3 cross section incorporating VIPs (top) and isotherm profiles (bottom) 


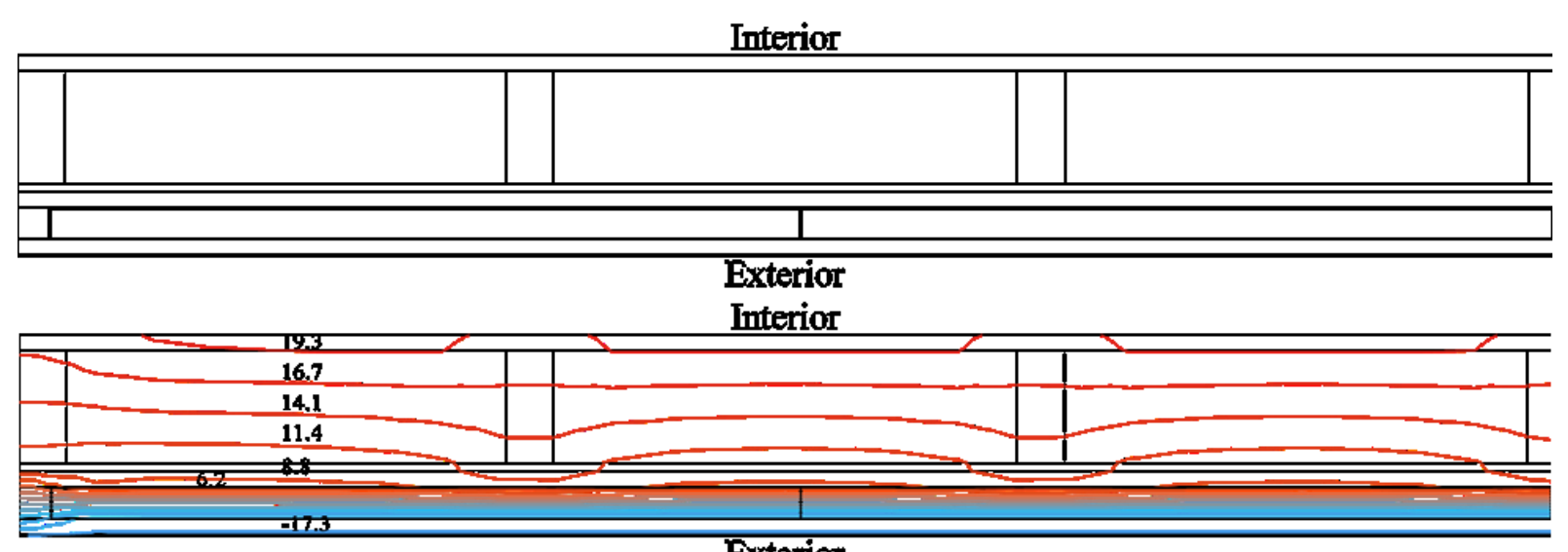

Exterior

Figure 5-24: Test 4 cross section incorporating VIPs (top) and isotherm profiles (bottom)

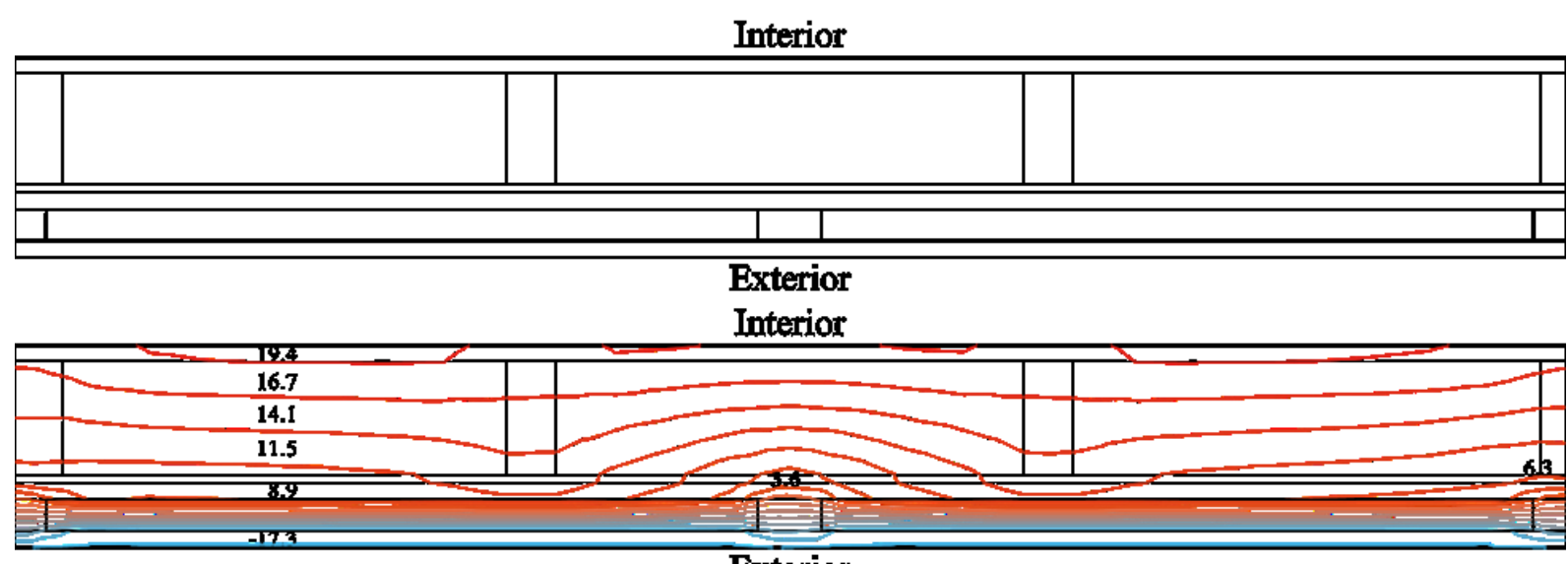

Exterior

Figure 5-25: Test 5 cross section incorporating VIPs (top) and isotherm profiles (bottom)

The modelling study produced the effective RSI-values of each wall section as well as the isotherms through the VIP cross section. It is clear in the profiles that a large temperature variation existed within the wall, but dissipated at the interior wall surface. In Figure 5-21, the center wood stud aligns with the XPS spacer and causes a wide temperature gradient since the distance between the isotherm lines is large. This outcome was expected since it was the point where the lowest thermal resistance would exist within the wall designs. While this point was be used for mechanical fastening, a large heat loss and thermal bridge existed there. Also in Figure 5-21, the isotherms through the VIP and wall cavity section shows a steep temperature gradient across the VIP, from 
high thermal resistance. That wall contains the highest and lowest possible linear thermal resistances achievable in the wall project designs and the isotherms effectively show the change in temperature caused within the wall.

\subsubsection{Single Profile Method for Non-Homogenous Wall Assemblies}

It was desired to condense the multiple unique 1D cross sections into a single, fictitious representative cross section based on material coverage ratio that could be made to replicate properties in a representative geometry. Using common simplification techniques performed in wall assembly simulations, creating two fictitious studs that represent the amount of VIPs or XPS stud coverage was necessary. The single cross section included a fictitious size of XPS and VIP based on the coverage area of the materials, such that the model geometry is identical to Figure 4-4. This was done as opposed to creating a single fictitious stud at the center of the model geometry in order to simplify the comparison process of coverage areas between various designs.

With the two studs and materials separated, the changes in stud and material coverage, and temperature variation within the assembly through isotherms are better visualized and more apparent. The coverage areas of the $2 \mathrm{D}$ cross section for each wall design is contained in Table 5-7 and the ratio of cross section coverage to overall height was used to determine the composition of the new models. The material properties and boundary conditions were kept from the initial multi cross section simulations previously performed and as such, the results from the simulations were tabulated into Table 5-7 as well. 
Table 5-7: Coverage area and results for each cross section in single profile simulation

\begin{tabular}{l|ccccc}
\hline Test & $\begin{array}{c}\text { VIP Cavity } \\
{[\mathrm{mm}]}\end{array}$ & $\begin{array}{c}\text { XPS Cavity } \\
{[\mathrm{mm}]}\end{array}$ & $\begin{array}{c}\text { VIP Stud } \\
{[\mathrm{mm}]}\end{array}$ & $\begin{array}{c}\text { XPS Stud } \\
{[\mathrm{mm}]}\end{array}$ & $\begin{array}{c}\text { RSI-value } \\
{\left[\mathrm{m}^{2} \mathrm{~K} / \mathrm{W}\right]}\end{array}$ \\
1 & 1047 & 171 & 92 & 78 & 8.38 \\
2 & 1047 & 171 & 87 & 45 & 8.62 \\
3 & 732 & 487 & 46 & 86 & 6.53 \\
4 & 976 & 243 & 93 & 40 & 7.76 \\
5 & 1047 & 171 & 64 & 68 & 8.47 \\
\hline
\end{tabular}

After using the single profile to model the building envelope, effective RSI-values were comparable to the values obtained from the multiple profile method. The isothermal profiles were taken from the THERM simulations and provided insight into the results. The isotherm profile for Test 1 through Test 5, in Figure 5-26 to Figure 5-30, shows the temperature gradients through the assembly as well as the effects that aligning the stud with VIP versus XPS caused. By having the XPS cover more of the stud, seen in the Figure 5-26 to Figure 5-30 or through the coverage areas in Table 5-7, the composite panel allowed greater constructability but saw a significant decrease in effective RSI-value. The RSI-value from the XPS aligned with the stud was predominant in Figure 5-28 which was also seen during the multiple cross section simulation. The envelope design where the most area of XPS aligned with the stud and maintained an effective above RSI $7.04 \mathrm{~m}^{2} \mathrm{~K} / \mathrm{W}$ appeared to be Test 5. The VIP and XPS were aligned with the stud the same amount, while yielding a high RSI-value building envelope, well above the intended goal.

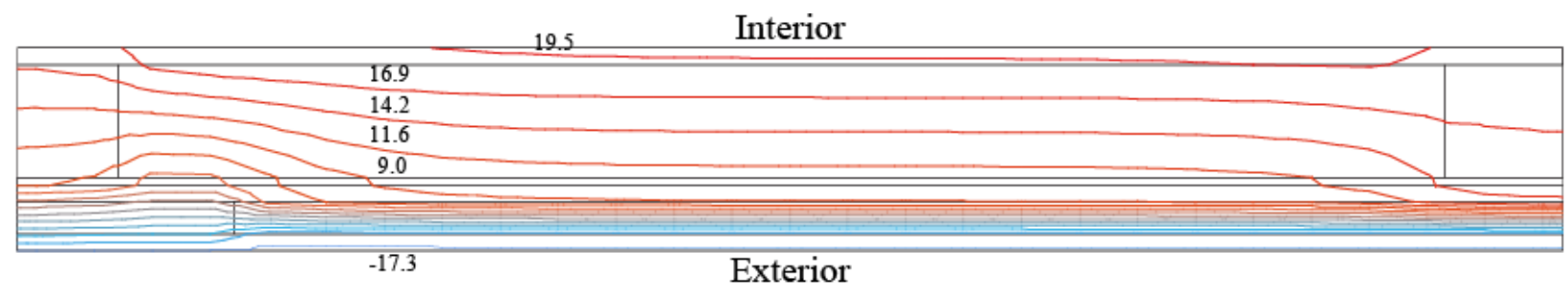

Figure 5-26: Test 1 single cross section isotherm profile 


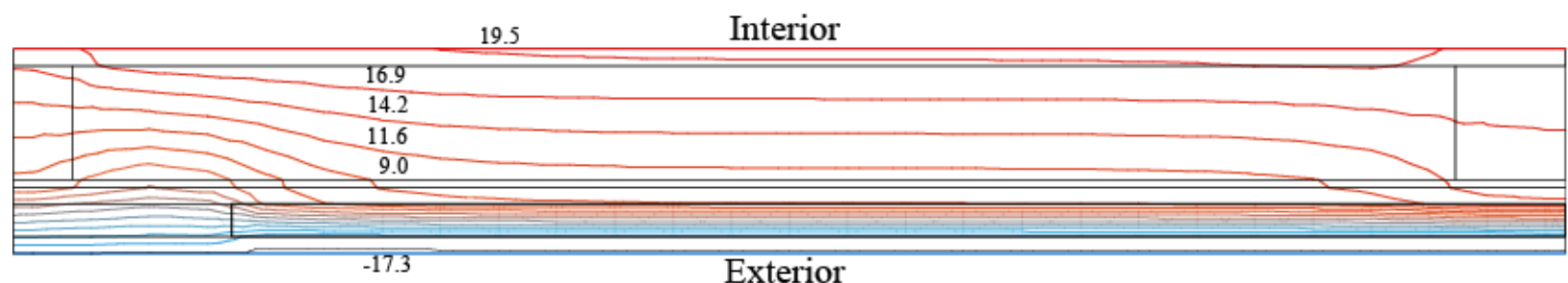

Figure 5-27: Test 2 single cross section and isotherm profile

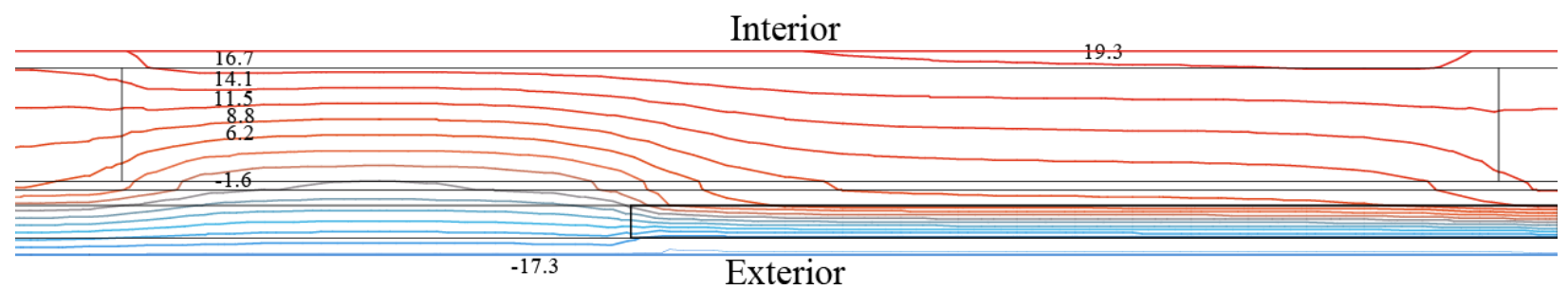

Figure 5-28: Test 3 single cross section isotherm profile (bottom)

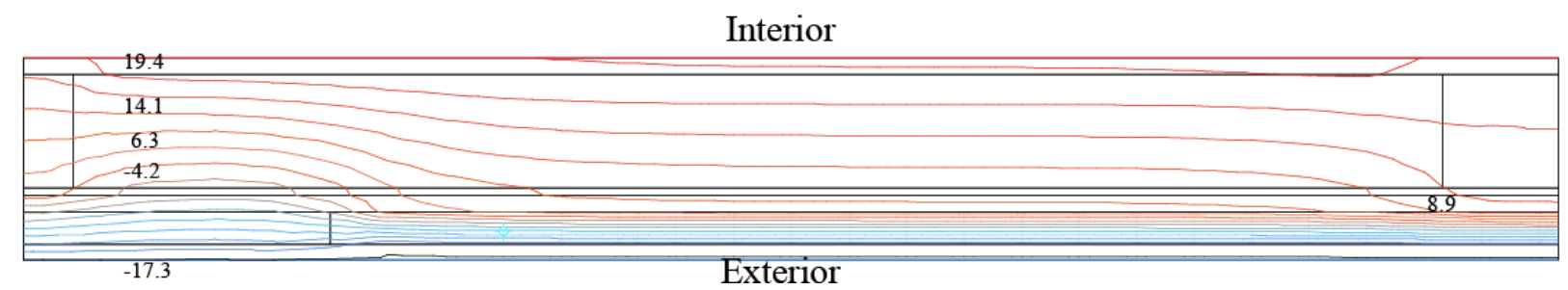

Figure 5-29: Test 4 single cross section isotherm profile

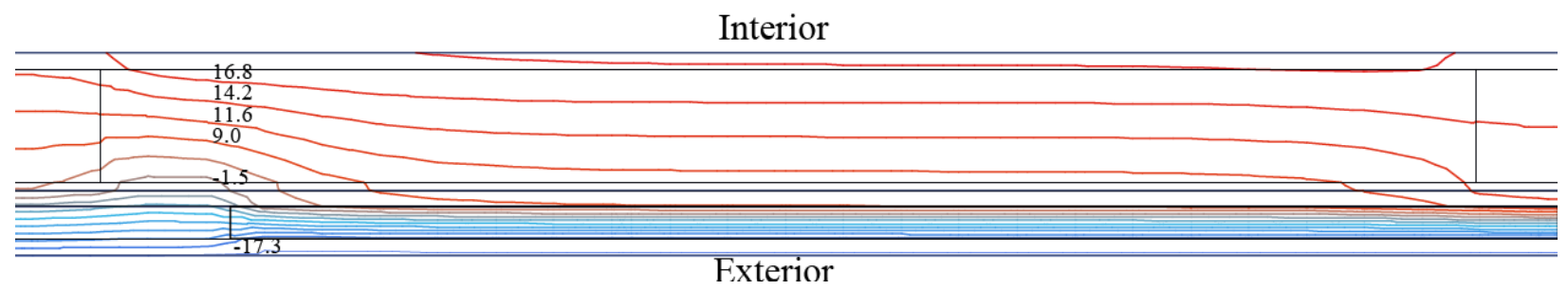

Figure 5-30: Test 5 single cross section isotherm profile

\subsection{Summary}

This chapter presented the experimental and computer generated thermal performance results for VIPs and high RSI-value building envelopes. The guarded hot-box was used to evaluate the effective RSI-values of single and double layered VIP assemblies, as well as the changes in 
thermal resistance throughout the assemblies. A 32\% reduction in RSI-value between the center and edge of a VIP was observed. After tiling the second layer of VIPs to the center-corner offset, the difference maximum, and minimum heat flux was approximately 5\%, the smallest of the three offsets. The composite insulation panel with VIPs on a wood framed wall was evaluated using the guarded hot-box and varied with a RSI-value between $9.07 \mathrm{~m}^{2} \mathrm{~K} / \mathrm{W}$ and $8.44 \mathrm{~m}^{2} \mathrm{~K} / \mathrm{W}$. The wall designs were also modelled in THERM using multiple profiles and a single profile. Modelling the wall assembly using multiple cross sections and using an area-weighted average produced effective RSI-values between $6.48 \mathrm{~m}^{2} \mathrm{~K} / \mathrm{W}$ to $8.87 \mathrm{~m}^{2} \mathrm{~K} / \mathrm{W}$. When using a single cross section to model the building, the RSI-values were $6.53 \mathrm{~m}^{2} \mathrm{~K} / \mathrm{W}$ to $8.62 \mathrm{~m}^{2} \mathrm{~K} / \mathrm{W}$.

The next chapter discusses the impact of heat flux variations caused by VIPs, the overall performance and applicability of VIPs in building envelopes and a comparison between the two modelling methods. Finally, the uncertainty analysis of the guarded hot-box is provided. 


\section{Chapter: Discussion}

The experimental and modelling results provided insight into the unknown aspects of VIPs and their integration into building envelope construction. The edge effects that can hinder the thermal performance were analyzed, and quantified. The existing temperature gradient due to the non-homogenous properties of the VIPs was visualized using capturing infrared pictures and creating heat maps. The effects of single and double layer configurations on the envelope were discussed. In addition, it was observed that different VIP orientations would change the heat flux through the assembly, specifically at the VIP-VIP interface.

The composite insulation panels with VIPs were evaluated on a full wall assembly for their effective thermal resistance and constructability. The management of XPS and VIP coverage areas were important to the overall desired design properties such as thermal performance, constructability and VIP protection. Five different composite panels were evaluated at steady-state conditions and modelled to find the temperature gradients and effective RSI-values.

The non-homogenous properties of the VIP and composite panel construction led to the development of two new modelling techniques to properly evaluate the building envelopes. A novel iterative approach was developed to separate and assign different material properties to the edges and centers of VIPs using 2D heat transfer software since experimental work concluded that a large discrepancy exists between the center and edge of the panel. A second method was developed which was based on creating a single representative cross section using the coverage area ratios of materials to ease the creation and comparison of main wall assemblies in THERM and validated against steady-state experimental data. Finally, an uncertainty analysis of the experimental data and results from the guarded hot-box steady-state testing and the THERM computer modelling was performed. 


\subsection{Heat Flux Variation in VIPs}

The ability to instrument the VIPs with heat flux meters and temperature sensors allowed for detailed data collection. The manufacturer specifications sheet provided the center of panel RSI-value even though a significant loss occurs at the edge. While some products have begun including effective panel values that are closer to realistic performance, it is desirable to quantify the amount of reduced heat flux between the edge and center. Thermal images offered a qualitative measurement of the change in heat flux through reading the surface temperatures at the interface but the embedded heat flux plates provided a quantitative measurement applicable to other types of VIPs.

During the evaluation of a single layer of VIPs at steady-state conditions, the experimental results showed that there was a $32-36 \%$ reduction in RSI-value existed between the center and edge values on a single layer and that, the effective RSI-value depended on the length of edge versus the surface area of the panel. The results allowed for significant insight to the variance in performance through a single panel and led to further evaluation of the modelling approach. Creating an iterative edge approach to modelling building envelopes incorporating VIPs provided a more accurate representation of the computer and experimental results. By separating the VIP edge and center into two different materials within THERM, a difference between the simulated results was reduced from $4.7 \mathrm{~m}^{2} \mathrm{~K} / \mathrm{W}$ to $4.5 \mathrm{~m}^{2} \mathrm{~K} / \mathrm{W}$. The model becomes more realistic by separating the edges into smaller sections, such as two $25.4 \mathrm{~mm}$ (1") sections with thermal conductivities of $0.0041 \mathrm{~W} / \mathrm{m} \cdot \mathrm{K}$ and $0.0063 \mathrm{~W} / \mathrm{m} \cdot \mathrm{K}$.

Modelling the VIPs by assigning an effective RSI-value, which encompassed the edge effects as opposed to separating the VIP into multiple RSI-values, was found to be a sufficient material property to use in building envelope simulations. However, a limitation in assigning an 
effective RSI-value for the VIP is that the simulated temperature distribution would change due to the thermal bridge from the edges. The methods yield similar effective thermal resistance results, applicable and useful for building simulations. However, the iterative method would provide a better representation of the actual scenario. The temperature distribution through the assembly using the iterative method could reveal potential hygrothermal or other temperature variance induced problems within the building envelope assembly.

\subsection{VIP Offset Effects on Heat Flux and Temperature}

Through carefully designing and managing the alignment of the second layer VIP edges and corners, the temperature distribution and heat flux could be moderated closer to uniformity. The effective RSI from the steady-state test results did not show a significant change in performance. However, there was a strong difference between the heat flux measurements through each unique cross section for each VIP offset wall design. A VIP offset has a significant impact on the maximum and minimum heat flux through the assembly, and the offsets were found to be very successful at creating a more homogenous layer. When the VIP was not offset, which was the baseline case, the difference between the highest (corner-corner) and lowest (center-center) was experimentally found to be approximately $35 \%$. Conceptually, this would yield the highest difference due to aligning the highest (center-center) and lowest (corner-corner) thermal resistant cross sections.

When the VIPs were shifted horizontally to align the VIP center of the second layer to the VIP seam of the first layer, a limited favorable change in heat flux variation was found. The percent difference between the maximum and the minimum was about $25 \%$. The small change in heat flux difference is due to the limited difference in thermal resistance between the seam and corner of the VIPs. Previous tests found that the RSI-value of a corner was $1.5 \mathrm{~m}^{2} \cdot \mathrm{K} / \mathrm{W}$ while the seam was 
RSI-1.6 $\mathrm{m}^{2} \cdot \mathrm{K} / \mathrm{W}$. The final orientation evaluated was offsetting the second layer's VIP center to the VIP corner of the first layer. The final orientation believed to yield the most effective means of limiting heat flux variations, and experimental data supported the expectations. A large change between maximum and minimum was found to be approximately $5 \%$, which was a much more uniform heat flux than the other two orientations. The data from the steady-state testing, shown in Figure 5-8, illustrates the difference between the highest and lowest heat flux as a percentage during the length of the measurement period. The variation in percentages is due to the sensitivity of the heat flux sensor as well as the temperature variation from the cycling of refrigeration equipment.

A qualitative measure to support the changes in heat flux experimental data was provided with infrared photos of the warm side surface temperature. These photos, shown in Figure 5-11, were captured after the completion of the steady-state measurement period. While the colour scales for the images are not the same, the trends do provide valuable insight and validation to the experimental measurements. It conclusively shows that tiling the VIPs so the center covers the corners can create a nearly homogenous insulation layer with nearly homogenous temperature gradients.

For VIPs in the center-center orientation, the perimeter of each VIP is outlined as low temperature regions, denoted by the blue, and is very prevalent in the photo. The center of the VIPs had a higher surface temperature, denoted by the red. By shifting the second layer VIPs to the center-seam orientation, the surface temperature was slightly moderated, but the seams are still apparent. The surface temperatures of the vertical and horizontal seams require the most attention because of the variation. The vertical seam surface temperatures seem to blend appropriately with the center of panel temperature, while below them along the horizontal seam is a subtle drop 
temperature. This temperature difference is appropriate because the top and bottom VIP seams of both layers are aligned compared to aligning the vertical seams with the VIP center. Finally, for the center-corner orientation, the infrared photo shows a nearly uniform temperature distribution. It is clear from the infrared photo that the difference in heat flow through this assembly should be small or minimal compared to the other VIP offsets.

\subsection{Comparison of Evaluation Methods}

The effective RSI-value of the non-homogenous wall assemblies was evaluated through steady-state testing in the guarded hot-box and modelled using THERM by multiple and single cross section methods. The single profile method was developed to provide a solution to creating an appropriate cross section geometry in 2D heat transfer software when $3 \mathrm{D}$ effects occur. The single profile method was compared to the standard modelling method that incorporated multiple cross sections and the weighted average to find the effective RSI-value. The percent difference of each evaluation was compared to the multiple profile modelling method since it contains the expected value based on material's thermal properties. The percent differences between the multiple profile models to the steady-state experimental and single profile model yielded varying results, shown in Table 6-1.

Table 6-1: Percent difference between RSI of designs using various evaluation methods

\begin{tabular}{c|ccccc}
\hline \multicolumn{2}{c}{ Multi-profile $\left(\mathrm{m}^{2} \mathrm{~K} / \mathrm{W}\right)$} & Steady-State $\left(\mathrm{m}^{2} \mathrm{~K} / \mathrm{W}\right)$ & $\%$ Diff. & $\begin{array}{c}\text { Single Profile } \\
\left(\mathrm{m}^{2} \mathrm{~K} / \mathrm{W}\right)\end{array}$ & $\begin{array}{c}\% \\
\text { Diff. }\end{array}$ \\
\hline Test 1 & 8.40 & 8.97 & $6.5 \%$ & 8.38 & $0.2 \%$ \\
Test 2 & 8.87 & 8.97 & $1.1 \%$ & 8.62 & $2.8 \%$ \\
Test 3 & 8.43 & 8.44 & $0.1 \%$ & 6.56 & $22 \%$ \\
Test 4 & 8.38 & 9.07 & $7.9 \%$ & 7.80 & $7.0 \%$ \\
Test 5 & 8.62 & 8.97 & $3.9 \%$ & 8.46 & $1.9 \%$ \\
\hline
\end{tabular}

The discrepancy between the steady-state experimental results and modelling results is found to be between $0.1 \%$ and $7.9 \%$ due to various anticipated factors. The XPS, rated as 
RSI-34.7 $\mathrm{m}^{2} \cdot \mathrm{K} / \mathrm{W}$ per meter thickness $\left(5 \mathrm{ft}^{2 \circ} \mathrm{F} / \mathrm{BTU} \cdot \mathrm{h}\right.$ per inch) and the batt insulation RSI-2.1 $\mathrm{m}^{2} \cdot \mathrm{K} / \mathrm{W}\left(12 \mathrm{ft}^{\circ} \mathrm{F} / \mathrm{BTU} \cdot \mathrm{h}\right)$ effective are the main sources of increases in thermal performance in the wall. However, it is possible that the center of panel thermal conductivity provided by the manufacturer for the VIP materials has a lower experimental value compared to simulated result.

Furthermore, when the other steady-state results were compared to the simulation results, variation in the percent differences existed. The difference of effective thermal resistance was found to be $1.1 \%$ and $0.1 \%$ for Tests 1 and 2 , respectively. However, the difference for Test 4 was found to be about $7.9 \%$. The Test 4 wall design utilized a layer of VIP without XPS spacers between the VIPs to create the greatest effective thermal resistance. However, applying these to the simulation for a full-scale wall assembly, XPS was required to fill the gaps caused by the geometry of the VIPs. The geometry caused approximately $165 \mathrm{~mm}\left(6.5^{\prime \prime}\right)$ of XPS spacers to be present at the left and right composite panel edges, and a total of $445 \mathrm{~mm}$ (17.5") of XPS at the top and bottoms of composite panels. When excluding the "XPS" section of the simulation, the thermal resistance becomes $9.25 \mathrm{~m}^{2} \mathrm{~K} / \mathrm{W}$, which reduced the percent difference to $2.0 \%$ between multi-profile simulation and steady-state experimental results. Therefore, if the geometry of the panels does not fit a standard wall construction, a large amount of XPS is required to fill in the composite panel causing a large degradation in thermal performance.

The composite panels showed that the amount of XPS, linked by the required mechanical fastening area and the size of VIPs have a serious effect on the overall thermal performance of the wall assembly. The VIPs should be sized to fit within the stud cavities for both stud spacing, but also allow for a prescribed safety area between the panels. From the installation experience in lab, a $38 \mathrm{~mm}$ (1.5") fastening strip was sufficient, however in practice it is anticipated that the fastening 
strip needs to be greater. The VIPs also needed to be smaller than $609 \mathrm{~mm}$ (24") in this study, but they composite panel could be wider. The increased width would allow multiple VIPs to be added but a nailing strip should still be added between to ensure that they are not punctured. Either way, the VIPs need to fit between the stud cavities or else an excess of XPS is required, quickly degrading the thermal performance benefits from VIPs.

\subsection{Uncertainty Analysis}

The uncertainty of the guarded hot-box evaluations using Equation 1 (p.41) and Equation 2 (p.42) are presented and discussed in the upcoming sections. An uncertainty for each evaluation approach is presented with data obtained during an experimental evaluation. Uncertainties were evaluated using a combination of the volume of data and the quality of the instruments used during testing. The statistical probabilities for a $99 \%$ confidence interval based on the amount of acquired steady-state data for the surface and interface temperatures, and heat flux measurements were used. The uncertainty of the individual instruments and data acquisition equipment was included in the analysis to determine the overall uncertainty of the results.

When the effective thermal resistance of the wall assemblies were calculated using Equation 1 (p.41), there were three measured values that affected the result: two specimen surface temperatures and energy input to the system. The surface temperatures were measured at one-minute intervals at twelve locations on the interior and exterior. The twelve values were averaged during each interval to check for steady-state conditions, and averaged over the check period, usually over multiple hours, to evaluate the effective thermal resistance. The uncertainty

of a surface temperature, $U_{\mathrm{T}_{\mathrm{i}}}$, at a location $i$ is represented by Equation 5, where $B_{\mathrm{T}}$ is the bias of the thermocouple which is $0.5^{\circ} \mathrm{C}$, and $P_{\mathrm{T}_{\mathrm{i}}}$ is the precision of the measurement which is based on 
the standard deviation and a $99 \%$ confidence level of the temperature measurements at location $i$ for the entire check period at 1 minute intervals.

$$
U_{\mathrm{T}_{\mathrm{i}}}=\sqrt{{B_{\mathrm{T}}}^{2}+{P_{\mathrm{T}}}^{2}}
$$

The uncertainty of the average surface temperature, $U_{\mathrm{T}}$ is calculated using Equation 6, where the square root of the sum of the squares of the individual uncertainties is evaluated when $N$ is the number of measurement locations.

$$
U_{\mathrm{T}}=\sqrt{\sum_{i=1}^{N}\left(\frac{U_{\mathrm{T}}}{N}\right)^{2}}
$$

The uncertainty of the exterior and interior surfaces should be evaluated using this method, and the uncertainty for Test 2 of the wall assemblies using composite panels is presented. The exterior surface only contained 11 temperature sensors because of a break in a wire that rendered the measurements at one location inaccurate. The accumulated uncertainties are shown in Table 6-2, where the effects from cycling of the refrigeration unit are shown in the uncertainties of the exterior chamber. The uncertainties on the cold surface are higher than the interior surface because of the smaller temperature variations and tighter temperature control in the metering chamber. 
Table 6-2: Uncertainties for exterior and interior surface temperature

\begin{tabular}{|c|c|c|c|c|c|c|c|c|c|c|c|c|c|}
\hline \multirow[t]{2}{*}{$\begin{array}{c}\mathrm{T}_{\text {average }} \\
\left({ }^{\circ} \mathrm{C}\right)\end{array}$} & \multicolumn{12}{|c|}{$U_{\mathrm{T}_{\mathrm{i}}}\left({ }^{\circ} \mathrm{C}\right)$} & \multirow[t]{2}{*}{$\begin{array}{r}U_{\mathrm{T}} \\
\left({ }^{\circ} \mathrm{C}\right) \\
\end{array}$} \\
\hline & 1 & 2 & 3 & 4 & 5 & 6 & 7 & 8 & 9 & 10 & 11 & 12 & \\
\hline-13.57 & 1.52 & 1.18 & 1.05 & 1.41 & 0.94 & 0.79 & 1.18 & 0.94 & 1.32 & 0.86 & 0.93 & & 1.13 \\
\hline-13.67 & 1.49 & 1.13 & 1.02 & 1.38 & 0.93 & 0.78 & 1.15 & 0.93 & 1.29 & 0.85 & 0.91 & & 1.10 \\
\hline-13.69 & 1.44 & 1.12 & 1.00 & 1.36 & 0.90 & 0.76 & 1.15 & 0.91 & 1.29 & 0.84 & 0.89 & & 1.08 \\
\hline-13.65 & 1.44 & 1.12 & 0.99 & 1.38 & 0.91 & 0.78 & 1.16 & 0.90 & 1.30 & 0.84 & 0.90 & & 1.09 \\
\hline-13.57 & 1.46 & 1.13 & 1.00 & 1.38 & 0.91 & 0.77 & 1.16 & 0.91 & 1.31 & 0.84 & 0.90 & & 1.09 \\
\hline 23.17 & 0.47 & 0.46 & 0.50 & 0.47 & 0.48 & 0.46 & 0.46 & 0.46 & 0.47 & 0.47 & 0.46 & 0.47 & 0.49 \\
\hline 23.11 & 0.46 & 0.46 & 0.48 & 0.46 & 0.47 & 0.46 & 0.46 & 0.46 & 0.46 & 0.46 & 0.46 & 0.47 & 0.48 \\
\hline 23.11 & 0.46 & 0.46 & 0.48 & 0.46 & 0.47 & 0.46 & 0.46 & 0.46 & 0.46 & 0.46 & 0.46 & 0.47 & 0.49 \\
\hline 23.12 & 0.46 & 0.46 & 0.47 & 0.46 & 0.47 & 0.46 & 0.46 & 0.46 & 0.46 & 0.46 & 0.46 & 0.47 & 0.49 \\
\hline 23.16 & 0.46 & 0.46 & 0.49 & 0.47 & 0.47 & 0.46 & 0.46 & 0.46 & 0.46 & 0.46 & 0.46 & 0.47 & 0.49 \\
\hline
\end{tabular}

The energy input from the electric resistance heaters are a summation of errors based on the nominal $0.5 \%$ of full scale accuracy and the rate of pulses per Wh. Equation 7 calculates the uncertainty of the measurement where $E$ is the measured energy input to the metering chamber in watt.hours, $a$ is the nominal accuracy of $0.5 \%$ and $b$ is the $0.375 \mathrm{Wh}$ per pulse of the system, which yield an uncertainty of $0.5 \mathrm{Wh}$ for each check for composite panel Test 2 .

$$
U_{\mathrm{E}}=a E+b
$$

To evaluate the uncertainty of the effective RSI-value of a wall assembly, Equation 8 sum of squares of the partial derivative with respect to each measured variable that combines the uncertainties from the surface temperatures and the energy input. The variable $T_{\mathrm{H}}$ is the interior surface temperature in ${ }^{\circ} \mathrm{C}, T_{\mathrm{C}}$ is the exterior surface temperature in ${ }^{\circ} \mathrm{C}, E$ is the energy input to the metering chamber in Wh, $A$ is the metering area in $\mathrm{m}^{2}, U_{\mathrm{T}_{\mathrm{H}}}$ is the uncertainty of the average interior surface temperature in ${ }^{\circ} \mathrm{C}, U_{\mathrm{T}_{\mathrm{C}}}$ is the uncertainty of the average exterior surface temperature in ${ }^{\circ} \mathrm{C}$ and $U_{\mathrm{E}}$ is the uncertainty of the energy input to the metering chamber in watt.hours. 


$$
\begin{gathered}
U_{\mathrm{R}_{\mathrm{eff}}}=\sqrt{\left(\frac{\partial R_{\mathrm{eff}}}{\partial E} U_{\mathrm{E}}\right)^{2}+\left(\frac{\partial R_{\mathrm{eff}}}{\partial T_{\mathrm{H}}} U_{T_{\mathrm{H}}}\right)^{2}+\left(\frac{\partial R_{\mathrm{eff}}}{\partial T_{\mathrm{C}}} U_{T_{\mathrm{C}}}\right)^{2}} \\
U_{\mathrm{R}_{\mathrm{eff}}}=\sqrt{\left(\frac{\left(T_{\mathrm{H}}-T_{\mathrm{C}}\right) U_{\mathrm{E}} A}{E^{2}}\right)^{2}+\left(\frac{\left.U_{\mathrm{T}_{\mathrm{H}} A}\right)^{2}+\left(\frac{U_{\mathrm{T}_{\mathrm{C}}} A}{E}\right)^{2}}{E}\right.}
\end{gathered}
$$

For Test 2, the final measurement period yielded values of $21.2^{\circ} \mathrm{C} \pm 0.3^{\circ} \mathrm{C},-13.6^{\circ} \mathrm{C} \pm 0.4^{\circ} \mathrm{C}$ and 25.9 Wh $\pm 0.5 \mathrm{Wh}$. Through Equation 6, the uncertainty of the effective RSI-value from the guarded hot-box is $9.07 \mathrm{~m}^{2} \mathrm{~K} / \mathrm{W} \pm 0.25 \mathrm{~m}^{2} \mathrm{~K} / \mathrm{W}$, or $2.7 \%$ of the overall effective thermal resistance.

The second evaluation method is with heat flux plates and temperature differences on either side. Unlike evaluating the effective thermal resistance, there are less than twelve sensors used to measure the temperature difference, however, the measurements are made at the same frequency. Therefore, following Equations 3 and 4, the uncertainty of the temperature difference can be calculated. The heat flux had a measurement accuracy of $\pm 5 \%$ and was combined with a modified version of Equation 8. Equation 9 is presented with the same variables as Equation 8, with $q^{\prime \prime}$ representing the average measured heat flux in $\mathrm{W} / \mathrm{m}^{2}$.

$$
\begin{gathered}
U_{\mathrm{R}}=\sqrt{\left(\frac{\partial R}{\partial q^{\prime \prime}} U_{q^{\prime \prime}}\right)^{2}+\left(\frac{\partial R}{\partial T_{\mathrm{H}}} U_{T_{\mathrm{H}}}\right)^{2}+\left(\frac{\partial R}{\partial T_{\mathrm{C}}} U_{T_{\mathrm{C}}}\right)^{2}} \\
U_{R}=\sqrt{\left(\frac{\left(T_{\mathrm{H}}-T_{\mathrm{C}}\right) U_{\mathrm{q}^{\prime \prime}}}{q^{\prime \prime}}\right)^{2}+\left(\frac{U_{\mathrm{T}_{\mathrm{H}}}}{q^{\prime \prime}}\right)^{2}+\left(\frac{U_{\mathrm{T}_{\mathrm{C}}}}{q^{\prime \prime}}\right)^{2}}
\end{gathered}
$$

For example, the data used to evaluate the difference between the center and edge thermal resistances of VIPs found for the center of panel the temperatures were $21.2^{\circ} \mathrm{C} \pm 0.24^{\circ} \mathrm{C}$ and $5.8^{\circ} \mathrm{C} \pm 0.43^{\circ} \mathrm{C}$ with a measured heat flux of $6.5 \mathrm{~W} / \mathrm{m}^{2} \pm 0.33 \mathrm{~W} / \mathrm{m}^{2}$. By using Equation 9 , the calculated center of VIP has an RSI-value of $2.4 \mathrm{~m}^{2} \mathrm{~K} / \mathrm{W} \pm 0.14 \mathrm{~m}^{2} \mathrm{~K} / \mathrm{W}$ or an uncertainty of $6 \%$ of the experimental RSI-value. 


\subsection{VIP Integration into the Building Envelope}

The experimental and modelling work conducted for this study confirmed that a thin, high RSI-value enclosure is attainable with the use of VIPs. Concerns about edge effects and constructability were addressed during the study. Edge effects were taken into consideration by utilizing an effective RSI-value for the VIP, as opposed to using the center of panel RSI-value. The composite insulation panel addressed the constructability concerns by adding fastening strips between VIPs while maintaining an effective RSI-value above $7.04 \mathrm{~m}^{2} \mathrm{~K} / \mathrm{W}$ for the envelope. The addition of the fastening strip was significantly important in the design. The $38 \mathrm{~mm}\left(1.5^{\prime \prime}\right)$ fastening strip allowed a sufficient area to install the exterior insulation without the risk of compromising any VIPs. In practice, it is anticipated that a large area would be needed since the difference between a laboratory and a construction site would be vastly different.

In cold climates, augmenting the building envelope's thermal performance can be extremely beneficial. Since the environment's temperature is much different from the interior, in some cases below $-30^{\circ} \mathrm{C}$, the amount of heating required to maintain the interior conditions is substantial. Addressing this energy consumption by improving the building envelope, which is the boundary between the interior and exterior, without sacrificing the interior floor area, would be valuable. If the composite insulation panels had been prefabricated, delivered to the construction site, and installed with the necessary attention, the building envelope would be above RSI $7.04 \mathrm{~m}^{2} \mathrm{~K} / \mathrm{W}$ with $51 \mathrm{~mm}$ of exterior insulation. The same performance could not be achieved using materials used for code-built homes today without creating much thicker building envelopes. The studies showed that VIPs could significantly improve the thermal performance of building envelopes and help reduce space-conditioning loads while maintaining a thickness within inches of today's code-built homes. 


\subsection{Summary}

In this chapter, a discussion was presented regarding the observations and results from both the experimental and modelling studies of building envelopes with VIPs. It was found through experimental analysis that there was a $32 \%$ change in RSI-value at the VIP edge. It was discussed that the thermal performance of an envelope with VIPs could be determined an effective RSI-value using the modelling studies. In addition to these results, the infrared images of the three VIP configurations were discussed. It was found that the temperature distribution at the VIP surface is nearly homogenous when the second layer of VIPs is offset to the center-corner configuration. A comparison of analysis methods was performed for the single and multiple profile simulations. It was found that in most cases, the single profile simulation provides an effective RSI-value. The single profile simulation was within $7.0 \%$ for all but one of the five cases. The trends and highlights from the isothermal profiles of the five cases were discussed. Finally, the uncertainties of the effective RSI-value calculated using the guarded hot-box and RSI-value from the measured heat flux data was presented. The next chapter will provide concluding remarks about the findings and discuss future work. 


\section{Chapter: Conclusions}

The following conclusions can be made based on the experimental and simulation work conducted for this study.

\subsection{Configuration Effects in Multiple VIP Layers}

It was apparent through thermal imaging and embedded sensor data found at steady-state conditions that a significant drop in thermal performance existed along the perimeter of the VIPs. Before the study, there was a lack of quantified data and results to determine the reduction in RSI-value along the face of the VIP. The quantified value of RSI of the center, corner, and seam of VIPs was found to have a change in RSI between $32 \%$ at the seam and $36 \%$ at the corner when compared to the center. Since the temperature variation along the surface was, an indicator of varying RSI, in can be concluded that the center of panel RSI is stable approximately $50 \mathrm{~mm}$ (2") from the perimeter. Along the face of the panel, approximately $90 \%$ of the area is rated as the center of panel value, and allows us to conclude that an effective RSI-value for the panel as oppose to the center of panel RSI-value should be used as a material property when simulated in heat transfer programs.

\subsection{Prefabricated Panel Feasibility}

The composite panels were found to be an effective and feasible solution to the significant constructability challenge that VIPs present in residential home construction. Addressing the challenge needs before being fully integrated into the market, and based on the experience gained through installing the composite panels before steady-state testing; the design is feasible. While the laboratory conditions would be considerably different from a prospective construction site, the encasement of VIPs, as well as the labelled fastening locations, offers a high potential of installation without VIP puncture or failure. 


\subsection{Importance of Proper VIP Dimensions}

It was observed that VIP geometry could have a dramatic impact on the overall thermal resistance of the wall design within the composite panels. Through the five wall designs, a variable amount of XPS and VIP coverages were tested at steady-state and modelled in THERM to determine the effective RSI-value. For the second set of manufactured VIPs, the VIP dimensions required more XPS than the initial set of panels because the panel geometry did not align with the wall section, meaning if you install an additional column of VIPs, the wall design will not meet the minimum required fastening area. The consequence is a significantly lower effective RSI-value since a larger area of XPS spacers compared to the other designs. It highlights the importance of properly assigning VIP dimensions during the design stage of the pre-fabricated panels.

\subsection{Single Profile Modelling Effectiveness}

When modelling the panels, using symmetry or a ratio of the coverage area of specific cross sections was used to determine the effective RSI-values for Tests 1 and 2. However, the benefits of being able to modify the representative cross-section easily and the ability to use a single cross section to measure the thermal resistance are reasons to recommend using the single profile method. In contrast to the multiple profile modelling method, it is much easier to compare the composition and coverage area of two separate wall designs using the single profile method since the geometries of the wall designs would be similar. The coverage areas of VIP and XPS and associated material coverage at stud locations can be clearly seen and compared in the cross section in single cross section method, unlike multiple cross sections method. The main difference is the single cross section method requires more information about the wall assembly, specifically height ratios of cross sections, unlike the simple dimensions that the stud sizes and material covers from mulitple cross sections show. 


\subsection{Future Work}

There remains a significant amount of research required before widespread implementation of VIPs into building envelopes can be achieved. This study investigated the heat transfer through building envelopes incorporating VIPs. An important factor in the long-term performance of VIPs within building envelopes is the moisture transfer. Through the building envelope, it is important to analyze and monitor how the moisture permeates, wets, and dries throughout. If the building envelope does not have the ability to dry out as moisture permeates, mould and rot can develop. Therefore, to reach and maintain current building standards, the moisture transfer with VIPs installed must also be analyzed.

Offsetting the second layer of VIPs showed promise by performing similar to a continuous insulation by limiting the heat flux and temperature variations. The information could prove useful in mitigating the thermal and moisture performance concerns through the building envelope, however, there may be issues in the feasibility of integrating siding or finishing the exterior of the building. Issues associated with this double layered VIP insulation system could be a lack of area to nail or fasten the building finishes to the exterior without compromising the VIPs. The exterior insulation would need to be carefully designed with areas for exterior detailing and finishing to avoid wasted or punctured VIPs. A potential option to mitigate these issues could be utilizing thinner VIPs with smaller geometries to reduce the amount of area affected by a punctured VIP, while maintaining a thin, high RSI-value building envelope. Altogether, the exterior details and the VIP arrangement have to be carefully considered and studied before integrating into a building.

An unknown factor about VIPs is their thermal performance degradation over time. The current state of knowledge shows that multiple factors, such as moisture permeation, thermal cycling, or a loss of internal vacuum, contribute to a decrease in effective RSI of VIPs over time. 
While the RSI-value is known to decrease over time, it is unknown or not well proven how the performance of the panels ages through their lifetime. The degradation is an important aspect and properties of VIPs since, after installation within the building envelope, they are expected to perform for the lifetime of the building envelope which could reach 25 years or greater.

Furthermore, optimizing the dimensions of the VIPs within the assembly and the amount of XPS required to install the composite would be a valuable tool during the design of composite panels. The amount of XPS should be minimized since the effective RSI-value of $51 \mathrm{~mm}$ of XPS as exterior insulation on $304 \mathrm{~mm}(12 ")$ and $406 \mathrm{~mm}$ (16") stud spacing was $4.35 \mathrm{~m}^{2} \mathrm{~K} / \mathrm{W}$ and $4.48 \mathrm{~m}^{2} \mathrm{~K} / \mathrm{W}$, respectively. This was nearly half of the effective RSI-value after the VIPs were introduced to the building envelope. However, a minimum amount of XPS must be present as a tolerance for mechanical fastening to the dwelling. During this study, it was found that a $38 \mathrm{~mm}$ (1.5") strip could be sufficient; however, the necessary marking of where the XPS spacers are located on the surface of the composite panel is needed mitigate the risk of puncture.

Finally, since VIP puncture or loss of vacuum seem inevitable at times, a method of non-destructive quality control and testing is required for VIPs before and after installation. Even within the laboratory setting, it is difficult to tell whether a panel contains a vacuum at times. Typically, the non-destructive method of determining whether a VIP contains a vacuum is to visually inspect the panel for puncture and check that the panel remains rigid. If the panel is rigid, it was believed to contain a vacuum. However, it was found that there were instances where the panel remained rigid and did not contain as much vacuum. These panels were installed during the single and multi-layer VIP tests as they were thought to be intact.

At the end of the steady-state measurement period, thermal images were taken and revealed that it was, in fact, the VIP was compromised. The large blue surface located center-left of Figure 
3-9 is the broken panel and indicated a failed assembly and test. Further work and the investigation are required to find a method to evaluate the VIPs other than the inaccurate, subjective methods. The current state of art indicates that some panels have sensors added inside to measure the internal pressure, but not all panels are equipped with this technology. Therefore another method of non-destructive quality control needs to be developed and implemented into the panel. 


\section{References}

[1] Natural Resources Canada, "National Energy Use Handbook: 1990 to 2011," Office of Energy Efficiency, Natural Resources Canada, Ottawa, 2014.

[2] Natural Resources Canada, "The ecoENERGY Innovation Initiative," [Online]. Available: http://www.nrcan.gc.ca/energy/funding/current-funding-programs/eii.

[3] A. Parek, Next Generation Standards for Housing Efficiency, FP Innovations, 2012.

[4] buildABILITY Corporation, "Net Zero Homes," [Online]. Available: http://www.zeroenergy.ca/.

[5] US Department of Energy, "Insulation," [Online]. Available: https://energy.gov/energysaver/insulation. [Accessed October 2016].

[6] US Department of Energy, "Insulation Materials," [Online]. Available: http://energy.gov/energysaver/types-insulation. [Accessed October 2016].

[7] US Department of Energy, "Types of Insulation".

[8] P. Mukhopadhyaya, M. Kumaran, G. Sherrer and D. van Reenen, "An Investigation on Long-Term Thermal Performance of Vacuum Insulation Panels (VIPs)," in Proceedings of the 10th International Vacuum Insulation Symposium, Ottawa, 2011.

[9] S. E. Kalnaes and B. P. Jelle, "Vacuum insulation panel products: A state-of-the-art review and future research pathways," Applied Energy, no. 116, pp. 355-375, 2014. 
[10] P. Mukhopadhyaya, D. MacLean, J. Korn, D. van Reenen and S. Melleti, "Building applications and thermal performance of vacuum inuslation panels (VIPs) in Canadian subarctic climate," Energy and Buildings, vol. 85, pp. 672-680, 2014.

[11] K. Cho, Y. Hong and J. Seo, "Assessment of the economic performance of vacuum insulation panels for housing projects," Buildings and Energy, vol. 70, pp. 45-51, 2014.

[12] Natural Resources Canada, "The R-2000 Standard," Natural Resources Canada, 0412 2015. [Online]. Available: http://www.nrcan.gc.ca/energy/efficiency/housing/newhomes/5087.

[13] Canadian Passive House Institute, "Canadian Passive House Institute," 2014. [Online]. Available: http://www.passivehouse.ca/.

[14] Canada Green Building Council, "Leed Canada Rating Systems," 2014. [Online]. Available: http://www.cagbc.org/CAGBC/LEED/CommercGreenBuild/RatingSystems/CAGBC/Pro grams/LEED/CommercialInstitutional/RatingsSystems/LEED_Canada_Rating_S.aspx?hk ey=5490b62b-b10f-45b7-9c41-2b5a299655b8.

[15] S. Brunner, T. Stahl and K. Ghazi Wakili, "An example of deteriorated vacuum insulation panels in a buildings facade," Energy and Buildings, no. 54, pp. 278-282, 2012.

[16] S. Brunner, K. Ghazi Wakili, T. Stahl and B. Binder, "Vacuum insulation panels for building applications - Continuous challenges and developments," Energy and Buildings, vol. 85, pp. 592-596, 2014. 
[17] R. Baetens, B. P. Jelle, J. V. Thue, M. J. Tenpierik, S. Grynning, S. Uvslokk and A. Gustavsen, "Vacuum insulation panels for building applications: A review and beyond," Energy and Buildings, no. 42, pp. 147-172, 2010.

[18] A. Berge, B. Adl-Zarrabi and C.-E. Hagentoft, "Assessing the Thermal Performance of District Heating Twin Pipes with Vacuum Insulation Panels," Energy Procedia, vol. 78, pp. 382-387, 2015.

[19] P. Mukhopadhyaya, K. Kumaran, F. Ping and N. Normandin, "Use of vacuum insulation panel in building envelope construction: advantages and challenges," in 13 th Canadian Conference on Building Science and Technology, Winnipeg, 2011.

[20] A. Parekh and C. Mattock, "Incorporation of Vacuum Insulation Panels in a Wood Frame Net Zero Energy Home," International Vacuum Insulation Symposium, no. 10, pp. 46-50, 2011.

[21] "THERM," Lawrence Berkeley National Laboratory (LBNL), 10 March 2015. [Online]. Available: http://windows.lbl.gov/software/therm/therm.html.

[22] Fraunhofer IBP, "WUFI," [Online]. Available: https://wufi.de/en/.

[23] M. Rossi and V. Rocco, "Application of VIP: Evaluation of Winter and Summer Energy Performance of External Walls with VIP in Buildings located in Middle Latitudes," in International Vacuum Insulation Symposium, Ottawa, 2011.

[24] US Department of Energy, "Energy Plus," [Online]. Available: https://energyplus.net/.

[25] T. Voellinger, A. Bassi and M. Heitel, "Facilitating the incorporation of VIP into precast concrete sandwich panels," Energy and Buildings, no. 85, pp. 666-671, 2014. 
[26] G. vom Boegel, K.-H. Trieu, M. Goertz and A. Grabmaier, "Wireless Integrated Pressure Sensor for Quality Control of Vacuum Insulation Panel," in International Vacuum Insulation Symposium, Ottawa, 2011.

[27] F. Asdrubali and G. Baldinelli, "Thermal transmittance measurements with the hot box method: Calibration, experimental procedures and uncertainty analyses of three different approaches," Energy and Buildings, vol. 43, no. 7, pp. 1618-1626, 2011.

[28] "C1363-11 Standard Test Method for Thermal Performance of Building Materials and Envelop Assesmblies by Means of a Hot Box Apparatus," ASTM International, 2014.

[29] International Organization for Standardization, "Thermal insulation -- Determination of steady-state thermal transmission properties -- Calibrated and guarded hot box," Geneva, 2013.

[30] Gosudarstvennye Standarty State Standary, "Methods of Determination of Thermal Resistance".

[31] J. Fricke, U. Heinemann and H. Ebert, "Vacuum insulation panels -- From research to market," Vacuum, vol. 82, no. 7, pp. 680-690, 2008.

[32] J. Fricke, H. Schwab and U. Heinemann, "Vacuum insulation panels -- Exciting thermal properties and most challenging applications," International Journal of Thermophysics, vol. 27, no. 4, pp. 1123-1139, 2006.

[33] H. Schwab, C. Strak, W. Johannes, H.-P. Ebert and J. Fricke, "Thermal Bridges in Vacuum-insulated Building Facades," Journal of Building Physics, vol. 28, no. 4, pp. 345$355,2005$. 
[34] F. Bianchi, A. L. Pisello, G. Baldinelli and F. Asdrubali, "Infrared Thermography Assessment of Thermal Bridges in Building Envelope: Experimental Validation in a Test Room Setup," Sustainability, vol. 6, pp. 7107-7120, 2014.

[35] L. L. S. Zalewski and D. B. K. Rousse, "Experimental and numerical characterization of thermal bridges in prefabricated building walls," Energy Conversion and Management, no. 51, pp. 2869-2877, 2010.

[36] PHYSIBEL, "TRISCO," [Online]. Available: http://www.physibel.be/v0n2tr.htm.

[37] F. E. Boafo, J.-G. Ahn, J.-T. Kim and J.-H. Kim, "Computing thermal bridge of VIP in building retrofits using DesignBuilder," Energy Procedia, vol. 78, pp. 400-405, 2015.

[38] "DesignBuilder Software," [Online]. Available: www.designbuilder.co.uk.

[39] K. Martin, A. Campos-Celador, C. Escudero, I. Gomez and J. Sala, "Analysis of a Thermal Bridge in a Guarded Hot Box Testing Facility," University of Basque Country, Leino.

[40] K. Martin, C. E. A. Escuder, I. Flores and J. Sala, "Equivalent wall method for dynamic characterisation of thermal bridges," Energy and Buildings, no. 55, pp. 704-714, 2012.

[41] R. Baetens, B. Jelle and A. Gustavsen, "Aerogel insulation for building applications: A state-of-the-art review," Energy and Buildings, vol. 43, no. 4, pp. 761-769, 2011.

[42] A. Berge, C.-E. Hagentoft, P. Wahlgren and B. Adl-Zarrabi, "Effect from a Variable UValue in Adaptive Building Components with Controlled Internal Air Pressure," in 6th International Building Physics Conference, Italy, 2015. 
[43] C. Baldwin, "Design and Construction of an Experimental Apparatus to Assess the Performance of a Solar Absorption Chiller with Integrated Thermal Storage," 2013.

[44] Omega, "Revised Thermcouple Reference Tables - Type T Reference Tables," [Online]. Available: http://www.omega/ca/temperature/Z/pdf/z207.pdf..

[45] "Vacuum Insulation: Vacuum Insulation Panels," Panasonic, [Online]. Available: https://na.industrial.panasonic.com. [Accessed 2803 2017].

[46] R. A. Ludwig, J. Flaherty, F. Guerinoni, P. L. Baehmann and M. S. Shephard, "Adaptive Solutions of the Euler equations using finit quadtree and octree grids," Computers and Structures, vol. 30, no. 1-2, pp. 327-336, 1988.

[47] M. Schiedel, C. A. Cruickshank and C. Baldwin, "In-Situ Experimental Validation of THERM Finite Element Analysis for a High R-Value Using Vacuum Insulation Panels," in Proceedings of the ASME 2013 7th International Conference on Energy Sustainability, Minneapolis, 2013. 


\section{Appendix 1 - Instrumentation Plans for Composite Panels in Guarded Hot-box}

The appendix contained the plan view of the wall assemblies testing with composite panels with encased VIPs. The view contains the $38 \mathrm{~mm}$ by $89 \mathrm{~mm}$ (2" by 4") wood studs with the spacing dimensioned. The VIPs are included in the view by the dashed lines with the associated dimensions of VIPs and spacing between VIPs. Finally, the thermocouple instrumentation locations for the four unique cross sections are denoted by the 1 to 4 in red font and heat flux locations labelled with the "HF". All dimensions are labelled in inches.

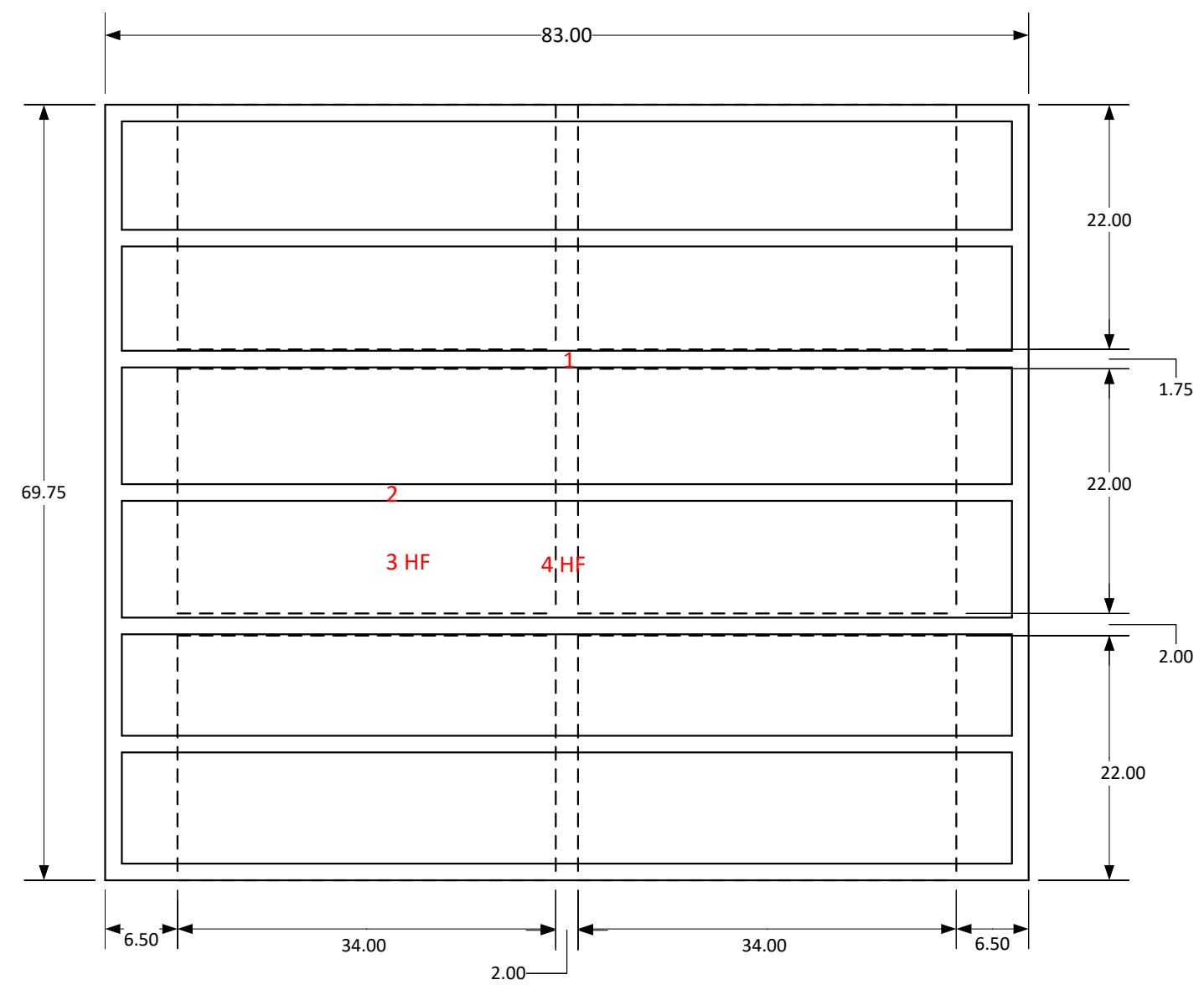

Figure A-1: Section view of Test 1 wall assembly with composite panels 


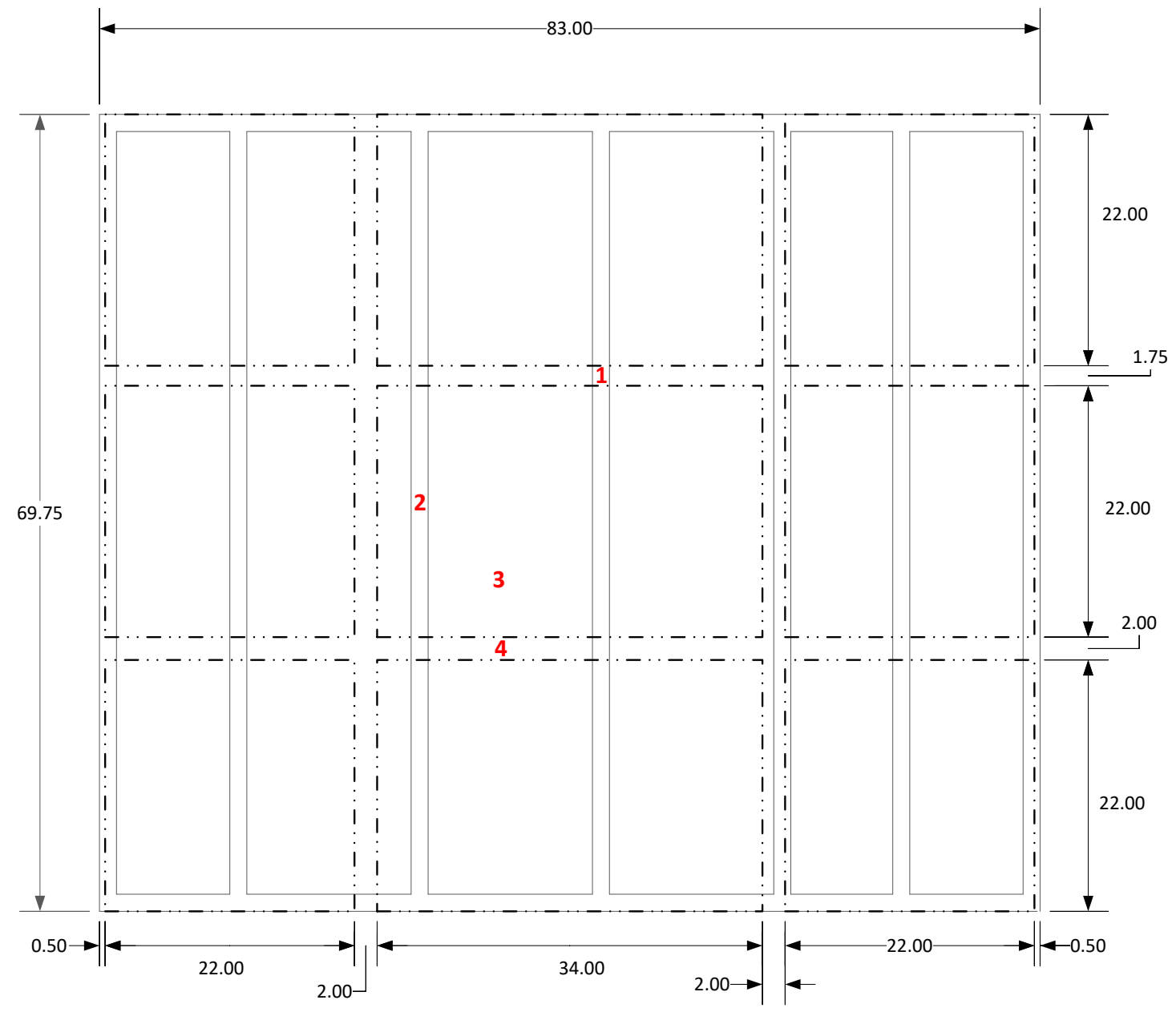

Figure A-2: Section view of Test 2 wall assembly with composite panels 


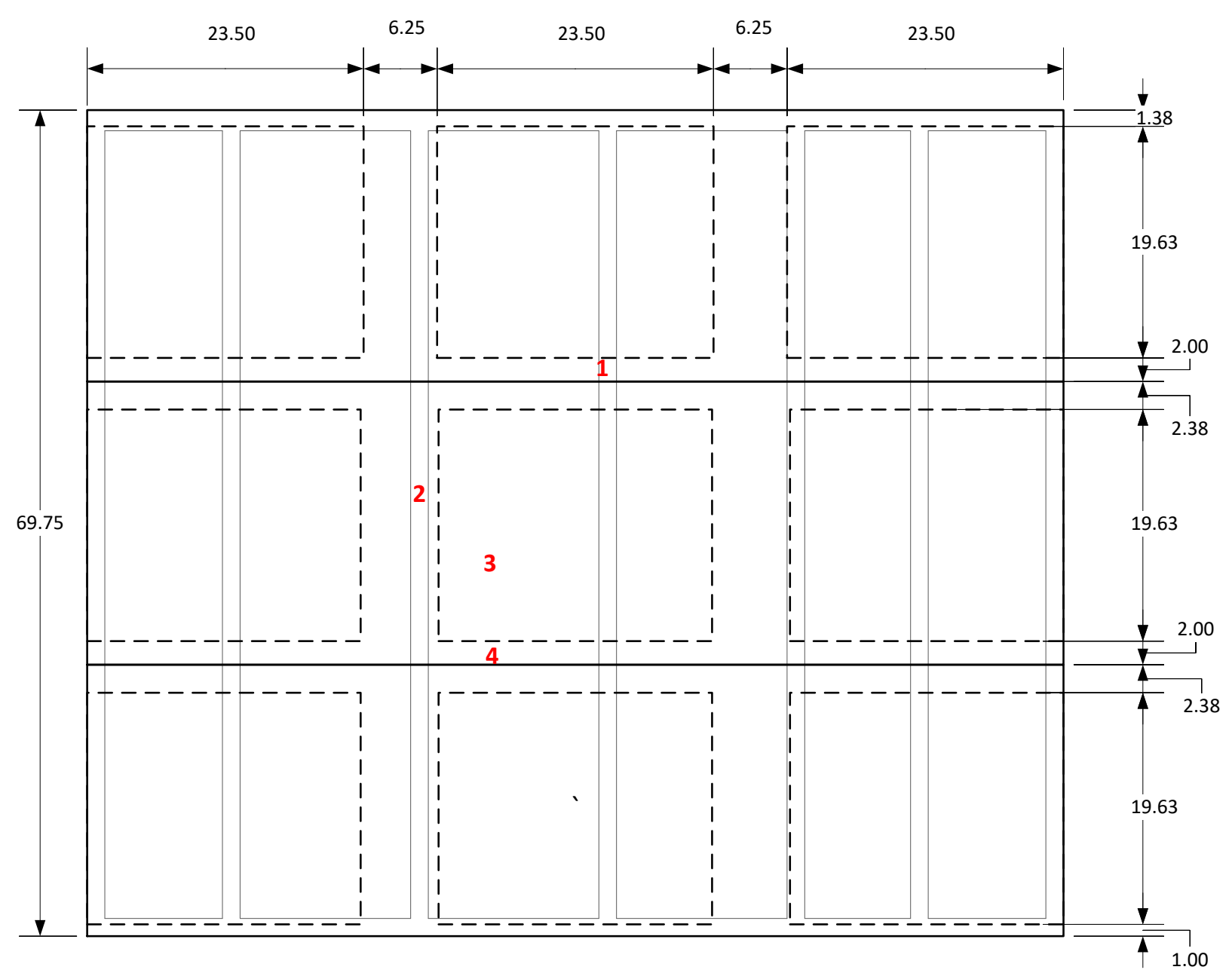

Figure A-3: Section view of Test 3 wall assembly with composite panels 


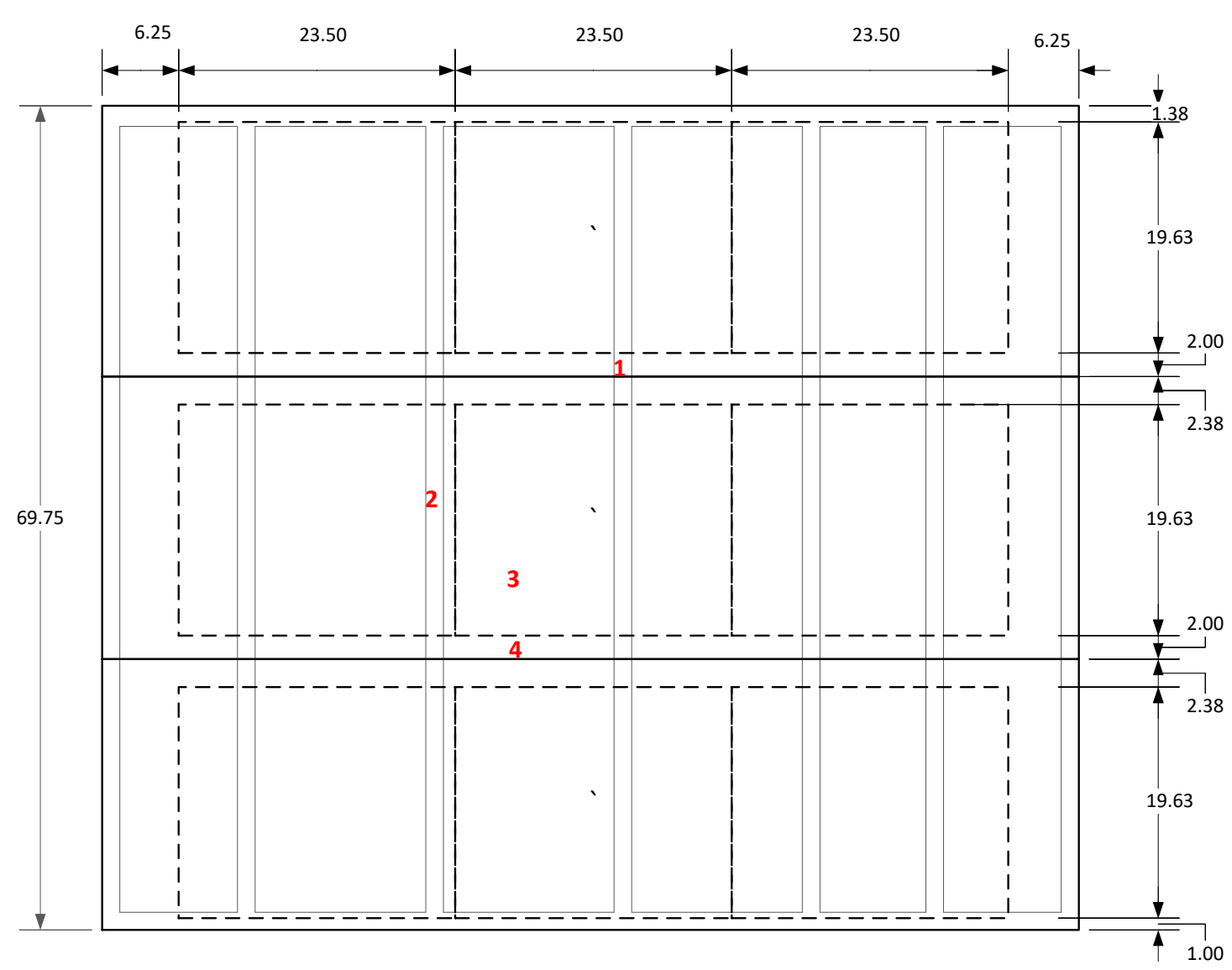

Figure A-4: Section view of Test 4 wall assembly using composite panels 


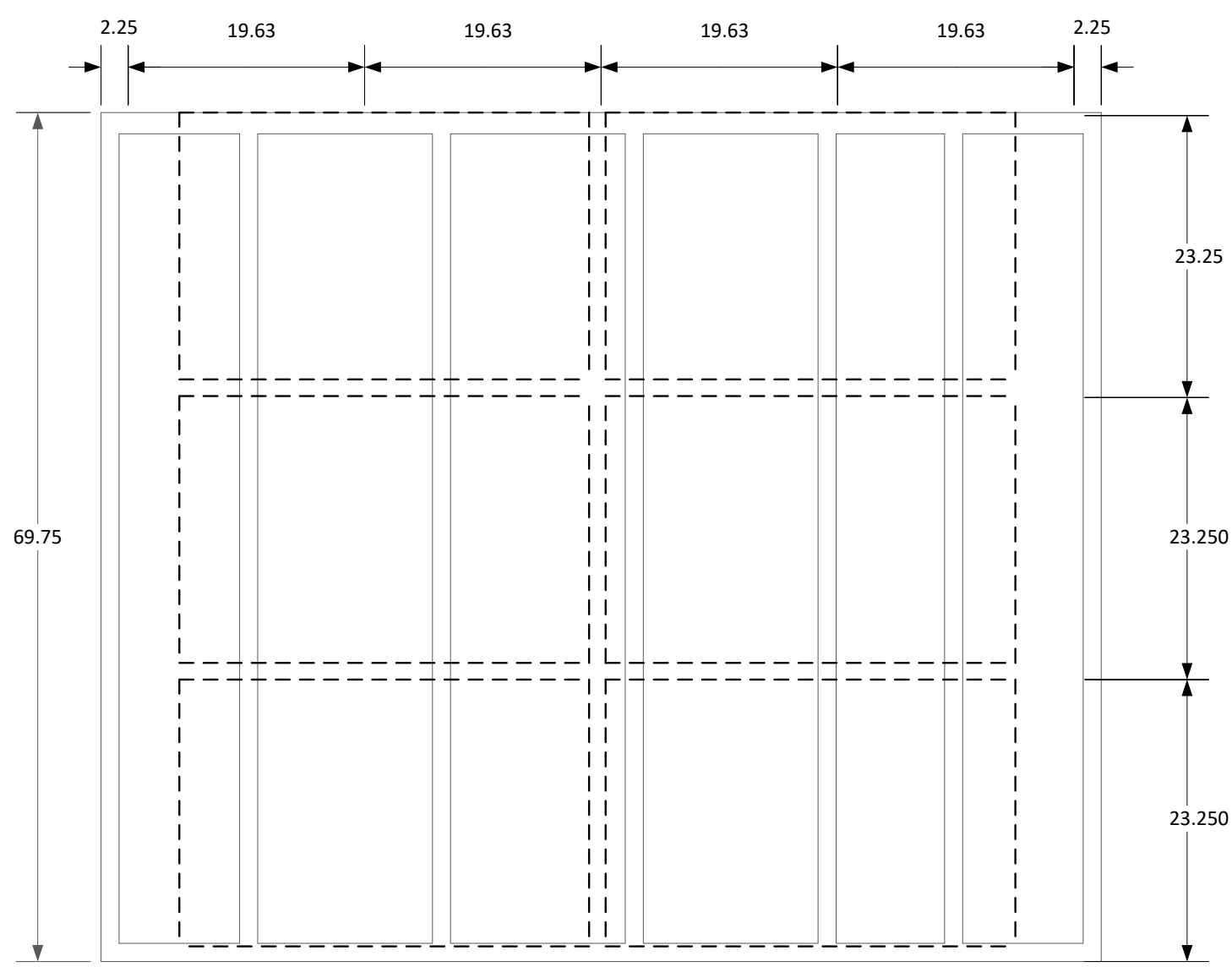

Figure A-5: Section view of Test 5 wall assembly using composite panels 